\title{
Effective field theory for non-relativistic hydrodynamics
}

\author{
Akash Jain \\ Department of Physics \& Astronomy, University of Victoria, \\ PO Box 1700 STN CSC, Victoria, BC, V8W 2Y2, Canada \\ E-mail: ajain@uvic.ca
}

AbStRact: We write down a Schwinger-Keldysh effective field theory for non-relativistic (Galilean) hydrodynamics. We use the null background construction to covariantly couple Galilean field theories to a set of background sources. In this language, Galilean hydrodynamics gets recast as relativistic hydrodynamics formulated on a one dimension higher spacetime admitting a null Killing vector. This allows us to import the existing field theoretic techniques for relativistic hydrodynamics into the Galilean setting, with minor modifications to include the additional background vector field. We use this formulation to work out an interacting field theory describing stochastic fluctuations of energy, momentum, and density modes around thermal equilibrium. We also present a translation of our results to the more conventional Newton-Cartan language, and discuss how the same can be derived via a non-relativistic limit of the effective field theory for relativistic hydrodynamics.

Keywords: Effective Field Theories, Space-Time Symmetries, Quantum Dissipative Systems, Stochastic Processes

ARXIV EPRINT: 2008.03994 


\section{Contents}

1 Introduction and overview 1

$\begin{array}{lll}2 & \text { Review of classical Galilean hydrodynamics } & 7\end{array}$

2.1 Dissipative hydrodynamics on flat background 7

$\begin{array}{lll}2.1 .1 & \text { Conservation equations } & 7\end{array}$

$\begin{array}{llr}2.1 .2 & \text { Constitutive relations } & 9\end{array}$

$\begin{array}{lll}2.2 & \text { Coupling to sources } & 11\end{array}$

$\begin{array}{lll}2.2 .1 & \text { Null backgrounds } & 11\end{array}$

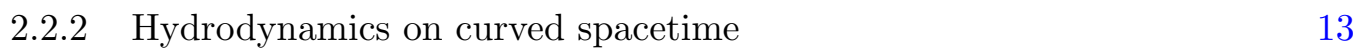

2.3 Frame transformations and thermodynamic frame 14

$\begin{array}{ll}2.4 \text { Generalities and adiabaticity equation } & 16\end{array}$

3 Effective field theory for Galilean hydrodynamics 18

$\begin{array}{ll}3.1 \text { Fluid worldvolume formulation } & 19\end{array}$

$\begin{array}{ll}3.2 & \text { Physical spacetime formulation } \\ 3.3 & \text { Sching-Keldysh }\end{array}$

3.3 Schwinger-Keldysh generating functional 24

3.4 Explicit effective action and emergent second law 26

$\begin{array}{ll}3.5 & \text { Field redefinitions and frame transformations }\end{array}$

4 Effective action for one-derivative Galilean fluids $\quad 30$

$\begin{array}{lll}4.1 & \text { Non-linear effective action } & 30\end{array}$

4.2 Linearised stochastic fluctuations $\quad 32$

4.2.1 Perturbative expansion in fluctuations 32

4.2.2 Free theory 34

$\begin{array}{lll}4.2 .3 & \text { Interactions } & 35\end{array}$

$\begin{array}{lll}4.3 & \text { Incompressible and diffusion limits } & 37\end{array}$

5 Coupling to Newton-Cartan sources $\quad \mathbf{3 8}$

5.1 Classical hydrodynamics with Newton-Cartan sources 38

$\begin{array}{lll}\text { 5.1.1 Newton-Cartan structure } & 39\end{array}$

5.1.2 Coupling to Galilean field theories 40

5.1.3 Hydrodynamics on curved spacetime $\quad 42$

5.2 Schwinger-Keldysh effective field theory 44

5.2.1 Effective field theory on fluid worldvolume 45

5.2.2 Effective field theory on physical spacetime 47

5.2.3 Schwinger-Keldysh constraints and explicit effective action $\quad 50$

6 Outlook 53

A Second law constraints in Galilean hydrodynamics $\quad 55$ 
B Effective field theory via non-relativistic limit

$\begin{array}{ll}\text { B.1 Relativistic hydrodynamics } & 56\end{array}$

$\begin{array}{ll}\text { B.1.1 Classical hydrodynamics } & 56\end{array}$

B.1.2 Schwinger-Keldysh effective field theory 58

$\begin{array}{lll}\text { B.2 Non-relativistic limit } & 60\end{array}$

B.2.1 Newton-Cartan sources via non-relativistic limit 60

$\begin{array}{lll}\text { B.2.2 Non-relativistic limit of hydrodynamics } & 61\end{array}$

\section{Introduction and overview}

Hydrodynamics is an effective framework that aims to describe low-energy dynamics of many body systems near thermal equilibrium. Classically, hydrodynamics is posed as a set of conservation equations associated with the global symmetries that the system enjoys. The underlying rationale is that non-conserved quantities are expected to decay and thermalise at much smaller distance and time scales compared to conserved quantities, which need to be transported out to infinity to thermalise. Hence, one expects that if one waits long enough and is only interested in macroscopic phenomenon, the low-energy behaviour of a thermal system would be entirely governed by the dynamics of its conserved operators. However, this expectation is only true up to a leading approximation. As we start to take into account interactions between various hydrodynamic modes, the inevitable interaction between hydrodynamic and the ignored high-energy degrees of freedom also starts to play an important role. A concrete realisation of this phenomenon are the so-called long-time tails $[1-3]$, which signal the breakdown of the hydrodynamic derivative expansion at late times. To account for such effects, one needs to pass to the more sophisticated formalism of stochastic hydrodynamics (see e.g. [4]). Here, the collective high-energy excitations are modelled by random small-scale noise sources in the hydrodynamic equations [5-9], followed by statistical averaging over all possible noise configurations. The noisy interactions are chosen in a way that they reproduce the classical hydrodynamic results in the appropriate limit. This formalism is still highly phenomenological and minimalistic and does not account for the most generic structure of stochastic interactions nor quantum fluctuations of the hydrodynamic modes themselves.

Ideally, one would like to not base the hydrodynamic framework on a set of conservation equations, but rather write down a bona fide effective field theory (EFT) starting from certain fundamental degrees of freedom and symmetries. This would systematically account for the high-energy stochastic interactions without having to introduce them by hand. Thanks to a series of collaborated efforts over the last decade, there now exists a complete EFT description for stochastic hydrodynamics based on the Schwinger-Keldysh (SK) formalism of thermal field theories [10-20]; see [21] for a review. One of the major achievements of the new framework is an understanding of the second law of thermodynamics as a macroscopic manifestation of the microscopic KMS (Kubo-Martin-Schwinger) condition. The KMS condition also ensures that the hydrodynamic correlators predicted by the 
effective theory satisfy non-linear fluctuation-dissipation theorems [22]. So far, such field theoretic tools have been developed for a handful of low-energy condensed matter systems including relativistic fluids and superfluids [12, 13], and Abelian and non-Abelian diffusive fluctuations $[23,24]$. See also, [25-27] for similar tools in non-dissipative relativistic solids, supersolids, liquid crystals, relativistic magnetohydrodynamics, and force-free electromagnetism. Nonetheless, an effective field theory approach for non-relativistic (Galilean) hydrodynamics is still largely missing. This is an obvious limitation because most physical situations around us where such a theory could be useful are non-relativistic. The goal of this paper is to remedy this situation and write down a complete Schwinger-Keldysh effective field theory for dissipative Galilean hydrodynamics.

A primary ingredient in the EFT framework are a set of background fields coupled to various conserved operators that make up the spectrum of hydrodynamics. These act as sources in the associated field theory generating functional and can be used to obtain correlation functions of the hydrodynamic operators. For example, in a relativistic setting it is widely known that the energy momentum tensor $T^{\mu \nu}$ and charge (particle number) current $J^{\mu}$ can be coupled to a background spacetime metric $g_{\mu \nu}$ and gauge field $A_{\mu}$ respectively. The situation is slightly more tricky in the Galilean case. The hydrodynamic observables are the mass (particle number) density $\rho^{t}$, mass (particle number) flux/momentum density $\rho^{i}$, energy density $\epsilon^{t}$, energy flux $\epsilon^{i}$, and stress tensor $\tau^{i j}$. These need to be coupled to the so-called Newton-Cartan (NC) structure: mass gauge field $\left(A_{t}, A_{i}\right)$, clock form $\left(n_{t}, n_{i}\right)$, and spatial metric $h_{i j}$ respectively, that describes a curved non-relativistic spacetime [28-45]; see [30] for a review. ${ }^{1}$ However, Galilean boost symmetry (also referred to as Milne boosts in the NC literature) acts on these quantities quite non-trivially and is quite tedious to implement in an effective theory written in the NC language. It is this symmetry that is responsible for setting the momentum density equal to the mass flux.

Fortunately, there is another manifestly covariant formulation for coupling Galilean field theories to background sources, using the so-called null backgrounds [46]. The idea is that by introducing a fiducial null coordinate $x^{-}$, Galilean background sources can be arranged into a one-dimensional higher relativistic metric $g_{\mathrm{MN}} \mathrm{d} x^{\mathrm{M}} \mathrm{d} x^{\mathrm{N}}=$ $-2\left(\mathrm{~d} x^{-}-A_{t} \mathrm{~d} t-A_{i} \mathrm{~d} x^{i}\right)\left(n_{t} \mathrm{~d} t+n_{i} \mathrm{~d} x^{i}\right)+h_{i j} \mathrm{~d} x^{i} \mathrm{~d} x^{j}$. Analogously, the Galilean hydrodynamic observables also arrange themselves neatly into a one-dimensional higher relativistic energy-momentum tensor $T^{\mathrm{MN}}$ coupled to $g_{\mathrm{MN}}$. Galilean symmetries, including boosts, act on these quantities naturally as one-dimensional higher Poincaré transformations that leave the null vector $V^{\mathrm{M}} \partial_{\mathrm{M}}=\partial_{-}$invariant. Posed in these terms, Galilean physics gets dressed as one-dimensional higher relativistic physics with a null Killing vector $V^{\mathrm{M}}$. The NC results can always be obtained from here by reducing the theory over the fiducial null coordinate, formally known as null reduction [47-49]. Not only does this formalism makes all the symmetries of the Galilean theory manifest, it also allows one to directly import a plethora of well-developed relativistic results directly into the Galilean setting with only minor modifications to accommodate the null Killing vector; see e.g. [46, 50-59]. Along

\footnotetext{
${ }^{1}$ Here we have chosen the Newton-Cartan Galilean frame velocity $v^{i}=0$. A more formal discussion on Newton-Cartan backgrounds is given in section 5.1.
} 
the same lines, for our current goal of interest, we can use the null background framework to write down an effective field theory for Galilean hydrodynamics using the techniques already developed for relativistic hydrodynamics.

For the benefit of the interested readers who might not want to get their hands dirty with all the technicalities, we summarise the main results of the paper here in as nontechnical terms as we can manage. The fundamental degrees of freedom in the EFT for hydrodynamics, relativistic or Galilean, are the comoving coordinates of the fluid elements $\sigma^{i}(x)$, comoving time coordinate $\sigma^{\tau}(x)$, mass/particle number phase $\varphi_{r}(x)$. These are accompanied by associated stochastic noise fields $X_{a}^{i}(x), X_{a}^{t}(x)$, and $\varphi_{a}(x)$ capturing our statistical ignorance for the respective quantities. Here $\left(x^{\mu}\right)=\left(t, x^{i}\right)$ collectively denotes physical time and space coordinates. The theory is coupled to two copies of background sources in spirit with the SK formalism; for the Galilean case these are the "difference" sources $A_{a \mu}(x), n_{a \mu}(x)$, and $h_{a \mu \nu}(x)$ (with $h_{a \mu t}(x)=0$ ) that probe the physical mass density-flux $\rho_{r}^{\mu}$, energy density-flux $\epsilon_{r}^{\mu}$, and stress tensor $\tau_{r}^{\mu \nu}$ (normalised as $\tau_{r}^{\mu \nu} n_{r \nu}=0$ ) operators respectively, while the "average" sources $A_{r \mu}(x), n_{r \mu}(x)$, and $h_{r \mu \nu}(x)\left(\right.$ with $\left.h_{r \mu t}(x)=0\right)$ probe the respective stochastic noise operators $\rho_{a}^{\mu}, \epsilon_{a}^{\mu}$, and $\tau_{a}^{\mu \nu}$ (normalised as $\tau_{a}^{\mu \nu} n_{r \nu}=0$ ). This " $r / a$ " usage for background fields and associated operators is standard in the SK literature; see e.g. [60]. The "classical" hydrodynamic fields are defined in terms of these as

$$
\begin{array}{ll}
\text { Velocity : } \quad u^{\mu}=\frac{\beta^{\mu}}{n_{r \mu} \beta^{\mu}}, & \text { Temperature : } \quad k_{\mathrm{B}} T=\frac{1}{n_{r \mu} \beta^{\mu}}, \\
\text { Mass chemical potential : } & \mu=\frac{\Lambda_{\beta}+\beta^{\nu} A_{r \nu}}{n_{r \mu} \beta^{\mu}}+\frac{1}{2} \frac{h_{r \nu \rho} \beta^{\nu} \beta^{\rho}}{\left(n_{r \mu} \beta^{\mu}\right)^{2}}
\end{array}
$$

where $\beta^{\mu}(x)=\beta_{0} \partial x^{\mu}(\sigma(x)) / \partial \sigma^{\tau}(x)$ is called the thermal vector and $\Lambda_{\beta}(x)=\beta_{0} \mu_{0}+$ $\beta^{\mu}(x) \partial_{\mu} \varphi_{r}(x)$ the chemical shift field, with $x^{\mu}(\sigma)$ being the inverse of $\sigma^{\alpha}(x)$. Furthermore, $\beta_{0}=\left(k_{\mathrm{B}} T_{0}\right)^{-1}$ and $\mu_{0}$ are the constant inverse temperature and chemical potential of the global thermal state respectively. Note that the fluid velocity $u^{\mu}$ is normalised as $u^{\mu} n_{r \mu}=1$ and only has $d$ independent components in $d$ spatial dimensions. It is also convenient to introduce a Galilean frame velocity $v^{\mu}=\delta_{t}^{\mu} / n_{r t}$, obeying $v^{\mu} n_{r \mu}=1$. We can define the inverse spatial metric as $h_{r}^{\mu \nu}$ with components $h_{r}^{t t}=h_{r}^{i j} n_{r i} n_{r j} / n_{r t}^{2}, h_{r}^{t i}=-h_{r}^{i j} n_{r j} / n_{r t}$, and $h_{r}^{i j}=\left(h_{r}^{-1}\right)^{i j}$, satisfying $h_{r}^{\mu \nu} n_{r \mu}=0$ and $h_{r}^{\mu \lambda} h_{\lambda \nu}+v^{\mu} n_{r \nu}=\delta_{\nu}^{\mu}$. Note that $h_{r}^{\mu \nu}$ is not the inverse of $h_{r \mu \nu}$.

At ideal order, a Galilean fluid is characterised by the grand-canonical equation of state, i.e. its thermodynamic pressure $p(T, \mu)$ expressed as a function of $T$ and $\mu$. Its derivatives define the thermodynamic densities: mass density $\rho(T, \mu)$, internal energy density $\varepsilon(T, \mu)$, and entropy density $s(T, \mu)$ via the Gibbs-Duhem relation $\mathrm{d} p=s \mathrm{~d} T+\rho \mathrm{d} \mu$ and Euler relation $\varepsilon+p=T s+\mu \rho$. Note that these two imply the local first law of thermodynamics $\mathrm{d} \varepsilon=T \mathrm{~d} s+\mu \mathrm{d} \rho$. One-derivative dissipative corrections to ideal hydrodynamics are parametrised by three non-negative transport coefficients: shear viscosity $\eta(T, \mu)$, bulk viscosity $\zeta(T, \mu)$, and thermal conductivity $\kappa(T, \mu)$. For a parity-violating fluid, there are further non-dissipative corrections that can be found later in the main text. In $d$ spatial 
dimensions, the fluid is described by the effective action

$$
\begin{aligned}
S= & \int \mathrm{d} t \mathrm{~d}^{d} x n_{r t} \sqrt{\operatorname{det} h_{r}}\left[\rho u^{\mu}\left(A_{a \mu}+\partial_{\mu} \varphi_{a}+£_{X_{a}} A_{r \mu}\right)\right. \\
& -\left(\left(\varepsilon+p+\frac{1}{2} \rho h_{r \rho \sigma} u^{\rho} u^{\sigma}\right) u^{\mu}-p v^{\mu}\right)\left(n_{a \mu}+£_{X_{a}} n_{r \mu}\right)+\frac{1}{2}\left(\rho u^{\mu} u^{\nu}+p h_{r}^{\mu \nu}\right)\left(h_{a \mu \nu}+£_{X_{a}} h_{r \mu \nu}\right) \\
& +\frac{i k_{\mathrm{B}} T}{4}\left(2 \eta h_{r}^{\mu(\rho} h_{r}^{\sigma) \nu}+\left(\zeta-\frac{2}{d} \eta\right) h_{r}^{\mu \nu} h_{r}^{\rho \sigma}\right)\left(h_{a \mu \nu}+£_{X_{a}} h_{r \mu \nu}-2\left(n_{a(\mu}+£_{X_{a}} n_{r(\mu}\right) h_{r \nu) \lambda} u^{\lambda}\right) \\
& \quad\left(h_{a \rho \sigma}+£_{\left(X_{a}+i \beta\right)} h_{r \rho \sigma}-2\left(n_{a(\mu}+£_{\left(X_{a}+i \beta\right)} n_{r(\mu}\right) h_{r \nu) \lambda} u^{\lambda}\right) \\
& \left.+i k_{\mathrm{B}} T^{2} \kappa h_{r}^{\mu \nu}\left(n_{a \mu}+£_{X_{a}} n_{r \mu}\right)\left(n_{a \nu}+£_{\left(X_{a}+i \beta\right)} n_{r \nu}\right)\right] .
\end{aligned}
$$

Here $£_{X_{a}}$ denotes a Lie derivative along $X_{a}^{\mu}$, while $£_{\left(X_{a}+i \beta\right)}$ along $X_{a}^{\mu}+i u^{\mu} / T$. Varying with respect to the difference sources leads to the well-known constitutive relations of onederivative Galilean hydrodynamics, modified with stochastic noise contributions. Upon setting the sources to trivial: $A_{a \mu}=n_{a \mu}=h_{a \mu \nu}=0, A_{r \mu}=0, n_{r \mu}=\delta_{\mu}^{t}$, and $h_{r \mu \nu}=\delta_{\mu}^{i} \delta_{\nu}^{j} \delta_{i j}$, these are reduced to

$$
\begin{aligned}
\rho_{r}^{t} & =\rho, \quad \rho_{r}^{i}=\rho u^{i}, \quad \epsilon_{r}^{t}=\varepsilon+\frac{1}{2} \rho \vec{u}^{2} \\
\epsilon_{r}^{i} & =\left(\varepsilon+p+\frac{1}{2} \rho \vec{u}^{2}\right) u^{i}-2 \eta u_{j} \partial^{(i} u^{j)}-\left(\zeta-\frac{2}{d} \eta\right) u^{i} \partial_{k} u^{k}-\kappa \partial^{i} T+i \epsilon_{\text {noise }}^{i} \\
\tau_{r}^{i j} & =\rho u^{i} u^{j}+p \delta^{i j}-2 \eta \partial^{(i} u^{j)}-\zeta \delta^{i j} \partial_{k} u^{k}+i \tau_{\text {noise }}^{i j}
\end{aligned}
$$

where $\epsilon_{\text {noise }}^{i}$ and $\tau_{\text {noise }}^{i j}$ are the stochastic noise contributions given by

$$
\begin{aligned}
& \epsilon_{\text {noise }}^{i}=4 k_{\mathrm{B}} T \eta u_{j}\left(\partial^{(i} X_{a}^{j)}-u^{(i} \partial^{j)} X_{a}^{t}\right)+2 k_{\mathrm{B}} T\left(\zeta-\frac{2}{d} \eta\right) u^{i}\left(\partial_{k} X_{a}^{k}-u^{k} \partial_{k} X_{a}^{t}\right)+2 k_{\mathrm{B}} T^{2} \kappa \partial^{i} X_{a}^{t}, \\
& \tau_{\text {noise }}^{i j}=4 k_{\mathrm{B}} T \eta\left(\partial^{(i} X_{a}^{j)}-u^{(i} \partial^{j)} X_{a}^{t}\right)+2 k_{\mathrm{B}} T\left(\zeta-\frac{2}{d} \eta\right) \delta^{i j}\left(\partial_{k} X_{a}^{k}-u^{k} \partial_{k} X_{a}^{t}\right)+2 k_{\mathrm{B}} T^{2} \kappa \partial^{i} X_{a}^{t} .
\end{aligned}
$$

Varying with respect to the average sources, on the other hand, one can also obtain the partner noise operators: $\rho_{a}^{t}, \rho_{a}^{i}, \epsilon_{a}^{t}, \epsilon_{a}^{i}$, and $\tau_{a}^{i j}$. These are fairly complicated, so we do not present them here. In the absence of sources, the effective action itself turns into

$$
\begin{aligned}
S= & \int \mathrm{d} t \mathrm{~d}^{3} x\left[\rho^{t} \partial_{t} \varphi_{a}+\rho^{i} \partial_{i} \varphi_{a}-\epsilon^{t} \partial_{t} X_{a}^{t}-\epsilon^{i} \partial_{i} X_{a}^{t}+\rho^{i} \partial_{t} X_{a i}+\tau^{i j} \partial_{i} X_{a j}\right. \\
& +2 i k_{\mathrm{B}} T \eta\left(\partial_{(i} X_{a j)}-u_{(i} \partial_{j)} X_{a}^{t}\right)\left(\partial^{(i} X_{a}^{j)}-u^{(i} \partial^{j)} X_{a}^{t}\right)+i k_{\mathrm{B}} T\left(\zeta-\frac{2}{d} \eta\right)\left(\partial_{k} X_{a}^{k}-u^{k} \partial_{k} X_{a}^{t}\right)^{2} \\
& \left.+i k_{\mathrm{B}} T^{2} \kappa \partial_{i} X_{a}^{t} \partial^{i} X_{a}^{t}\right] .
\end{aligned}
$$

Here $\rho^{t}, \rho^{i}, \epsilon^{t}, \epsilon^{i}$, and $\tau^{i j}$ (without any subscript) are the classical constitutive relations, obtained from eq. (1.3) by turning off the noise contributions. One can verify that varying with respect to $\varphi_{a}, X_{a}^{t}$, and $X_{a}^{i}$ leads to the well-known conservation equations of Galilean hydrodynamics in the absence of noise: $\partial_{t} \rho^{t}+\partial_{i} \rho^{i}=0, \partial_{t} \epsilon^{t}+\partial_{i} \epsilon^{i}=0$, and $\partial_{t} \rho^{i}+\partial_{j} \tau^{i j}=0$. 
Note that the effective action has a non-zero imaginary part coupled to dissipative transport coefficients $\eta, \zeta$, and $\kappa$. This captures the fact that the effective action is dissipative.

To study the effects of stochastic fluctuations around an equilibrium configuration, we can expand eq. (1.5) order-by-order in fluctuations around the state $\sigma^{i}(x)=x^{i}, \sigma^{\tau}(x)=t$, $\varphi_{r}(x)=0$, and $X_{a}^{i}(x)=X_{a}^{t}(x)=\varphi_{a}(x)=0$. Truncating the expansion at the desired order in interactions, depending on the sensitivity required, we can set up a diagrammatic analysis for computing the hydrodynamic correlation functions. Detailed expressions for the effective action truncated at three-point interactions are given in section 4. A similar analysis was performed by the authors of [23], but restricted to just energy fluctuations, to compute non-analytic long-time tail behaviour in the energy-energy retarded two-point function. By contrast, the effective action (1.5) is suitable for describing all energy, momentum, and density fluctuations.

Given the effective action coupled to sources, we can define the SK generating functional for the EFT via the path integral $\exp W\left[\phi_{r}, \phi_{a}\right]=\int \mathcal{D} \sigma \mathcal{D} \varphi_{r} \mathcal{D} X_{a} \mathcal{D} \varphi_{a} \exp (i S)$, which is a functional of the background sources $\phi_{r, a}=\left(A_{r, a t}, A_{r, a i},-n_{r, a t},-n_{r, a i}, h_{r, a i j}\right)$. This can be used to compute the correlation functions of the hydrodynamic operators $\mathcal{O}_{r, a}=\left(\rho_{r, a}^{t}, \rho_{r, a}^{i}, \epsilon_{r, a}^{t}, \epsilon_{r, a}^{i}, \tau_{r, a}^{i j}\right)$ using the variational formulae

$$
G_{\mathcal{O}_{\alpha} \ldots}(x, \ldots)=i^{n_{a}}\left(\frac{-i \delta}{\delta \phi_{\bar{\alpha}}(x)} \ldots\right) W\left[\phi_{r}, \phi_{a}\right]
$$

where $\alpha=r, a$ and $\bar{\alpha}=a, r$ denotes its conjugate, while $n_{a}$ is the number of $a$ type fields in the correlator on the left. Following the general SK machinery (see e.g. [22]), the correlators of the type " $r a \ldots a$ " are interpreted as the fully retarded correlation functions, "a...ar" as the fully advanced, "rr...r" as the symmetric correlation functions, and various other time-ordering schemes in between. For instance, $G_{\mathcal{O}_{r} \mathcal{O}_{a}}, G_{\mathcal{O}_{a} \mathcal{O}_{r}}, G_{\mathcal{O}_{r} \mathcal{O}_{r}}$ are the retarded, advanced, and symmetric propagators of the hydrodynamic observables. The SK generating functional describing an out-of-equilibrium thermal system is expected to satisfy a number of consistency conditions. These are reflected as a set of constraints in the effective action as proposed by [12]. (1) The effective action should map to minus its complex conjugate when we flip the signs of all the " $a$ " type fields. This results in $W\left[\phi_{r}, \phi_{a}\right]=W^{*}\left[\phi_{r},-\phi_{a}\right]$ and ensures that all the correlations functions are real (in position space). (2) The action should vanish when we switch off all the " $a$ " type fields, which ensures that $W\left[\phi_{r}, \phi_{a}=0\right]=0$ and all the correlation functions of the type "aa ... $a$ " identically vanish. $^{2}$ (3) The imaginary part of the effective action should be positive semi-definite, leading to $\operatorname{Re} W\left[\phi_{r}, \phi_{a}\right] \leq 0$, which ensures that variously ordered correlation functions have the appropriate singularity structure in the momentum space. (4) Lastly,

\footnotetext{
${ }^{2}$ Originally, it was believed that one needs to introduce additional BRST ghost fields and emergent supersymmetry into the hydrodynamic setup to ensure that this condition is satisfied at all loop orders [12]. However, it is now understood that one can avoid these ghost fields altogether by employing a consistent regularisation procedure for frequency loop integrals [17].
} 
the effective action should be invariant under the discrete KMS symmetry given by ${ }^{3}$

$$
\begin{array}{lll}
\phi_{r}(x) & \rightarrow \phi_{r}(-x), & \phi_{a}(x) \rightarrow \phi_{a}(-x)-i \beta_{0} \partial_{t} \phi_{r}(-x), \\
\beta^{\mu}(x) \rightarrow \beta^{\mu}(-x), & \Lambda_{\beta}(x) \rightarrow \Lambda_{\beta}(-x), \\
X_{a}^{\mu}(x) \rightarrow-X_{a}^{\mu}(-x)-i \beta^{\mu}(-x)+i \beta_{0} \delta_{t}^{\mu}, & \varphi_{a}(x) \rightarrow-\varphi_{a}(-x)-i \Lambda_{\beta}(-x) .
\end{array}
$$

This ensures that the correlations functions (1.6) satisfy (non-linear) fluctuation-dissipation theorems [22]. These conditions are together responsible for an emergent local second law of thermodynamics and Onsager's reciprocity relations [14]. In addition to the SK constraints, the effective action for hydrodynamics has to be invariant under (5) reparametrisations of the comoving spacetime coordinates associated with the fluid elements and, of course, for Galilean hydrodynamics under (6) local Galilean transformations of the background sources, including Milne boosts. It can be checked that the effective action (1.2) obeys all these requirements. In the main text, we look at these symmetries and conditions in more detail and outline the construction of the most generic effective action for Galilean hydrodynamics allowed by the EFT framework.

The organisation of the remainder of the paper is as follows. We start section 2 with a lightening review of classical Galilean hydrodynamics and its coupling to null background sources. We introduce the local second law of thermodynamics and derive the Galilean adiabaticity equation encapsulating the second law constraints to arbitrary high orders in the derivative expansion. We also spend some time discussing the subtle issue of hydrodynamic frame transformations. Section 3 makes up the bulk of the paper. Here we give a detailed construction of the EFT framework for Galilean hydrodynamics based on symmetry principles and SK formalism of thermal field theory. Also in this section we construct the most generic effective action consistent with the framework, in small $\hbar$ limit, and discuss its connection to classical hydrodynamics. A proof of the local second law of thermodynamics, within the context of the effective theory, also appears here. Leaving formalities aside, we discuss the explicit effective action for one-derivative Galilean hydrodynamics in section 4 . We linearise this action around a fluid configuration at rest on a flat background and setup a perturbative theory for stochastic fluctuations, keeping terms up to three-point interactions. In section 5 , we translate our results to the Newton-Cartan language; the Milne boost symmetry is no longer manifest but the results can be presented without introducing an auxiliary spacetime direction. Finally, we close the paper with some discussion in section 6. The paper has two appendices. In appendix A, we give a brief derivation of the second law constraints in one-derivative Galilean hydrodynamics. In appendix B, we illustrate how the effective action for Galilean hydrodynamics arises through a large speed of light expansion of its relativistic cousin.

Factors of $\hbar, c$, and $k_{\mathrm{B}}$ have been kept explicit throughout this paper. Similarly, constant global temperature $T_{0}$ and chemical potential $\mu_{0}$ appear explicitly. While we generically work in $d$ spatial dimensions, the parity-violating results are presented only for $d=3$.

\footnotetext{
${ }^{3}$ Defining $\phi_{1,2}=\phi_{r} \pm \hbar / 2 \phi_{a}$, background field transformations in eq. (1.7) are merely the small $\hbar$ limit of the KMS transformation $\phi_{1}(x) \rightarrow \phi_{1}(-x)$ and $\phi_{2}(x) \rightarrow \phi_{2}\left(-t-i \hbar \beta_{0},-\vec{x}\right)$.
} 


\section{Review of classical Galilean hydrodynamics}

Before we pursue an EFT for Galilean hydrodynamics, let us take a step back and review the essential features of classical Galilean hydrodynamics. We initiate our discussion with Galilean symmetries and associated conservation equations and derive the constitutive relations of a dissipative Galilean fluid in section 2.1. We mainly follow the textbook approach of [61], with a few novel features derived from [31] for parity-violating fluids. We review the null fluid framework of [46] to couple Galilean hydrodynamics to background sources in section 2.2. The discussion in terms of the more widely used Newton-Cartan sources has been deferred to section 5. Subtleties regarding frame transformations in hydrodynamics have been discussed in section 2.3. Finally, in section 2.4, we outline an all-order argument to derive the Galilean hydrodynamic constitutive relations in accordance with the second law of thermodynamics using the offshell formalism [57, 62].

\subsection{Dissipative hydrodynamics on flat background}

Classical hydrodynamics is a theory of locally conserved quantities. One sets out by enumerating the complete set of Noether currents associated with any global symmetries that the system might enjoy, and expresses various "fluxes" in terms of the conserved "densities", arranged in a perturbative expansion in derivatives. For a given set of such "constitutive relations", the time evolution of the conserved densities is determined by their respective conservation equations. In Galilean (non-relativistic) hydrodynamics, the conserved densities of interest are the energy, momentum, and mass/particle number density of the fluid. The associated fluxes are the energy flux and stress tensor; mass flux is the same as momentum density due to Galilean symmetries. Up to one-derivative order, the constitutive relations are specified in terms of a thermodynamic equation of state, along with number of one-derivative transport coefficients such as viscosities and conductivity.

\subsubsection{Conservation equations}

Let us consider a field theory in $d$ spatial dimensions with coordinates $t, x^{i}$ with $i=1,2, \ldots d$. For the field theory to qualify as Galilean, the dynamical evolution must be invariant under global Galilean coordinate transformations: translations $\left(a^{i}\right)$, time-translation $\left(a^{t}\right), \mathrm{SO}(d)$ rotations $\left(\Lambda_{j}^{i}\right)$, and Galilean boost $\left(v^{i}\right)$ defined as

$$
t \rightarrow t^{\prime}=t+a^{t}, \quad x^{i} \rightarrow x^{i}=\Lambda_{j}^{i}\left(x^{j}+a^{j}+v^{j} t\right) .
$$

The Noether equations associated with spacetime translations imply the well-known conservation of energy and momentum

$$
\begin{aligned}
\text { Energy conservation: } & \partial_{t} \epsilon^{t}+\partial_{i} \epsilon^{i}=0, \\
\text { Momentum conservation: } & \partial_{t} \pi^{i}+\partial_{j} \tau^{i j}=0 .
\end{aligned}
$$

Here $\epsilon^{t}$ is the energy density, $\epsilon^{i}$ energy flux, $\pi^{i}$ momentum density, and $\tau^{i j}$ is the stress tensor. Typically, a Galilean theory also features a conserved mass density $\rho^{t}$ and the associated flux $\rho^{i}$, leading to the continuity equation

$$
\text { Continuity equation: } \quad \partial_{t} \rho^{t}+\partial_{i} \rho^{i}=0 .
$$


The Noether equations associated with rotations and boost lead to angular momentum and centre-of-mass conservation respectively, which further enforce momentum density to equal the mass flux, $\pi^{i}=\rho^{i}$, and stress tensor to be symmetric, $\tau^{i j}=\tau^{j i} \cdot{ }^{4}$ Eq. $(2.2)$ are the generic conservation equations in a Galilean-invariant field theory. They are left invariant under the Galilean transformations (2.1) provided that various quantities transform according to

$$
\begin{array}{llrl}
\rho^{t} \rightarrow \rho^{t}, & \rho^{i} \rightarrow \Lambda_{j}^{i}\left(\rho^{j}+\rho^{t} v^{j}\right), & \tau^{i j} \rightarrow \Lambda_{k}^{i} \Lambda^{j}{ }_{l}\left(\tau^{k l}+2 v^{(k} \rho^{l)}+\rho^{t} v^{k} v^{l}\right), \\
\epsilon^{t} \rightarrow \epsilon^{t}+\frac{1}{2} \rho^{t} \vec{v}^{2}+v_{i} \rho^{i}, & \epsilon^{i} \rightarrow \Lambda_{j}^{i}\left(\epsilon^{j}+\epsilon^{t} v^{j}+\frac{1}{2} \vec{v}^{2}\left(\rho^{j}+\rho^{t} v^{j}\right)+\left(\tau^{j k}+v^{j} \rho^{k}\right) v_{k}\right) .
\end{array}
$$

To recast above equations in a more familiar form, we can define a velocity associated with the flow of mass according to $u^{i}=\rho^{i} / \rho^{t}$. It transforms as $u^{i} \rightarrow \Lambda^{i}{ }_{j}\left(u^{j}+v^{j}\right)$. We also define thermodynamic mass density $\rho$, internal/comoving thermodynamic energy density $\varepsilon$, comoving heat flux $q^{i}$, and comoving stress tensor $t^{i j}$ in the local rest frame according to ${ }^{5}$

$$
\begin{aligned}
\rho^{t} & =\rho, & \epsilon^{t} & =\varepsilon+\frac{1}{2} \rho u^{i} u_{i}, \\
\epsilon^{i} & =\left(\varepsilon+\frac{1}{2} \rho u^{i} u_{i}\right) u^{i}+t^{i j} u_{j}+q^{i}, & \tau^{i j} & =\rho u^{i} u^{j}+t^{i j} .
\end{aligned}
$$

The benefit of working with these quantities is that they are invariant under boosts. In terms of these definitions, the conservation equations turn into

$$
\begin{aligned}
\left(\partial_{t}+u^{i} \partial_{i}\right) \rho & =-\rho \partial_{i} u^{i}, \\
\left(\partial_{t}+u^{i} \partial_{i}\right) \varepsilon & =-\varepsilon \partial_{i} u^{i}-t^{i j} \partial_{i} u_{j}-\partial_{i} q^{i}, \\
\left(\partial_{t}+u^{j} \partial_{j}\right) u^{i} & =-\frac{1}{\rho} \partial_{j} t^{i j} .
\end{aligned}
$$

Eq. (2.6) are a feature of any Galilean-invariant field theory. The departure point of hydrodynamics, however, is the assumption that in a near-equilibrium state at long-enough distance and time scales and low-enough energy and momentum scales, the dynamics of the system is entirely governed by the conserved operators: mass density $\rho^{t}$, energy density $\epsilon^{t}$, and momentum density $\pi^{i}{ }^{6}$ It is convenient to reshuffle these fields and instead work

\footnotetext{
${ }^{4}$ The angular momentum and centre-of-mass conservation equations are respectively given as$$
\partial_{t}\left(\pi^{i} x^{j}-\pi^{j} x^{i}\right)+\partial_{k}\left(\tau^{k i} x^{j}-\tau^{k j} x^{i}\right)=\tau^{j i}-\tau^{i j}=0, \quad \partial_{t}\left(\rho^{t} x^{i}-\pi^{i} t\right)+\partial_{k}\left(\rho^{k} x^{i}-\tau^{k i} t\right)=\rho^{i}-\pi^{i}=0 .
$$

${ }^{5}$ The distinction between the thermodynamic mass density $\rho$ and physical mass density $\rho^{t}$ is purely notational at this point. However when coupled to background sources, this relationship gets modified with a red-shift factor. Furthermore, the definition of thermodynamic mass density is also sensitive to hydrodynamic frame redefinitions, as we will later diagnose in section 2.3. For readers familiar with relativistic hydrodynamics, this distinction is similar to the distinction between thermodynamic energy density $\epsilon$ and physical energy density $T^{t t}$ in the relativistic case.

${ }^{6}$ For this assumption to be applicable, one requires a mass-gap in the spectrum of the theory, which gaps out all the irrelevant massive degrees of freedom at low-enough energies, leaving us only with the conserved operators. In presence of massless degrees of freedom, however, one can modify the hydrodynamic equations to consistently account for the additional low energy dynamics, e.g. superfluids [55]. 
with the thermodynamic mass density $\rho$, internal energy density $\varepsilon$, and "fluid" velocity $u^{i}$. Hydrodynamics is then characterised by the most generic expressions for the comoving heat current and stress tensor in terms of the chosen variables and their spatial derivatives, i.e.

$$
q^{i}\left[\rho, \varepsilon, u^{i}, \partial_{i}\right], \quad t^{i j}\left[\rho, \varepsilon, u^{i}, \partial_{i}\right]
$$

consistent with Galilean symmetries. These are known as the hydrodynamic constitutive relations. Note that the temporal derivatives of various quantities are determined by eq. (2.6) and hence are not independent. Our assumption of near-equilibrium allows us to arrange the constitutive relations in a derivative expansion, truncated at a given order in derivatives according to the phenomenological sensitivity required. At any given order in the derivative expansion, the constitutive relations contain all the possible tensor structures made out of derivatives of $\rho, \varepsilon$, and $u^{i}$, appended with arbitrary transport coefficients as a function of $\rho$ and $\varepsilon$.

\subsubsection{Constitutive relations}

Up until this point, writing down the constitutive relations is a purely combinatorial exercise. The physical content comes in the form of certain phenomenological constraints that have to be imposed on these constitutive relations. Most important of these is the "local second law of thermodynamics" that requires that there must exist an entropy density $s^{t}$ and an associated flux $s^{i}$ such that

$$
\partial_{t} s^{t}+\partial_{i} s^{i} \geq 0
$$

At zero derivative order, entropy density is given by an arbitrary function of $\rho$ and $\varepsilon$, i.e. $s^{t}=s(\varepsilon, \rho)$. Let us define the intensive thermodynamic variables: temperature $T(\varepsilon, \rho)$, mass chemical potential $\mu(\varepsilon, \rho),{ }^{7}$ and pressure $p(\varepsilon, \rho)$ via the thermodynamic relations: local first law of thermodynamics and Euler relation respectively

$$
T \mathrm{~d} s=\mathrm{d} \varepsilon-\mu \mathrm{d} \rho, \quad p=T s+\mu \rho-\varepsilon .
$$

It is easy to check that

$$
\partial_{t} s^{t}+\partial_{i} s^{i}=-\frac{1}{T}\left(t^{i j}-p \delta^{i j}\right) \partial_{i} u_{j}-\frac{1}{T^{2}} q^{i} \partial_{i} T
$$

where we have identified the entropy flux as $s^{i}=s u^{i}+q^{i} / T$. Therefore, at leading order in derivatives, the second law constraint simply requires that

$$
t^{i j}=p \delta^{i j}+\mathcal{O}(\partial), \quad q^{i}=\mathcal{O}(\partial)
$$

We note that the comoving heat flux is zero at this order, while the comoving stress tensor is just given by the thermodynamic pressure of the fluid. Note also that entropy

\footnotetext{
${ }^{7}$ Note that $\mu$ here is the chemical potential associated with mass density, as used by Landau in [61]). For a single species fluid with mass per unit particle $m$, the particle number chemical potential is given by $m \mu$.
} 
is conserved at this order, $\partial_{t} s^{t}+\partial_{i} s^{i}=0$, hence these fluids are known as "ideal/perfect fluids". Inserting these into eq. (2.6), one recovers the well-known Euler equations of Galilean ideal hydrodynamics.

The relation $s=s(\varepsilon, \rho)$, or equivalently $\varepsilon=\varepsilon(s, \rho)$, is known as the micro-canonical equation of state of the fluid and completely characterises its constitutive relations at ideal order through the thermodynamic relations $(2.9) .{ }^{8}$ We note, however, that hydrodynamics as a physical system is better defined in the grand canonical ensemble, because a fluid element is allowed to freely exchange mass and energy with its surroundings. Keeping this in mind, we can take the fundamental dynamical fields to be $T$ and $\mu$ instead of $\varepsilon$ and $\rho$. In this case, the equation of state is given in terms of $p(T, \mu)$ instead of $\varepsilon(s, \rho)$ with the thermodynamic relations: Gibbs-Duhem relation and Euler relation respectively

$$
\mathrm{d} p=s \mathrm{~d} T+\rho \mathrm{d} \mu, \quad \varepsilon=T s+\mu \rho-p .
$$

These define $\varepsilon, \rho$, and $s$ in terms of $T$ and $\mu$.

The second law can also be used to constrain the admissible derivative corrections to the hydrodynamic equations. One finds that the entropy density remains unperturbed by one-derivative parity-preserving corrections, but is sensitive to parity-violating corrections. Focusing on $d=3$ spatial dimensions, one finds (see [31] or appendix A for more details) ${ }^{9}$

$$
\begin{aligned}
& s^{t}=s+K_{0} \epsilon^{i j k} u_{i} \partial_{j} u_{k}, \\
& s^{i}=s u^{i}+\frac{1}{T} q^{i}+K_{0} \epsilon^{i j k} u_{j}\left(\partial_{t} u_{k}+\frac{1}{2} \partial_{k} \vec{u}^{2}\right)+\left(2 K_{0} \mu+\frac{1}{2} K_{0} \vec{u}^{2}+K_{2} T\right) \epsilon^{i j k} \partial_{j} u_{k},
\end{aligned}
$$

where $K_{0}, K_{2}$ are constants. Requiring the second law to hold in generality leads to ${ }^{10}$

$$
\partial_{t} s^{t}+\partial_{i} s^{i}=-\frac{1}{T}\left(t^{i j}-p \delta^{i j}\right) \partial_{(i} u_{j)}-\frac{1}{T^{2}}\left(q^{i}-\xi_{\Omega} \epsilon^{i j k} \partial_{j} u_{k}\right) \partial_{i} T \geq 0,
$$

where $\xi_{\Omega}=T^{2} K_{2}+2 \mu T K_{0}-((\varepsilon+p) / \rho) 2 T K_{0}$ is a parity-odd transport coefficient. In obtaining this equation, we have used the ideal order Navier-Stokes equation from eq. (2.6). This results in the constitutive relations

$$
t^{i j}=p \delta^{i j}-\eta \sigma^{i j}-\zeta \delta^{i j} \partial_{k} u^{k}+\mathcal{O}\left(\partial^{2}\right), \quad q^{i}=-\kappa \partial^{i} T+\xi_{\Omega} \epsilon^{i j k} \partial_{j} u_{k}+\mathcal{O}\left(\partial^{2}\right) .
$$

where $\sigma^{i j}=\partial^{i} u^{j}+\partial^{j} u^{i}-\frac{2}{d} \delta^{i j} \partial_{k} u^{k}$ is the fluid shear tensor. The "dissipative" transport coefficients $\eta(T, \mu)$ shear viscosity, $\zeta(T, \mu)$ bulk viscosity, and $\kappa(T, \mu)$ thermal conductivity

\footnotetext{
${ }^{8}$ As a well-known example, the ideal gas "equation of state" is typically expressed as $p(\rho, T)=$ $\rho / m k_{\mathrm{B}} T$, where $m$ is the mass per particle. This, however, does not uniquely specify the thermodynamic state of the fluid; one also needs to provide $\varepsilon(\rho, T)=c_{v} \rho / m k_{\mathrm{B}} T$, where $c_{v}$ is the dimensionless specific heat capacity. Both of these can, nonetheless, be combined into a micro-canonical equation of state $\varepsilon(s, \rho)=\Lambda_{0} c_{v}(\rho / m)^{\left(c_{v}+1\right) / c_{v}} \exp \left(\frac{m s}{c_{v} \rho k_{\mathrm{B}}}-\frac{c_{v}+1}{c_{v}}\right)$ or a grand-canonical equation of state $p(T, \mu)=\Lambda_{0}^{-c_{v}}\left(k_{\mathrm{B}} T\right)^{c_{v}+1} \exp \left(\frac{m \mu}{k T}\right)$ for an arbitrary energy scale $\Lambda_{0}$.

${ }^{9}$ In fairness, implementation of the second law of thermodynamics on flat space allows for the $K_{2} T$ term eq. (2.13) to be replaced with an arbitrary function of $T$. The present form is enforced by implementing the second law in the presence of background sources, or requiring the fluid to admit an equilibrium partition function [46].

${ }^{10}$ Note the identity $\epsilon^{l j k} u^{k} \partial_{k} u_{l} \partial_{j} u_{k}=\frac{1}{2} \epsilon^{i j k} \partial_{i} \vec{u}^{2} \partial_{j} u_{k}$.
} 
contribute to entropy production and are required to be positive semi-definite on the account of the second law. On the other hand, $\xi_{\Omega}(T, \mu)$ is an "adiabatic" transport coefficient that does not lead to entropy production. Note that the parity-preserving part in eq. (2.15) is applicable for any $d$, and is standard in Galilean hydrodynamics; see e.g. [61].

We can similarly work out the constraints following from the second law of thermodynamics at arbitrarily high orders in the derivative expansion; see [57]. We can also impose additional phenomenological constraints such as the microscopic time-reversal invariance (Onsager's relations), PT invariance, or the existence of an equilibrium partition function [54].

\subsection{Coupling to sources}

We would like to introduce a set of background sources into the Galilean conservation equations (2.6) covariantly coupled to the conserved operators. To this end, we have a choice between two frameworks: Newton-Cartan (NC) backgrounds [30] or null backgrounds [46]. While the former framework is much widely used to couple to Galilean field theories [28-42], the latter has an advantage that it recasts $(d+1)$-dimensional Galilean-invariant physics in terms of $(d+2)$-dimensional relativistic physics that we understand better. Unlike the NC framework, the null background framework also explicitly manifests the Galilean (Milne) boost symmetry. In this subsection, we follow [46] to provide a brief overview of the null background framework. The relation of these results to the NC sources is reviewed later in section 5 .

\subsubsection{Null backgrounds}

It is a well-understood fact that $(d+1)$-dimensional Galilean (Bargmann) algebra can be embedded into a $(d+2)$-dimensional Poincaré algebra, seen as the set of all generators that commute with a null momenta. To be more precise, let us introduce an auxiliary direction $x^{-}$in the Galilean theory and denote the enlarged set of coordinates as $\left(x^{\mathrm{M}}\right)=\left(x^{-}, t, x^{i}\right)$. In this language, the Galilean transformations (2.1) can be understood as $x^{-}$-independent Poincare transformations written in null coordinates

$$
x^{\mathrm{M}} \rightarrow \Lambda^{\mathrm{M}}{ }_{\mathrm{N}} x^{\mathrm{N}}+a^{\mathrm{M}}, \quad \Lambda^{\mathrm{M}}{ }_{\mathrm{N}}=\left(\begin{array}{ccc}
1 & \frac{1}{2} \vec{v}^{2} & v_{j} \\
0 & 1 & 0 \\
0 & \Lambda^{i}{ }_{k} v^{k} & \Lambda^{i}{ }_{j}
\end{array}\right),
$$

with $\Lambda^{\mathrm{M}}{ }_{\mathrm{R}} \Lambda^{\mathrm{N}}{ }_{\mathrm{S}} \eta_{\mathrm{MN}}=\eta_{\mathrm{RS}}$, where $\eta_{\mathrm{MN}} \mathrm{d} x^{\mathrm{M}} \mathrm{d} x^{\mathrm{N}}=-2 \mathrm{~d} x^{-} \mathrm{d} t+\delta_{i j} \mathrm{~d} x^{i} \mathrm{~d} x^{j}$. The translations $a^{-}$ along the $x^{-}$-direction act trivially in the theory and can be understood as global U(1) "mass" transformations associated with the continuity equation. We can arrange various Galilean densities and fluxes into a $(d+2)$-dimensional energy-momentum tensor

$$
T^{\mathrm{MN}}=\left(\begin{array}{ccc}
\times & \epsilon^{t} & \epsilon^{j} \\
\epsilon^{t} & \rho^{t} & \rho^{j} \\
\epsilon^{i} & \rho^{i} & \tau^{i j}
\end{array}\right) .
$$


In terms of this, the conservation equations (2.2) are merely represented as the $(d+2)$ dimensional energy-momentum conservation

$$
\partial_{\mathrm{M}} T^{\mathrm{MN}}=0 .
$$

Note that the $\partial_{-}$derivatives in this equation are trivially zero because $T^{\mathrm{MN}}$ does not have any $x^{-}$dependence. Consequently, " $T^{--}=\times$" component in eq. (2.17) represents an irrelevant auxiliary entry that drops out of the dynamical equations. The representation (2.17) is particularly useful because the transformation properties (2.4) drastically compactify into the usual Poincaré transformation of the higher-dimensional energy-momentum tensor

$$
T^{\mathrm{MN}} \rightarrow \Lambda_{\mathrm{R}}^{\mathrm{M}} \Lambda_{\mathrm{S}}^{\mathrm{N}} T^{\mathrm{RS}} .
$$

Recast in this language, we know precisely how to couple this theory to a curved background. We promote $\eta_{\mathrm{MN}}$ to an arbitrary background metric $g_{\mathrm{MN}}$. We also lift the auxiliary coordinate direction $\partial_{-}$into a vector field $V^{\mathrm{M}} \partial_{\mathrm{M}}$. As we don't want to probe the "unphysical" component $T^{--}$of the energy-momentum tensor, we require $V^{\mathrm{M}} V^{\mathrm{N}} g_{\mathrm{MN}}=0$. We further require the metric to be independent of the auxiliary coordinate, i.e. $£_{V} g_{\mathrm{MN}}=0$. The $x^{-}$-independent Poincaré transformations get promoted to general $(d+2)$-dimensional diffeomorphisms

$$
x^{\mathrm{M}} \rightarrow x^{\mathrm{M}}(x),
$$

which act on the background fields as usual

$$
\begin{aligned}
g_{\mathrm{MN}}(x) \rightarrow g_{\mathrm{MN}}^{\prime}\left(x^{\prime}\right) & =\frac{\partial x^{\mathrm{R}}}{\partial x^{\mathrm{M}}} \frac{\partial x^{\mathrm{S}}}{\partial x^{\prime \mathrm{N}}} g_{\mathrm{RS}}(x), \\
V^{\mathrm{M}}(x) \rightarrow V^{\mathrm{M}}\left(x^{\prime}\right) & =\frac{\partial x^{\mathrm{M}}}{\partial x^{\mathrm{N}}} V^{\mathrm{N}}\left(x_{s}\right) .
\end{aligned}
$$

We can always partially fix this symmetry to set $V^{\mathrm{M}}=\delta_{-}^{\mathrm{M}}$ exactly. In this coordinate system, the residual symmetries $t \rightarrow t^{\prime}(t, \vec{x}), x^{i} \rightarrow x^{\prime i}(t, \vec{x})$ correspond to spacetime diffeomorphisms, while $x^{-} \rightarrow x^{-}-\Lambda(x)$ corresponds to local U(1) mass gauge transformations. The partial derivatives $\partial_{\mathrm{M}}$ get promoted to the covariant derivative $\nabla_{\mathrm{M}}$ associated with $g_{\mathrm{MN}}$ and the Levi-Civita symbol gets promoted to the Levi-Civita tensor $\epsilon_{-t 123}=\sqrt{-g}$. Finally, in the presence of background sources, the conservation equations (2.18) modify to

$$
\nabla_{\mathrm{M}} T^{\mathrm{MN}}=0
$$

These serve as the generalisation of eq. (2.2) to include background sources.

Given a Galilean field theory described by some action $S$, the coupling structure of $T^{\mathrm{MN}}$ to $g_{\mathrm{MN}}$ is as usual

$$
\delta S=\int \mathrm{d}^{d+2} x \sqrt{-g} \frac{1}{2} T^{\mathrm{MN}} \delta g_{\mathrm{MN}} .
$$

Eq. (2.22) follows from here provided that $S$ respects $(d+2)$-dimensional diffeomorphism invariance (that is same as $(d+1)$-dimensional Galilean invariance). Note that the integrand above is entirely independent of $x^{-}$, so the associated integral yields a trivial volume 
factor and the actual integration is only performed over the physical coordinate directions. With this in place, the retarded correlation functions of conserved currents can be computed by taking variations with respect to the sources [4]. For instance, the retarded two-point functions are given as

$$
G_{T^{\mathrm{MN}} T^{\mathrm{RS}}}^{\mathrm{R}}\left(x, x^{\prime}\right)=\left.2 \frac{\delta\left(\sqrt{-g} T^{\mathrm{MN}}(x)\right)}{\delta g_{\mathrm{RS}}\left(x^{\prime}\right)}\right|_{\text {flat }} .
$$

These formulas can be decomposed into components to arrive at the retarded two-point functions of all the Galilean observables.

\subsubsection{Hydrodynamics on curved spacetime}

We would like to couple the hydrodynamic constitutive relations to background sources. Following section 2.2.1, we first need to arrange the Galilean fluid constitutive relations into a higher dimensional "null fluid" according to eq. (2.17). Using eq. (2.5) and eq. (2.13), it can be checked that the observables of Galilean hydrodynamics and entropy current arrange themselves neatly into [46]

$$
\begin{aligned}
T^{\mathrm{MN}} & =\rho u^{\mathrm{M}} u^{\mathrm{N}}+2 \varepsilon u^{(\mathrm{M}} V^{\mathrm{N})}+2 q^{(\mathrm{M}} V^{\mathrm{N})}+t^{\mathrm{MN}}, \\
S^{\mathrm{M}} & =s u^{\mathrm{M}}+\frac{1}{T} q^{\mathrm{M}}+\Upsilon^{\mathrm{M}},
\end{aligned}
$$

with the comoving stress tensor $t^{\mathrm{MN}}$, comoving heat flux $q^{\mathrm{M}}$, and the "non-canonical" part of the entropy current $\Upsilon^{\mathrm{M}}$ are given by

$$
\begin{aligned}
t^{\mathrm{MN}} & =p \Delta^{\mathrm{MN}}-\eta \sigma^{\mathrm{MN}}-\zeta \Delta^{\mathrm{MN}} \partial_{\mathrm{R}} u^{\mathrm{R}}, \\
q^{\mathrm{M}} & =-\kappa \Delta^{\mathrm{MN}} \partial_{\mathrm{N}} T+\xi_{\Omega} \epsilon^{\mathrm{MNRST}} V_{\mathrm{N}} u_{\mathrm{R}} \partial_{\mathrm{S}} u_{\mathrm{T}}, \\
\Upsilon^{\mathrm{M}} & =\left(2 K_{0} \mu+K_{2} T\right) \epsilon^{\mathrm{MNRST}} V_{\mathrm{N}} u_{\mathrm{R}} \partial_{\mathrm{S}} u_{\mathrm{T}}-K_{0} \epsilon^{-\mathrm{MNRS}} u_{\mathrm{N}} \partial_{\mathrm{R}} u_{\mathrm{S}} .
\end{aligned}
$$

Here $V^{\mathrm{M}} \partial_{\mathrm{M}}=\partial_{-}$is the null Killing vector and $u^{\mathrm{M}} \partial_{\mathrm{M}}=\frac{1}{2} \vec{u}^{2} \partial_{-}+\partial_{t}+u^{i} \partial_{i}$ is the null fluid velocity, satisfying the normalisation conditions

$$
V^{\mathrm{M}} V_{\mathrm{M}}=u^{\mathrm{M}} u_{\mathrm{M}}=0, \quad V^{\mathrm{M}} u_{\mathrm{M}}=-1 .
$$

Also, $\Delta^{\mathrm{MN}}=\eta^{\mathrm{MN}}+2 u^{(\mathrm{M}} V^{\mathrm{N})}$ is a projector transverse to $u^{\mathrm{M}}$ and $V^{\mathrm{M}}$. We have defined the covariantised fluid shear tensor $\sigma^{\mathrm{MN}}=2 \Delta^{\mathrm{MR}} \Delta^{\mathrm{NS}}\left(\partial_{(\mathrm{R}} u_{\mathrm{S})}-1 / d P_{\mathrm{RS}} \partial_{\mathrm{T}} u^{\mathrm{T}}\right)$. Note that $t^{\mathrm{MN}}$ and $q^{\mathrm{M}}$ satisfy $t^{\mathrm{MN}} V_{\mathrm{M}}=t^{\mathrm{MN}} u_{\mathrm{N}}=q^{\mathrm{M}} V_{\mathrm{M}}=q^{\mathrm{M}} u_{\mathrm{M}}=0$ and $t^{\mathrm{MN}}=t^{\mathrm{NM}}$. The statement of the second law of thermodynamics becomes $\partial_{\mathrm{M}} S^{\mathrm{M}}=\partial_{t} s^{t}+\partial_{i} s^{i} \geq 0$. We should note that the second term in $\Upsilon^{\mathrm{M}}$ is not boost-invariant. Nonetheless, its divergence that contributes to entropy production is perfectly boost-invariant.

Coupling to curved spacetime is now straight-forward. Firstly, the normalisation conditions on the null fluid velocity (2.27) lead us to promote $u^{\mathrm{M}}$ to

$$
u^{\mathrm{M}} \partial_{\mathrm{M}}=\frac{1}{2}\left(\left(\frac{1+u^{i} g_{i-}}{g_{t-}}\right)^{2} g_{t t}-2 \frac{1+u^{i} g_{i-}}{g_{t-}} u^{i} g_{i t}+u^{i} u^{j} g_{i j}\right) \partial_{-}-\frac{1+u^{i} g_{i-}}{g_{t-}} \partial_{t}+u^{i} \partial_{i},
$$


while $\Delta^{\mathrm{MN}}=g^{\mathrm{MN}}+2 u^{(\mathrm{M}} V^{\mathrm{N})}$. As for the hydrodynamic constitutive relations, we need to promote $t^{\mathrm{MN}}, q^{\mathrm{M}}$, and $\Upsilon^{\mathrm{M}}$ into covariant expressions accordingly and obtain

$$
\begin{aligned}
t^{\mathrm{MN}} & =p \Delta^{\mathrm{MN}}-\eta \sigma^{\mathrm{MN}}-\zeta \Delta^{\mathrm{MN}} \nabla_{\mathrm{R}} u^{\mathrm{R}}, \\
q^{\mathrm{M}} & =-\kappa \Delta^{\mathrm{MN}}\left(\nabla_{\mathrm{N}} T-2 T \nabla_{[\mathrm{N}} V_{\mathrm{R}]} u^{\mathrm{R}}\right)+\xi_{\Omega} \epsilon^{\mathrm{MNRST}} V_{\mathrm{N}} u_{\mathrm{R}} \nabla_{\mathrm{S}} u_{\mathrm{T}}+\xi_{H} \epsilon^{\mathrm{MNRST}} V_{\mathrm{N}} u_{\mathrm{R}} \nabla_{\mathrm{S}} V_{\mathrm{T}}, \\
\Upsilon^{\mathrm{M}} & =\frac{a_{2}}{T} \epsilon^{\mathrm{MNRST}} V_{\mathrm{N}} u_{\mathrm{R}} \nabla_{\mathrm{S}} u_{\mathrm{T}}+\frac{a_{1}}{4 T} \epsilon^{\mathrm{MNRST}} V_{\mathrm{N}} u_{\mathrm{R}} \nabla_{\mathrm{S}} V_{\mathrm{T}}-\frac{a_{0}}{2 T} \epsilon^{-\mathrm{MNRS}} u_{\mathrm{N}} \nabla_{\mathrm{R}} u_{\mathrm{S}},
\end{aligned}
$$

where the shear tensor is now $\sigma^{\mathrm{MN}}=2 \Delta^{\mathrm{MR}} \Delta^{\mathrm{NS}}\left(\nabla_{(\mathrm{R}} u_{\mathrm{S})}-1 / d P_{\mathrm{RS}} \nabla_{\mathrm{T}} u^{\mathrm{T}}\right)$ and

$$
\begin{array}{ll}
a_{0}=2 K_{0} T, & a_{1}=2 K_{1} T^{3}+2 K_{2} T^{2} \mu+2 K_{0} T \mu^{2}, \quad a_{2}=K_{2} T^{2}+2 K_{0} T \mu, \\
\xi_{\Omega}=a_{2}-\frac{\varepsilon+p}{\rho} a_{0}, & \xi_{H}=a_{1}-\frac{\epsilon+p}{\rho} a_{2},
\end{array}
$$

with arbitrary constants $K_{0}, K_{1}, K_{2}$. In writing these, we have introduced a few new terms coupled to $2 \partial_{[\mathrm{M}} V_{\mathrm{N}]}=2 \partial_{[\mathrm{M}} g_{\mathrm{N}]}$ - that drop out on a flat background. Their coupling is fixed by the second law up to the constant $K_{1}$; see appendix A. The respective dynamical equations are, of course, given by substituting eq. (2.25) into eq. (2.22) with the constitutive relations (2.29).

\subsection{Frame transformations and thermodynamic frame}

The issue of frame transformations in hydrodynamics is a subtle one. The key idea is that while the definitions of fluid velocity, temperature, and chemical potential are well posed in equilibrium on flat space, these become hazy out-of-equilibrium or in the presence of background sources. For instance, back in section 2.1.1, we defined fluid velocity $u^{i}$ as the flow of mass $\rho^{i} / \rho^{t}$. However, one might alternatively associate $u^{i}$ with the flow of entropy/heat defined as $s^{i} / s^{t}$ instead. While in equilibrium both of these definitions agree, they are generically different as is clearly seen in eq. (2.13). Similarly, we used the thermodynamic relations (2.9) to define temperature $T$ and chemical potential $\mu$ so that the thermodynamic energy-density $\varepsilon$ and mass-density $\rho$ are identified with the physical comoving energy-density $\epsilon^{t}-\frac{1}{2} \rho \vec{u}^{2}$ and mass-density $\rho^{t}$ respectively. However, in the process, entropy-density $s^{t}$ in eq. (2.13) is no longer the same as the thermodynamic entropy density $s$. If one were to insist on identifying $s^{t}$ with $s$ by redefining $T$ and $\mu$, one of the previous identifications will need to be spoiled. Of course, any such choice is purely a matter of taste and convenience, and does not affect the physical results in any way. Nonetheless, as it turns out, choosing certain frames over others might have consequences for the well posedness of the hydrodynamic differential equations, at least in the context of relativistic hydrodynamics [63].

Let us consider a general frame transformation of the hydrodynamic variables compared to the definitions in the previous subsections

$$
T \rightarrow T+\delta T, \quad \mu \rightarrow \mu+\delta \mu, \quad u^{i} \rightarrow u^{i}+\delta u^{i},
$$

where the shifts contain at least one derivative. The last of these induces a transformation of the null fluid velocity $u^{\mathrm{M}} \rightarrow u^{\mathrm{M}}+\delta u^{\mathrm{M}}$, normalised as $V_{\mathrm{M}} \delta u^{\mathrm{M}}=u_{\mathrm{M}} \delta u^{\mathrm{M}}=0$ up to leading 
order in derivatives. The null fluid energy-momentum tensor and entropy current are expressed in terms of the new variables, up to leading order in derivatives, as

$$
\begin{aligned}
T^{\mathrm{MN}} & =(\rho+\delta \rho) u^{\mathrm{M}} u^{\mathrm{N}}+2 j^{(\mathrm{M}} u^{\mathrm{N})}+2(\varepsilon+\delta \varepsilon) u^{(\mathrm{M}} V^{\mathrm{N})}+2 q^{(\mathrm{M}} V^{\mathrm{N})}+t^{\mathrm{MN}}, \\
S^{\mathrm{M}} & =(s+\delta s) u^{\mathrm{M}}-\frac{\mu}{T} j^{\mathrm{M}}+\frac{1}{T} q^{\mathrm{M}}+\Upsilon^{\mathrm{M}},
\end{aligned}
$$

where

$$
\begin{aligned}
& \delta \rho=\frac{\partial \rho}{\partial T} \delta T+\frac{\partial \rho}{\partial \mu} \delta \mu, \quad \delta \varepsilon=\frac{\partial \varepsilon}{\partial T} \delta T+\frac{\partial \varepsilon}{\partial \mu} \delta \mu, \quad \delta s=\frac{\partial s}{\partial T} \delta T+\frac{\partial s}{\partial \mu} \delta \mu, \\
& j^{\mathrm{M}}=\rho \delta u^{\mathrm{M}}, \quad q^{\mathrm{M}}=q_{\mathrm{mass}}^{\mathrm{M}}+(\varepsilon+p) \delta u^{\mathrm{M}}, \quad t^{\mathrm{MN}}=t_{\mathrm{mass}}^{\mathrm{MN}}+(s \delta T+\rho \delta \mu) \Delta^{\mathrm{MN}} .
\end{aligned}
$$

Here $j^{\mathrm{M}}, q^{\mathrm{M}}$, and $t^{\mathrm{MN}}$ should be understood as the "comoving" mass flux, energy flux, and stress tensor with respect to the redefined fluid velocity. On the other hand, $q_{\text {mass }}^{\mathrm{M}}$ and $t_{\text {mass }}^{\mathrm{MN}}$ are the comoving energy flux and stress tensor defined with respect to the flow of mass from the previous subsections. Imposing the frame-fixing condition $T^{\mathrm{MN}} V_{\mathrm{N}}=-\rho u^{\mathrm{M}}-\varepsilon V^{\mathrm{M}}$, we recover our original results, formally referred to as the "mass frame".

Another convenient choice of hydrodynamic frame from the perspective of the EFT formalism are the so-called "thermodynamic frames". These are a class of hydrodynamic frames defined abstractly as the following: when coupled to time-independent background sources, i.e. $\partial_{t} g_{\mathrm{MN}}=0$, the trivial configuration given by $\beta^{\mathrm{M}} \equiv\left(u^{\mathrm{M}}-\mu V^{\mathrm{M}}\right) /\left(k_{\mathrm{B}} T\right)=\left(\delta_{t}^{\mathrm{M}}-\right.$ $\left.\mu_{0} V^{\mathrm{M}}\right) /\left(k_{\mathrm{B}} T_{0}\right)$ for constant $\mu_{0}$ and $T_{0}$ is a solution of the equations of motion. Functionally, the constitutive relations in a thermodynamic frame are those that satisfy the "off-shell local second law of thermodynamics":

$$
\nabla_{\mathrm{M}} S^{\mathrm{M}}+k_{\mathrm{B}} \beta_{\mathrm{N}} \nabla_{\mathrm{M}} T^{\mathrm{MN}} \geq 0
$$

without using the equations of motion. The effective action prescription naturally generates conserved currents in a thermodynamic frame. For the present case of interest, one such thermodynamic frame is given by a choice $\delta T=\delta \mu=0$ and

$$
\delta u^{\mathrm{M}}=\frac{a_{0}}{\rho} \epsilon^{\mathrm{MNRST}} V_{\mathrm{N}} u_{\mathrm{R}} \nabla_{\mathrm{S}} u_{\mathrm{T}}+\frac{a_{2}}{2 \rho} \epsilon^{\mathrm{MNRST}} V_{\mathrm{N}} u_{\mathrm{R}} H_{\mathrm{ST}} .
$$

This leads to $\delta \rho=\delta \varepsilon=\delta s=0$, along with

$$
\begin{aligned}
t^{\mathrm{MN}} & =p \Delta^{\mathrm{MN}}-\eta \sigma^{\mathrm{MN}}-\zeta \Delta^{\mathrm{MN}} \nabla_{\mathrm{R}} u^{\mathrm{R}}, \\
j^{\mathrm{M}} & =a_{0} \epsilon^{\mathrm{MNRST}} V_{\mathrm{N}} u_{\mathrm{R}} \nabla_{\mathrm{S}} u_{\mathrm{T}}+a_{2} \epsilon^{\mathrm{MNRST}} V_{\mathrm{N}} u_{\mathrm{R}} \nabla_{\mathrm{S}} V_{\mathrm{T}}, \\
q^{\mathrm{M}} & =-\kappa \Delta^{\mathrm{MN}}\left(\nabla_{\mathrm{N}} T+2 T u^{\mathrm{R}} \nabla_{[\mathrm{M}} V_{\mathrm{R}]}\right)+a_{2} \epsilon^{\mathrm{MNRST}} V_{\mathrm{N}} u_{\mathrm{R}} \nabla_{\mathrm{S}} u_{\mathrm{T}}+a_{1} \epsilon^{\mathrm{MNRST}} V_{\mathrm{N}} u_{\mathrm{R}} \nabla_{\mathrm{S}} V_{\mathrm{T}}, \\
\Upsilon^{\mathrm{M}} & =\frac{a_{2}}{T} \epsilon^{\mathrm{MNRST}} V_{\mathrm{N}} u_{\mathrm{R}} \nabla_{\mathrm{S}} u_{\mathrm{T}}+\frac{a_{1}}{4 T} \epsilon^{\mathrm{MNRST}} V_{\mathrm{N}} u_{\mathrm{R}} H_{\mathrm{ST}}-\frac{a_{0}}{2 T} \epsilon^{-\mathrm{MNRS}} u_{\mathrm{N}} \nabla_{\mathrm{R}} u_{\mathrm{S}} .
\end{aligned}
$$

We refer to this frame as the "thermodynamic mass frame", since the mass density/flux does not receive any dissipative (entropy producing) corrections. While this representation looks unarguably more involved than the mass frame expressions, the simplicity lies in the fact that all the parity-odd transport coefficients $a_{0}, a_{1}, a_{2}$ appearing here are simple 
polynomial functions of $T$ and $\mu$ given in eq. (2.30), determined up to three constants $K_{0}$, $K_{1}, K_{2}$. For reference, we note that in the absence of sources, the associated constitutive relations are given as

$$
\begin{aligned}
\rho^{t} & =\rho, \quad \epsilon^{t}=\varepsilon+\frac{1}{2} \rho \vec{u}^{2}+a_{0} \epsilon^{i j k} u_{i} \partial_{j} u_{k}, \quad \pi^{i}=\rho^{i}=\rho u^{i}+a_{0} \epsilon^{i j k} \partial_{j} u_{k}, \\
\epsilon^{i} & =\left(\varepsilon+p+\frac{1}{2} \rho \vec{u}^{2}+a_{0} \epsilon^{l j k} u_{l} \partial_{j} u_{k}-\zeta \partial_{k} u^{k}\right) u^{i}-\eta \sigma^{i j} u_{j}-\kappa \partial^{i} T+\left(a_{2}+\frac{1}{2} \vec{u}^{2} a_{0}\right) \epsilon^{i j k} \partial_{j} u_{k}, \\
\tau^{i j} & =\rho u^{i} u^{j}+p \delta^{i j}-\eta \sigma^{i j}-\zeta \delta^{i j} \partial_{k} u^{k}+2 a_{0} u^{(i} \epsilon^{j) k l} \partial_{k} u_{l} .
\end{aligned}
$$

\subsection{Generalities and adiabaticity equation}

In the preceding subsections we have reviewed a generic framework of classical Galilean hydrodynamics and discussed how to couple the hydrodynamic equations to Galileancovariant background sources. While we have focused our discussion to one-derivative hydrodynamics, the machinery follows to higher-derivative orders more or less in the same manner. Nonetheless, for our construction of effective field theories, it will be beneficial draw out some essential all-order features.

As we have seen, equations of $(d+1)$-dimensional classical Galilean hydrodynamics can be represented in terms of $(d+2)$-dimensional conservation equations (2.18). These are $d+2$ independent equations that can be solved for $d+2$ independent hydrodynamic variables (in the grand canonical ensemble): temperature $T$, chemical potential $\mu$, and null fluid velocity $u^{\mathrm{M}}$ normalised as eq. (2.27). It is convenient to package these into a single un-normalised vector field

$$
\beta^{\mathrm{M}}=\frac{1}{k_{\mathrm{B}} T}\left(u^{\mathrm{M}}-\mu V^{\mathrm{M}}\right) .
$$

The hydrodynamic constitutive relations are given as generic expressions for $T^{\mathrm{MN}}$ allowed by symmetries in terms of $\beta^{\mathrm{M}}$, the background metric $g_{\mathrm{MN}}$, and the Killing vector $V^{\mathrm{M}}$, arranged order-by-order in a derivative expansion. As such, $\beta^{\mathrm{M}}$ are some arbitrary fields chosen to describe the system and, like in any field theory, can admit arbitrary field redefinitions known as the hydrodynamic frame redefinitions; see section 2.3. A commonplace hydrodynamic frame is the so-called "mass-frame" obtained by choosing $\beta^{\mathrm{M}}$ such that $T^{\mathrm{MN}} V_{\mathrm{N}}=-\rho u^{\mathrm{M}}-\varepsilon V^{\mathrm{M}}$, where $\rho$ and $\varepsilon$ are thermodynamic mass and internal energy densities respectively defined through the thermodynamic relations eq. (2.12). The onederivative constitutive relations in this frame are given by eq. (2.25) along with eq. (2.29). However, generically, it is convenient to not restrict to this frame and leave the redefinition freedom unfixed for now.

The hydrodynamic constitutive relations are required to satisfy the second law of thermodynamics, i.e. there must exist an entropy current $S^{\mathrm{M}}$ and a quadratic form $\Delta$ satisfying

$$
\nabla_{\mathrm{M}} S^{\mathrm{M}}=k_{\mathrm{B}} \Delta \geq 0
$$

For one-derivative hydrodynamics expressed in mass frame, this is given as $S^{\mathrm{M}}=s u^{\mathrm{M}}+$ $q^{\mathrm{M}} / T+\Upsilon^{\mathrm{M}}$ with the "non-canonical" entropy current $\Upsilon^{\mathrm{M}}$ provided in eq. (2.29). This requirement is only imposed onshell, i.e. when the conservation equations are satisfied. 
Nonetheless, it can be made into an offshell statement by adding combinations of equations of motion

$$
\nabla_{\mathrm{M}} S^{\mathrm{M}}+k_{\mathrm{B}} \beta_{\mathrm{N}} \nabla_{\mathrm{M}} T^{\mathrm{MN}}=k_{\mathrm{B}} \Delta \geq 0 .
$$

In writing this equation, we have partially fixed the redefinition freedom in $\beta^{\mathrm{M}}$ by choosing it to be the multiplier for equations of motion required to make the second law an offshell statement. Such frame choices are known as the "thermodynamic frames". There is still some freedom left associated with modifying the constitutive relations with combinations of equations of motion. We know that conservation equations determine time-derivatives of various hydrodynamic fields, so we can consistently fix the residual redefinition freedom by eliminating $u^{\mathrm{M}} £_{\beta} g_{\mathrm{MN}}=u^{\mathrm{M}} \nabla_{\mathrm{M}} \beta_{\mathrm{N}}+u^{\mathrm{M}} \nabla_{\mathrm{N}} \beta_{\mathrm{M}}$ from the constitutive relations, where $£_{\beta} g_{\mathrm{MN}}=2 \nabla_{(\mathrm{M}} \beta_{\mathrm{N})}$ is the Lie derivative of $g_{\mathrm{MN}}$ along $\beta^{\mathrm{M}}$. This amounts to choosing the "thermodynamic mass frame"; the associated constitutive relations truncated to one-derivative order are given in eq. (2.32) along with eq. (2.36). The offshell second law (2.40) can be recast into a more useful form

$$
\nabla_{\mathrm{M}} N^{\mathrm{M}}=\frac{1}{2} T^{\mathrm{MN}} £_{\beta} g_{\mathrm{MN}}+\Delta, \quad \Delta \geq 0,
$$

known as the adiabaticity equation, where $N^{\mathrm{M}}=S^{\mathrm{M}} / k_{\mathrm{B}}+T^{\mathrm{MN}} \beta_{\mathrm{N}}$ is the free-energy current, ${ }^{11}$ up to terms proportional to $V^{\mathrm{M}}$ that do not enter the equation. The constitutive relations allowed by the second law of thermodynamics are the most generic solutions of eq. (2.41) for some $N^{\mathrm{M}}$ and $\Delta$. For instance, $N^{\mathrm{M}}$ for one-derivative Galilean fluid in the thermodynamic mass frame is given as $N^{\mathrm{M}}=p \beta^{\mathrm{M}}+\Upsilon^{\mathrm{M}} / k_{\mathrm{B}}$. A general classification scheme for Galilean constitutive relations based on the adiabaticity equation can be found in [57].

Based on the physical system in mind, we can also impose other phenomenological constraints on the hydrodynamic equations in addition to the second law of thermodynamics and Galilean symmetries. For instance, it is usually the case that the microscopic theory underlying the hydrodynamic system of interest has some sort of discrete time reversal invariance, denoted by $\Theta$, like T or more generally PT. ${ }^{12}$ The action of $\Theta$ on various quantities is given in table 1. Full dissipative hydrodynamics, of course, is not time reversal invariant because of entropy production, but this leads to various non-trivial constraints on the constitutive relations. For instance, constitutive relations in thermal equilibrium must be $\Theta$-invariant; this can be imposed by requiring the equilibrium partition function associated with hydrodynamics to be $\Theta$-invariant [54, 64, 65]. Choosing $\Theta=\mathrm{T}$ does not impose any constraints on the constitutive relations, while choosing $\Theta=$ T sets all the three constants $K_{0,1,2}=0$, getting rid of the parity-violating sector altogether. Another consequence of the discrete symmetry are the Onsager's reciprocity relations, which require the retarded two-point functions in eq. (2.24) to be symmetric (see [4]). This does not lead to any new constrains on one-derivative Galilean hydrodynamics.

This finishes our short course in Galilean hydrodynamics. We studied one-derivative hydrodynamics in detail and derived the associated constitutive relations using the second

\footnotetext{
${ }^{11}$ Technically, the free energy current is $-k_{\mathrm{B}} T N^{\mathrm{M}}$, but we will use this terminology for simplicity.

${ }^{12}$ Since we have only included a mass current and no charge current in our discussion, we cannot talk about CPT.
} 


\begin{tabular}{|c|c|c|c|c|}
\hline & & $\mathrm{P}$ & $\mathrm{T}$ & PT \\
\hline$X^{0}$ & & + & - & - \\
\hline$X^{i}$ & $\sigma^{i}$ & - & + & - \\
\hline$X^{-}, x^{-}, \sigma^{-}$ & $\varphi$ & + & - & - \\
\hline$u^{i}$, & & & - & + \\
\hline$T$, & & + & + & + \\
\hline$\mu, \beta^{-}, \beta^{-}$ & $\mu, \Lambda_{\beta}, \Lambda_{\beta}$ & + & + & + \\
\hline$T^{t-}, g_{t-}$ & $\epsilon^{t}, n_{t}$ & + & + & + \\
\hline$T^{i-}, g_{i-}$ & $\epsilon^{i}, n_{i}$ & - & - & + \\
\hline$T^{i j}, g_{i j}$ & $\tau^{i j}, h_{i j}$ & + & + & + \\
\hline$T^{t t}, g_{t t}$ & $\rho^{t}, b_{t}$ & + & + & + \\
\hline$T^{t i}, g_{t i}$ & $\pi^{i}=\rho^{i}, b_{i}$ & - & - & + \\
\hline
\end{tabular}

Table 1. Action of parity $(\mathrm{P})$ and time-reversal $(\mathrm{T})$ on various quantities in classical Galilean hydrodynamics and effective field theory. Schwinger-Keldysh double copies of various quantities in the effective theory, " $1 / 2$ " or " $r / a$ ", behave the same as their unlabelled versions.

law of thermodynamics. We also discussed how to couple the hydrodynamic equations to background sources, allowing us to compute retarded response functions predicted by hydrodynamics, while more generally serving as field theory sources coupled to conserved currents in the forthcoming discussion of EFT for Galilean hydrodynamics. Finally, we discussed the offshell second law of thermodynamics and the adiabaticity equation, which allows us to organise the hydrodynamic constitutive relations allowed by the second law to arbitrarily high orders in the derivative expansion. We are now ready to write down an EFT that describes stochastic fluctuations on top of classical Galilean hydrodynamics.

\section{Effective field theory for Galilean hydrodynamics}

In the previous section we reviewed a generic framework for classical Galilean hydrodynamics, expressed in terms of a one-dimensional higher relativistic "null fluid" [46]. This framework can be utilised to write down a Schwinger-Keldysh effective field theory for Galilean hydrodynamics, following its relativistic cousin developed recently [12, 13, 1517, 21] (see [21] for a review). There are two equivalent languages one can employ to describe the effective theory. One can either look at the space of individual fluid elements, each equipped with a comoving clock, and track how their spacetime position evolves as a function of the comoving time. Alternatively, one can formulate the theory on the physical spacetime and track the fluid elements occupying each spacetime point. We start with the former fluid worldvolume formulation in section 3.1, where the setup is fundamentally more natural, and subsequently move to the latter physical spacetime formulation in section 3.2, which is computationally more useful as a field theory. Irrespective of the language employed, the most interesting part of the framework are a set of SK constraints 


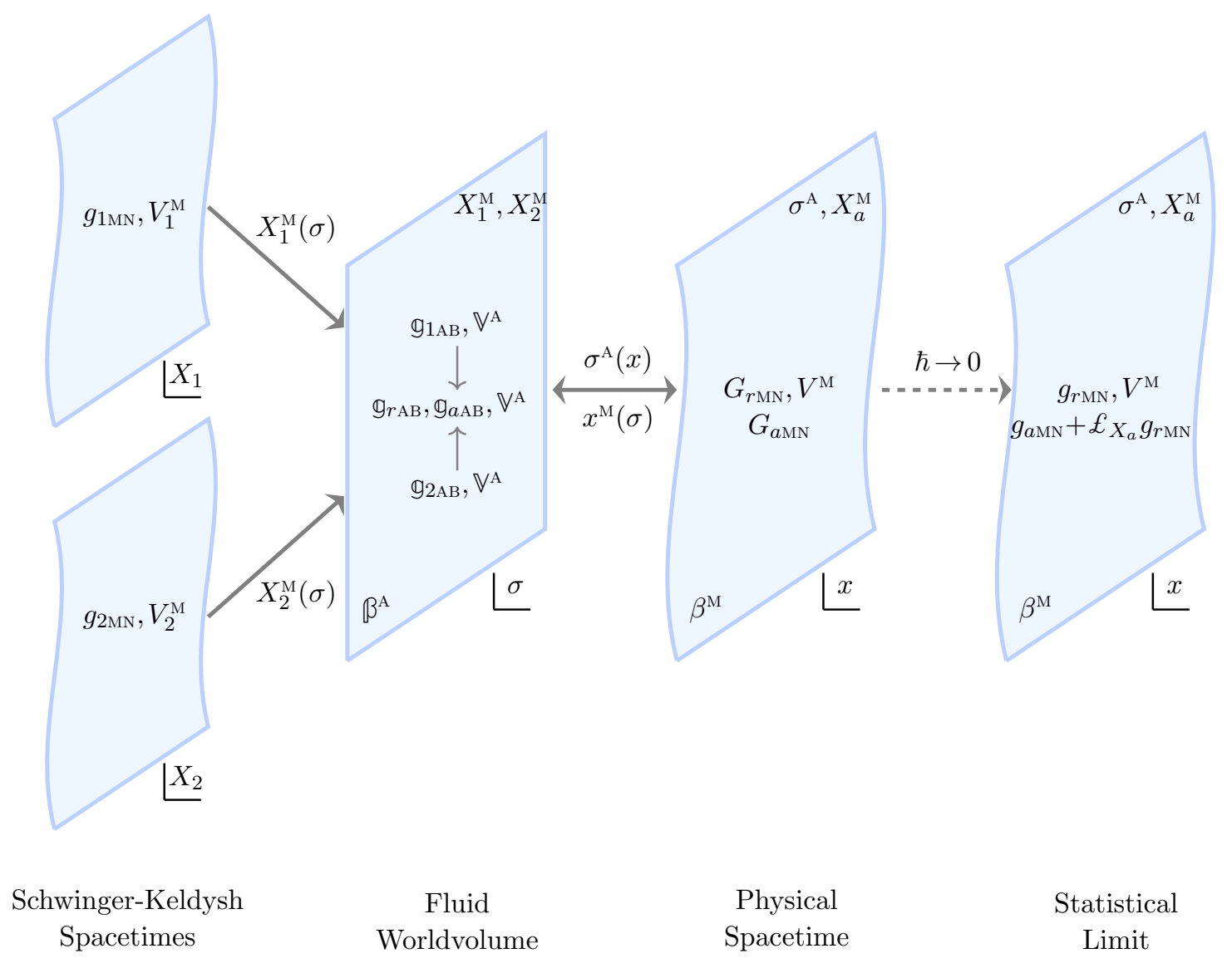

Figure 1. Schematic representation of the effective field theory framework for Galilean hydrodynamics in null background formulation.

in section 3.3, representing that the theory describes out-of-equilibrium fluctuations of a quantum system in a state of thermal equilibrium. Notably, the theory must satisfy a discrete KMS symmetry, which imposes the (non-linear) fluctuation-dissipation theorem on the hydrodynamic correlation functions and is responsible for the emergent second law of thermodynamics in the classical limit. In section 3.4, we construct the most generic effective action describing stochastic fluctuations in Galilean hydrodynamics, in a limit where all the quantum fluctuations have been suppressed, and discuss the emergence of the classical constitutive relations and second law. Later in section 4, we shall apply these ideas explicitly to one-derivative Galilean hydrodynamics.

\subsection{Fluid worldvolume formulation}

The Schwinger-Keldysh effective field theory for hydrodynamics is setup as a sigma model. We start with a $(d+2)$-dimensional "fluid worldvolume" with coordinates $\sigma^{\mathrm{A}}$. Building upon our discussion in section 2.2.1, this spacetime is required to carry a vector field $\mathbb{V}^{\mathrm{A}}(\sigma)$ characterising the auxiliary "null-direction" needed for describing a $(d+1)$-dimensional Galilean fluid. The fluid worldvolume also carries a preferred "thermal time vector" $\beta^{\mathrm{A}}(\sigma)$ that defines the reference frame associated with the global thermal state. We will require 
$\beta^{\mathrm{A}}$ to not depend on the auxiliary null direction, i.e. $£_{\mathbb{V}} \beta^{\mathrm{A}}=\mathbb{V}^{\mathrm{B}} \partial_{\mathrm{B}} \beta^{\mathrm{A}}-\beta^{\mathrm{B}} \partial_{\mathrm{B}} \mathbb{V}^{\mathrm{A}}=0$. We can always choose a basis $\left(\sigma^{\mathrm{A}}\right)=\left(\sigma^{-}, \tau, \sigma^{i}\right)$ on the fluid worldvolume such that $\mathbb{V}^{\mathrm{A}}=\delta_{-}^{\mathrm{A}}$ and $\beta^{\mathrm{A}}=\beta_{0}\left(\delta_{\tau}^{\mathrm{A}}-\mu_{0} \delta_{-}^{\mathrm{A}}\right)$, where $\beta_{0}=\left(k_{\mathrm{B}} T_{0}\right)^{-1}$ is the inverse temperature and $\mu_{0}$ the mass chemical potential of the global thermal state. In this basis, the coordinate $\tau$ can be understood as the time coordinate associated with the thermodynamic rest frame, $\sigma^{i}$ as a set of internal labels associated with each "fluid element", while the coordinate $\sigma^{-}$as the global U(1) "mass/particle number" phase of each element. However, for the majority of our discussion below, we shall leave $\mathbb{V}^{\mathrm{A}}$ and $\beta^{\mathrm{A}}$ explicit.

The fluid worldvolume carries the dynamical fields of the theory: $X_{s}^{\mathrm{M}}(\sigma)$ with $s=1,2$. These fields should be understood as the SK double copies of null spacetime coordinates of a given fluid element; see figure 1. Decomposing the coordinate fields as $X_{1,2}^{\mathrm{M}}=X_{r}^{\mathrm{M}} \pm \hbar / 2 X_{a}^{\mathrm{M}}$, the average combination $X_{r}^{\mathrm{M}}(\sigma)$ is understood as the true physical spacetime coordinates of the fluid elements, while $X_{a}^{\mathrm{M}}(\sigma)$ as the associated stochastic noise. The effective theory needs to satisfy global Galilean symmetries (2.16) independently on the two SK spacetimes. To probe the associated Noether currents, we introduce a pair of null background sources on the SK spacetimes $g_{s \mathrm{MN}}\left(X_{s}\right)$ and null vector fields $V_{s}^{\mathrm{M}}\left(X_{s}\right)$ such that $V_{s}^{\mathrm{M}} V_{s}^{\mathrm{N}} g_{s \mathrm{MN}}=0$ and $£_{V_{s}} g_{s \mathrm{MN}}=0$. The full system is now required to be invariant under $(d+2)$-dimensional diffeomorphisms

$$
X_{s}^{\mathrm{M}}(\sigma) \rightarrow X_{s}^{\mathrm{M}}\left(X_{s}(\sigma)\right),
$$

which act on the background metric and null vectors as

$$
\begin{aligned}
g_{s \mathrm{MN}}\left(X_{s}\right) \rightarrow g_{s \mathrm{MN}}^{\prime}\left(X_{s}^{\prime}\right) & =\frac{\partial X_{s}^{\mathrm{R}}}{\partial X_{s}^{\prime \mathrm{M}}} \frac{\partial X_{s}^{\mathrm{S}}}{\partial X_{s}^{\prime \mathrm{N}}} g_{s \mathrm{RS}}\left(X_{s}\right), \\
V^{\mathrm{M}}\left(X_{s}\right) \rightarrow V^{\prime \mathrm{M}}\left(X_{s}^{\prime}\right) & =\frac{\partial X_{s}^{\prime \mathrm{M}}}{\partial X_{s}^{\mathrm{N}}} V^{\mathrm{N}}\left(X_{s}\right) .
\end{aligned}
$$

We remind the reader that due to the presence of a null isometry, these are merely the $(d+2)$-dimensional representation of the $(d+1)$-dimensional local Galilean transformations. Since the choice of SK null vectors is entirely in our hands, we pick them to have the same functional form on the two SK spacetimes, i.e. $V_{1}^{\mathrm{M}}(X)=V_{2}^{\mathrm{M}}(X)$ when evaluated on the same numerical coordinates $X^{\mathrm{M}}$; this shall be useful later. To be able to describe $(d+1)$-dimensional Galilean physics, the dynamical fields $X_{s}^{\mathrm{M}}$ should only be independent functions of $\tau$ and $\sigma^{i}$, and not of the auxiliary coordinate $\sigma^{-}$. This can be fixed by requiring that the pushforward of SK null vectors $V_{s}^{\mathrm{M}}\left(X_{s}\right)$ onto the physical spacetime is the fixed worldvolume vector $\mathbb{V}^{\mathrm{M}}(\sigma)$, i.e.

$$
\mathbb{V}^{\mathrm{A}}(\sigma) \partial_{\mathrm{A}} X_{s}^{\mathrm{M}}(\sigma)=V_{s}^{\mathrm{M}}\left(X_{s}(\sigma)\right)
$$

We can also pullback the background metric over to the fluid worldvolume to define invariants under SK spacetime diffeomorphisms

$$
\mathbb{g}_{s \mathrm{AB}}(\sigma)=g_{s \mathrm{MN}}\left(X_{s}(\sigma)\right) \partial_{\mathrm{A}} X_{s}^{\mathrm{M}}(\sigma) \partial_{\mathrm{B}} X_{s}^{\mathrm{N}}(\sigma) .
$$

All the dependence on the dynamical and background fields in the effective theory enter via these invariants. Note that $\mathbb{V}^{\mathrm{A}} \mathbb{V}^{\mathrm{B}} \mathbb{G}_{s \mathrm{AB}}=0$ and $£_{\mathbb{V I}_{s \mathrm{AB}}}=0$, similar to the null background 
sources on the SK spacetimes. In fact, we can choose the average combination $\mathfrak{g}_{r_{\mathrm{AB}}}=$ $\left(g_{1 \mathrm{AB}}+g_{2 \mathrm{AB}}\right) / 2$ as a metric on the fluid worldvolume.

The choice of coordinates $\sigma^{\mathrm{A}}$ on the fluid worldvolume is entirely arbitrary and can, therefore, be arbitrarily redefined without changing any physics. This leads to the invariance of the theory under local worldvolume diffeomorphisms

$$
\sigma^{\mathrm{A}} \rightarrow \sigma^{\mathrm{A}}(\sigma)
$$

These transformations act naturally on various fluid worldvolume objects

$$
\begin{aligned}
\mathfrak{g}_{s \mathrm{AB}}(\sigma) & \rightarrow \mathbb{g}_{s \mathrm{AB}}^{\prime}\left(\sigma^{\prime}\right)=\frac{\partial \sigma^{\mathrm{C}}}{\partial \sigma^{\mathrm{A}}} \frac{\partial \sigma^{\mathrm{D}}}{\partial \sigma^{\prime \mathrm{B}}} \mathfrak{g}_{s \mathrm{CD}}(\sigma), \\
\mathbb{V}^{\mathrm{A}}(\sigma) & \rightarrow \mathbb{V}^{\prime \mathrm{A}}\left(\sigma^{\prime}\right)=\frac{\partial \sigma^{\prime \mathrm{A}}}{\partial \sigma^{\mathrm{B}}} \mathbb{V}^{\mathrm{B}}(\sigma), \quad \beta^{\mathrm{A}}(\sigma) \rightarrow \mathbb{\beta}^{\prime \mathrm{A}}\left(\sigma^{\prime}\right)=\frac{\partial \sigma^{\prime \mathrm{A}}}{\partial \sigma^{\mathrm{B}}} \mathbb{\beta}^{\mathrm{B}}(\sigma) .
\end{aligned}
$$

As mentioned previously, we can partially fix this local symmetry to explicitly set $\mathbb{V}^{\mathrm{A}}=\delta_{-}^{\mathrm{A}}$ and $\beta^{\mathrm{A}}=\beta_{0}\left(\delta_{\tau}^{\mathrm{A}}-\mu_{0} \delta_{-}^{\mathrm{A}}\right)$. The residual symmetry transformations, in this case, are the arbitrary spatial relabelling of the fluid elements $\sigma^{i} \rightarrow \sigma^{\prime i}(\vec{\sigma})$, spatial redefinitions of the local time coordinate $\tau \rightarrow \tau+f(\vec{\sigma})$, and that of the auxiliary coordinate (read U(1) mass/particle number phase) $\sigma^{-} \rightarrow \sigma^{-}+\lambda(\vec{\sigma})$. Physically, we can understand the time-independence of these transformations as the requirement that labelling scheme and phases chosen at one point in time cannot be arbitrarily changed as the fluid evolves. If we were interested in a state with spontaneously broken symmetries, like superfluids or crystals, the system could contain additional preferred coordinate directions allowing further fixing of the symmetries (3.4).

The effective action $S$ for Galilean hydrodynamics is the most generic functional made out of the constituent fields $g_{s \mathrm{MN}}, X_{s}^{\mathrm{M}}$, and their derivatives, respecting the SK spacetime symmetries (3.1) and the fluid worldvolume symmetries (3.4). Using the invariants (3.3), the effective action can be written in terms of a Lagrangian density

$$
S\left[\mathfrak{g}_{1}, \mathfrak{g}_{2} ; \mathbb{V}, \mathbb{B}\right]=\int \mathrm{d}^{d+2} \sigma \sqrt{-\mathfrak{g}_{r}} \mathcal{L}\left[\mathfrak{g}_{1}, \mathfrak{g}_{2} ; \mathbb{V}, \mathbb{\beta}\right] .
$$

The Lagrangian $\mathcal{L}$ is a scalar on the fluid worldvolume constructed by proper contraction of $\mathrm{A}, \mathrm{B}, \ldots$ indices. Notice that the integrand in eq. (3.5) is entirely independent of the fiducial $\sigma^{-}$direction, therefore the integration over this coordinate merely spits out a constant volume factor. We can define the SK double copies of the null fluid energy-momentum tensor associated with the action (3.5) by performing variations with respect to the sources

$$
T_{1}^{\mathrm{MN}}\left(X_{1}\right)=\frac{2 \hbar}{\sqrt{-g_{1}\left(X_{1}\right)}} \frac{\delta S}{\delta g_{1 \mathrm{MN}}\left(X_{1}\right)}, \quad T_{2}^{\mathrm{MN}}\left(X_{2}\right)=\frac{-2 \hbar}{\sqrt{-g_{2}\left(X_{2}\right)}} \frac{\delta S}{\delta g_{2 \mathrm{MN}}\left(X_{2}\right)} .
$$

The minus sign in the second expression stems from the second copy of the energymomentum tensor being inserted on the time-reversed part of the SK contour. We have taken $S$ to be unitless, leading to the additional factors of $\hbar$ in these formulae; this will facilitate taking a small $\hbar$ limit of the effective action later. The classical equations of motion of $X_{s}^{\mathrm{M}}$ imply the respective conservation equations for the energy-momentum tensors

$$
\nabla_{\mathrm{M}}^{s} T_{s}^{\mathrm{MN}}=0 .
$$


Here $\nabla_{\mathrm{M}}^{s}$ are covariant derivatives associated with $g_{s \mathrm{MN}}$. Hence we see that the conservation equations, used as equations of motion in classical hydrodynamics, follow from a variational principle in the effective field theory. We can use the dictionary (2.17) to read out the respective Galilean fluid observables.

\subsection{Physical spacetime formulation}

The fluid worldvolume formalism of the effective field theory discussed above is quite elegant as it makes most of the underlying structure manifest. However, for most practical purposes and to make contact with classical hydrodynamics, it is useful to translate to a physical spacetime formulation instead. To this end, let us choose the single-copy physical spacetime coordinates as the average fields $x^{\mathrm{M}} \equiv X_{r}^{\mathrm{M}}(\sigma)$. We can define the inverse maps $\sigma^{\mathrm{A}}=\sigma^{\mathrm{A}}(x)$ via $X_{r}^{\mathrm{M}}(\sigma(x))=x^{\mathrm{M}}$ and accordingly $X_{a}^{\mathrm{M}}(x)=X_{a}^{\mathrm{M}}(\sigma(x))$. With this rewriting, the set of dynamical fields in the theory are the fluid element labels $\sigma^{\mathrm{A}}(x)$ and the stochastic noise fields $X_{a}^{\mathrm{M}}(x)$ written as a function of physical spacetime coordinates.

Rewriting the invariants in eq. (3.3) in the " $r / a$ " basis as $\mathfrak{g}_{1,2 \mathrm{AB}}=\mathfrak{g}_{r_{\mathrm{AB}}} \pm \hbar / 2 \mathfrak{g}_{a \mathrm{AB}}$, we can define their pullback onto the physical spacetime as

$$
\begin{aligned}
& G_{r \mathrm{MN}}(x)=g_{r \mathrm{AB}}(\sigma(x)) \partial_{\mathrm{M}} \sigma^{\mathrm{A}}(x) \partial_{\mathrm{N}} \sigma^{\mathrm{B}}(x)=g_{r \mathrm{MN}}(x)+\mathcal{O}(\hbar), \\
& G_{a \mathrm{MN}}(x)=\mathfrak{g}_{a \mathrm{AB}}(\sigma(x)) \partial_{\mathrm{M}} \sigma^{\mathrm{A}}(x) \partial_{\mathrm{N}} \sigma^{\mathrm{B}}(x)=g_{a \mathrm{MN}}(x)+£_{X_{a}} g_{r \mathrm{MN}}(x)+\mathcal{O}(\hbar) .
\end{aligned}
$$

Here $£_{X_{a}}$ denotes a Lie derivative along $X_{a}^{\mathrm{M}}$. In the second equalities above, we have expanded the respective quantities up to leading order in $\hbar$, referred to as the "statistical limit", with $g_{1,2 \mathrm{MN}}(x)=g_{r \mathrm{MN}}(x) \pm \hbar / 2 g_{a \mathrm{MN}}(x)$ evaluated on the physical spacetime. ${ }^{13} \mathrm{We}$ can identify the average combination $g_{r \mathrm{MN}}$ with the single-copy background metric $g_{\mathrm{MN}}$ introduced in section 2.2. We can choose $G_{r \mathrm{MN}}$ as the metric on the physical spacetime. Note that $G_{r \mathrm{MN}}$ and $G_{a \mathrm{MN}}$ are not purely background fields; they are generically given by a complicated combination of background and dynamical fields. Nonetheless, in the statistical limit, the average combination $G_{r \mathrm{MN}}$ reduces to the average background metric $g_{r \mathrm{MN}}$. Similarly, using eq. (3.2), we can deduce the pushforward of the null isometry $\mathbb{V}^{\mathrm{A}}$ as

$$
V^{\mathrm{M}}(x)=\mathbb{V}^{\mathrm{A}}(\sigma(x)) \frac{\partial X_{r}^{\mathrm{M}}(\sigma(x))}{\partial \sigma^{\mathrm{A}}(x)}=\frac{1}{2}\left(V_{1}^{\mathrm{M}}(x)+V_{2}^{\mathrm{M}}(x)\right)+\mathcal{O}(\hbar) .
$$

The choice $V_{1}^{\mathrm{M}}(X)=V_{2}^{\mathrm{M}}(X)$ results in the condition $£_{V} X_{a}^{\mathrm{M}}=0$. It is easy to see that $V^{\mathrm{M}} V^{\mathrm{N}} G_{r, a \mathrm{MN}}=£_{V} G_{r, a \mathrm{MN}}=0$, providing the physical spacetime with the null background structure.

On the other hand, pushforward of the thermal time vector $\beta^{\mathrm{A}}$ onto the physical spacetime results in the "hydrodynamic fields"

$$
\beta^{\mathrm{M}}(x)=\beta^{\mathrm{A}}(\sigma(x)) \partial_{\mathrm{A}} X_{r}^{\mathrm{M}}(\sigma(x)) .
$$

\footnotetext{
${ }^{13}$ Generally, the procedure of adding/subtracting the two sets of background fields is not useful as they transform under independent symmetries. However, as we see explicitly, in the statistical $(\hbar \rightarrow 0)$ limit such an operation is well defined with the average and difference combinations being related to the pullback of the worldvolume invariants.
} 
The condition $£_{\mathbb{V}} \beta^{\mathrm{A}}=0$ leads to $£_{V} \beta^{\mathrm{M}}=0$. The conventional hydrodynamic fields: null fluid velocity $u^{\mathrm{M}}(x)$ (normalised as $u^{\mathrm{M}} u^{\mathrm{N}} G_{r \mathrm{MN}}=0, V^{\mathrm{M}} u^{\mathrm{N}} G_{r \mathrm{MN}}=-1$ ), temperature $T(x)$, and mass chemical potential $\mu(x)$ can be defined in terms of $\beta^{\mathrm{M}}$ as

$$
k_{\mathrm{B}} T(x)=\frac{1}{\beta(x)}, \quad u^{\mathrm{M}}(x)=\frac{\beta^{\mathrm{M}}(x)}{\beta(x)}+\mu(x) V^{\mathrm{M}}, \quad \mu(x)=\frac{1}{2} \frac{\beta^{\mathrm{R}}(x) \beta^{\mathrm{S}}(x) G_{r \mathrm{RS}}(x)}{\beta(x)^{2}},
$$

where $\beta(x)=-\beta^{\mathrm{M}}(x) V^{\mathrm{N}}(x) G_{r \mathrm{MN}}(x)$. Note that in the statistical limit, $G_{r \mathrm{MN}}, G_{a \mathrm{MN}}$, and $V^{\mathrm{M}}$ are entirely independent of the non-stochastic dynamical fields $\sigma^{\mathrm{A}}(x)$ and all dependence thereof comes only via the hydrodynamic fields. This justifies the validity of the choice of degrees of freedom in classical hydrodynamics.

Working in the physical spacetime formulation, the fluid worldvolume symmetries (3.4) are explicitly realised. As a payoff, however, we need to implement the "average part" 14 of the Schwinger-Keldysh spacetime symmetries (3.1), pulled down to the physical spacetime through the coordinate maps $x^{\mathrm{M}}=X_{r}^{\mathrm{M}}(\sigma)$, leading to physical spacetime diffeomorphisms

$$
x^{\mathrm{M}} \rightarrow x^{\mathrm{M}}(x) .
$$

This acts on various physical spacetime fields in the theory as expected

$$
\begin{aligned}
G_{r, a \mathrm{MN}}(x) & \rightarrow G_{r, a \mathrm{MN}}^{\prime}\left(x^{\prime}\right)=\frac{\partial x^{\mathrm{R}}}{\partial x^{\prime \mathrm{M}}} \frac{\partial x^{\mathrm{S}}}{\partial x^{\mathrm{N}}} G_{r, a \mathrm{RS}}(x), \\
V^{\mathrm{M}}(x) & \rightarrow V^{\prime \mathrm{M}}\left(x^{\prime}\right)=\frac{\partial x^{\prime \mathrm{M}}}{\partial x^{\mathrm{N}}} V^{\mathrm{N}}(x), \quad \beta^{\mathrm{M}}(x) \rightarrow \beta^{\prime \mathrm{M}}\left(x^{\prime}\right)=\frac{\partial x^{\prime \mathrm{M}}}{\partial x^{\mathrm{N}}} \beta^{\mathrm{N}}(x) .
\end{aligned}
$$

Note that, unlike eq. (3.1), we only have a single copy of physical spacetime diffeomorphisms here.

The effective action (3.5) can also be translated into a physical spacetime representation. One can treat $\sigma^{\mathrm{A}} \rightarrow x^{\mathrm{M}}=X_{r}^{\mathrm{M}}(\sigma)$ as a diffeomorphism and represent the coordinate transformed effective action (3.5) as

$$
S\left[G_{r}, G_{a} ; \beta, V\right]=\int \mathrm{d}^{d+2} x \sqrt{-G_{r}} \mathcal{L}\left[G_{r}, G_{a} ; \beta, V\right] .
$$

The Lagrangian $\mathcal{L}$ is a scalar with appropriate contraction of $\mathrm{M}, \mathrm{N}, \ldots$ indices among the constituent fields. Given the effective action, we can define the " $r / a$ " basis of null background conserved energy-momentum tensors according to

$$
\begin{aligned}
& T_{r}^{\mathrm{MN}}(x)=\frac{2}{\sqrt{-G_{r}(x)}} \frac{\delta S}{\delta g_{a \mathrm{MN}}(x)}=\frac{1 / 2}{\sqrt{-G_{r}(x)}}\left(\sqrt{-g_{1}(x)} T_{1}^{\mathrm{MN}}(x)+\sqrt{-g_{2}(x)} T_{2}^{\mathrm{MN}}(x)\right), \\
& T_{a}^{\mathrm{MN}}(x)=\frac{2}{\sqrt{-G_{r}(x)}} \frac{\delta S}{\delta g_{r \mathrm{MN}}(x)}=\frac{1 / \hbar}{\sqrt{-G_{r}(x)}}\left(\sqrt{-g_{1}(x)} T_{1}^{\mathrm{MN}}(x)-\sqrt{-g_{2}(x)} T_{2}^{\mathrm{MN}}(x)\right) .
\end{aligned}
$$

\footnotetext{
${ }^{14}$ Explicitly in terms of eq. (3.1), one finds$$
x^{\prime \mathrm{M}}(x)=\frac{1}{2}\left(X_{1}^{\prime \mathrm{M}}\left(x+\hbar / 2 X_{a}(x)\right)+X_{2}^{\prime \mathrm{M}}\left(x-\hbar / 2 X_{a}(x)\right)\right)=\frac{1}{2}\left(X_{1}^{\prime \mathrm{M}}(x)+X_{2}^{\prime \mathrm{M}}(x)\right)+\mathcal{O}(\hbar) .
$$

In the statistical limit, these turn into the average combinations of the Schwinger-Keldysh spacetime transformations.
} 
The second equalities here follow from the expressions in eq. (3.6). The average combination $T_{r}^{\mathrm{MN}}$ is understood as the physical null background energy-momentum tensor, while the difference combination $T_{a}^{\mathrm{MN}}$ is understood as the associated stochastic noise. While both of these operators are conserved on flat spacetime, they are not individually conserved in the presence of background sources. Nonetheless, in the statistical limit, the physical average combination satisfies the classical conservation equation (2.22). The classical energy-momentum tensor $T^{\mathrm{MN}}$ from section 2 is obtained from $T_{r}^{\mathrm{MN}}$ by going to the statistical $(\hbar \rightarrow 0)$ limit and switching off all the stochastic " $a$ " type fields $g_{a \mathrm{MN}}$ and $X_{a}^{\mathrm{M}}$.

\subsection{Schwinger-Keldysh generating functional}

Let us momentarily return to our original starting point of the EFT on the fluid worldvolume. We argued that Galilean hydrodynamics can be described by an effective action (3.5), written as a functional of two copies of background fields $g_{s \mathrm{sN}}\left(X_{s}\right)$ and two copies of dynamical fields $X_{s}^{\mathrm{M}}(\sigma)$, consistent with certain spacetime symmetries. The generating functional for the theory, which allows us to compute out-of-equilibrium thermal correlators of various hydrodynamic operators, is obtained by the path integral

$$
\exp W\left[g_{1}, g_{2} ; V_{1}, V_{2}\right]=\int \mathcal{D} X_{1} \mathcal{D} X_{2} \exp \left(i S\left[g_{1}, g_{2} ; \mathbb{B}, \mathbb{V}\right]\right)
$$

We have absorbed the conventional weight factor of $1 / \hbar$ in the exponential within the definition of $S$ making it unitless. This will allow the path integral to have a well-defined statistical $(\hbar \rightarrow 0)$ limit. In the physical spacetime representation, we can equivalently write $^{15}$

$$
\exp W\left[g_{r}, g_{a} ; V\right]=\int \mathcal{D} \sigma \mathcal{D} X_{a} \exp \left(i S\left[G_{r}, G_{a} ; \beta, V\right]\right)
$$

This generating functional can be used to compute out-of-equilibrium thermal correlators of conserved Galilean densities and fluxes in the " $r / a$ " basis through

$$
G_{T_{\alpha}^{\mathrm{MN}} \ldots}(x, \ldots)=i^{n_{a}}\left(\frac{-i \delta}{\delta g_{\bar{\alpha} \mathrm{MN}}(x)} \ldots\right) W\left[g_{r}, g_{a}\right]
$$

where $\alpha=r, a$ and $\bar{\alpha}=a, r$ is its conjugate, while $n_{a}$ is the number of $a$ type fields in the correlator on the left. Following general SK machinery (see e.g. [22]), the correlators of the type " $r a \ldots a$ " are interpreted as the fully retarded correlation functions, " $a$...ar" as the fully advanced, "rr...r" as the symmetric correlation functions, and various other timeordering schemes in between. For instance, the retarded, advanced, and symmetric two

\footnotetext{
${ }^{15}$ In principle, one needs to be careful about the Jacobian of the field redefinition. However, as the transformation from $X_{1,2}^{\mu}$ to $X_{a}^{\mu}, \sigma^{\mathrm{A}}$ is purely algebraic, i.e. does not include any derivatives, the ghosts obtained by exponentiating the Jacobian do not propagate and can be ignored [66].
} 
point functions can be computed via

$$
\begin{aligned}
& G_{T^{\mathrm{MN}} T^{\mathrm{RS}}}^{\mathrm{R}}\left(x, x^{\prime}\right)=G_{T_{r}^{\mathrm{MN}} T_{a}^{\mathrm{RS}}}\left(x, x^{\prime}\right)=\frac{-i \delta^{2} W}{\delta g_{a \mathrm{MN}}(x) \delta g_{r \mathrm{RS}}\left(x^{\prime}\right)}, \\
& G_{T^{\mathrm{MN}} T^{\mathrm{RS}}}^{\mathrm{A}}\left(x, x^{\prime}\right)=G_{T_{a}^{\mathrm{MN}} T_{r}^{\mathrm{RS}}}\left(x, x^{\prime}\right)=\frac{-i \delta^{2} W}{\delta g_{r \mathrm{MN}}(x) \delta g_{a \mathrm{RS}}\left(x^{\prime}\right)}, \\
& G_{T^{\mathrm{MN}} T^{\mathrm{RS}}}^{\mathrm{S}}\left(x, x^{\prime}\right)=G_{T_{r}^{\mathrm{MN}} T_{r}^{\mathrm{RS}}}\left(x, x^{\prime}\right)=\frac{-i \delta^{2} W}{\delta g_{a \mathrm{MN}}(x) \delta g_{a \mathrm{RS}}\left(x^{\prime}\right)} .
\end{aligned}
$$

The retarded correlator can be compared to the expression (2.24) from classical hydrodynamics.

We have strategically avoided mentioning the "aa" correlator in eq. (3.18); in general, all the correlators of the type " $a a \ldots a$ " must be zero for $W$ describing a bona fide out-of-equilibrium thermal field theory. Similarly, the retarded and advanced correlators, in momentum space, must be complex conjugates of each other (in momentum space). On the other hand, the retarded and symmetric correlators must be related by the fluctuation-dissipation theorem. There are similar constraints for higher-point functions as well; see [22]. These are compactly represented in terms of the generating functional as

$$
\begin{aligned}
W^{*}\left[g_{1}, g_{2} ; V_{1}, V_{2}\right] & =W\left[g_{2}, g_{1} ; V_{2}, V_{1}\right], \quad W[g, g ; V, V]=0, \quad \operatorname{Re} W\left[g_{1}, g_{2} ; V_{1}, V_{2}\right] \leq 0 \\
W\left[g_{1}, g_{2} ; V_{1}, V_{2}\right] & =W\left[\tilde{g}_{1}, \tilde{g}_{2} ; \tilde{V}_{1}, \tilde{V}_{2}\right] .
\end{aligned}
$$

The last of these is known as the KMS condition, with KMS conjugation of the background fields defined as

$$
\begin{aligned}
\tilde{g}_{1 \mathrm{MN}}(x) & =\Theta g_{1 \mathrm{MN}}(x), & \tilde{g}_{2 \mathrm{MN}}(x) & =\Theta g_{2 \mathrm{MN}}\left(t+i \hbar \beta_{0}, \vec{x}\right), \\
\tilde{V}_{1}^{\mathrm{M}}(x) & =\Theta V_{1}^{\mathrm{M}}(x), & \tilde{V}_{2}^{\mathrm{M}}(x) & =\Theta V_{2}^{\mathrm{M}}\left(t+i \hbar \beta_{0}, \vec{x}\right) .
\end{aligned}
$$

Here $\beta_{0}=\left(k_{\mathrm{B}} T_{0}\right)^{-1}$ is the constant inverse temperature of the global thermal state and $\Theta$ represents a discrete symmetry involving a time-flip that the system might enjoy, for example time-reversal $\mathrm{T}$ or spacetime parity PT; see table 1.

The conditions (3.19) can be implemented in the field theory by requiring the effective action to obey the constraints $[12]^{16}$

$$
\begin{aligned}
S^{*}\left[\mathfrak{g}_{1}, \mathfrak{g}_{2} ; \mathbb{B}, \mathbb{V}\right] & =-S\left[\mathfrak{g}_{2}, \mathfrak{g}_{1} ; \mathbb{B}, \mathbb{V}\right], \\
S[\mathfrak{g}, \mathbb{g} ; \mathbb{B}, \mathbb{V}] & =0, \\
\operatorname{Im} S\left[\mathfrak{g}_{1}, \mathfrak{g}_{2} ; \mathbb{B}, \mathbb{V}\right] & \geq 0, \\
S\left[\mathfrak{g}_{1}, \mathfrak{g}_{2} ; \mathbb{B}, \mathbb{V}\right] & =S\left[\tilde{\mathfrak{g}}_{1}, \tilde{\mathfrak{g}}_{2} ; \tilde{\mathbb{B}}, \tilde{\mathbb{V}}\right],
\end{aligned}
$$

where the KMS conjugation of the respective fields is defined according to

$$
\begin{aligned}
\tilde{X}_{1}^{\mathrm{M}}(\sigma) & =\Theta X_{1}^{\mathrm{M}}(\sigma), & \tilde{X}_{2}^{\mathrm{M}}(\sigma) & =\Theta X_{2}^{\mathrm{M}}(\sigma+i \hbar \Theta \beta(\sigma))-i \hbar \beta_{0} \delta_{t}^{\mathrm{M}}, \\
\tilde{\mathbb{V}}^{\mathrm{A}}(\sigma) & =\Theta \mathbb{V}^{\mathrm{A}}(\sigma), & \tilde{\mathbb{\beta}}^{\mathrm{A}}(\sigma) & =\Theta \beta^{\mathrm{A}}(\sigma),
\end{aligned}
$$

\footnotetext{
${ }^{16}$ See footnote 2.
} 
leading to

$$
\begin{aligned}
& \tilde{\mathfrak{g}}_{1 \mathrm{AB}}(\sigma)=\Theta \mathfrak{g}_{1 \mathrm{AB}}(\sigma), \\
& \tilde{\mathfrak{g}}_{2 \mathrm{AB}}(\sigma)=\partial_{\mathrm{A}}\left(\sigma^{\mathrm{C}}+i \hbar \Theta \beta^{\mathrm{C}}(\sigma)\right) \partial_{\mathrm{B}}\left(\sigma^{\mathrm{D}}+i \hbar \Theta \beta^{\mathrm{D}}(\sigma)\right) \Theta \mathrm{g}_{2 \mathrm{CD}}(\sigma+i \hbar \Theta \beta(\sigma)) .
\end{aligned}
$$

The argument $(\sigma+i \hbar \Theta \beta(\sigma))$ should be understood as a vector, i.e. $\sigma^{\mathrm{A}}+i \hbar \Theta \beta^{\mathrm{A}}(\sigma)$. The condition $£_{\mathbb{V}} \beta^{\mathrm{A}}=0$ ensures that eq. (3.2) remains satisfied by the conjugated quantities. The KMS conjugation in the " 1 " sector is merely a $\Theta$-conjugation, while in the " 2 " sector it is given by a $\Theta$-conjugation followed by a diffeomorphism along $i \hbar \Theta \beta^{\mathrm{M}}(\sigma)$. The KMS transformation in the "2" sector is highly non-trivial and is quite hard to implement in its full generality. Fortunately, the transformation becomes better-behaved in the small $\hbar$ limit

$$
\tilde{\mathfrak{g}}_{1 \mathrm{AB}}(\sigma)=\Theta \mathfrak{g}_{1 \mathrm{AB}}(\sigma), \quad \tilde{\mathfrak{g}}_{2 \mathrm{AB}}(\sigma)=\Theta_{\mathfrak{g}_{2 \mathrm{AB}}}(\sigma)-i \hbar \Theta £_{\beta} \mathbb{g}_{2 \mathrm{AB}}(\sigma),
$$

which is much easier to implement in the effective theory than the full quantum version. See appendix A of [14] for more discussion.

The constraints (3.21) can also be stated in the physical spacetime language, i.e.

$$
\begin{aligned}
S^{*}\left[G_{r}, G_{a} ; \beta, V\right] & =-S\left[G_{r},-G_{a} ; \beta, V\right], \\
S\left[G_{r}, G_{a}\right. & =0 ; \beta, V]=0, \\
\operatorname{Im} S\left[G_{r}, G_{a} ; \beta, V\right] & \geq 0, \\
S\left[G_{r}, G_{a} ; \beta, V\right] & =S\left[\tilde{G}_{r}, \tilde{G}_{a} ; \tilde{\beta}, \tilde{V}\right],
\end{aligned}
$$

where the KMS conjugation of various fields can be derived using eq. (3.22). However, the full quantum expressions are quite complicated as the physical coordinates $x^{\mathrm{M}}=X_{r}^{\mathrm{M}}(\sigma)$ non-trivially mix the "1" and "2" type spacetime fields. Nonetheless, in the statistical limit, we can derive

$$
\begin{aligned}
\tilde{\sigma}^{\mathrm{A}}(x) & =\Theta \sigma^{\mathrm{A}}(x)+\mathcal{O}(\hbar), & \tilde{X}_{a}^{\mathrm{M}}(x) & =\Theta X_{a}^{\mathrm{M}}(x)-i \Theta \beta^{\mathrm{M}}(x)+i \beta_{0} \delta_{t}^{\mathrm{M}}+\mathcal{O}(\hbar), \\
\tilde{g}_{r \mathrm{MN}}(x) & =\Theta g_{r \mathrm{MN}}(x)+\mathcal{O}(\hbar), & \tilde{g}_{a \mathrm{MN}}(x) & =\Theta g_{a \mathrm{MN}}(x)+i \beta_{0} \Theta \partial_{t} g_{r \mathrm{MN}}(x)+\mathcal{O}(\hbar), \\
\tilde{G}_{r \mathrm{MN}}(x) & =\Theta G_{r \mathrm{MN}}(x)+\mathcal{O}(\hbar), & \tilde{G}_{a \mathrm{MN}}(x) & =\Theta G_{a \mathrm{MN}}(x)+i \Theta £_{\beta} G_{r \mathrm{MN}}(x)+\mathcal{O}(\hbar), \\
\tilde{V}^{\mathrm{M}}(x) & \rightarrow \Theta V^{\mathrm{M}}(x)+\mathcal{O}(\hbar), & \tilde{\beta}^{\mathrm{M}}(x) & \rightarrow \Theta \beta^{\mathrm{M}}(x)+\mathcal{O}(\hbar) .
\end{aligned}
$$

Recall that in small $\hbar$ limit, $G_{r \mathrm{MN}}(x)=g_{r \mathrm{MN}}(x)+\mathcal{O}(\hbar)$. Note that the Lie derivative operator $£_{\beta}$ is odd under $\Theta$. Together, these requirements constrain the most generic form of an EFT for Galilean hydrodynamics. At the classical level, these constraints conspire to ensure that the Onsager's relations and local second law of thermodynamics are satisfied order-by-order in the derivative expansion [14]; see the next subsection.

\subsection{Explicit effective action and emergent second law}

We would like to write down the most generic effective action describing Galilean hydrodynamics guided by the structure outlined above. Let us work in the physical spacetime formulation, wherein the effective action must respect the physical spacetime symmetries (3.12) and SK constraints (3.25); note that the fluid worldvolume symmetries are 
manifestly preserved in the physical spacetime representation. The discussion in the fluid worldvolume language is analogous. As mentioned in section 3.3, we do not have the tools to implement the full non-local KMS symmetry in the quantum regime, so we are forced to work in the statistical $(\hbar \rightarrow 0)$ limit. In this limit, all the quantum fluctuations freeze out and we are only left with statistical/stochastic fluctuations. Even so, the structure of the simplified effective theory is extremely rich and is suitable to describe the effect of stochastic interactions in hydrodynamic correlation functions. One could, in principle, reintroduce quantum effects perturbatively in $\hbar$, which we shall not explore in the present work.

The most generic Lagrangian consistent with eq. (3.25b) can be arranged in a power series in $G_{a}$ starting from the linear term, with factors of $i$ chosen for consistency with eq. (3.25a). We have

$$
\mathcal{L}=\sum_{m=1}^{\infty} \frac{(-i)^{m+1}}{2^{m}} \mathcal{F}_{m}(\underbrace{G_{a}, G_{a}, \ldots}_{\times m})+\mathcal{O}(\hbar) .
$$

Here $\mathcal{F}_{m}(\circ, \cdots)$ are a set of totally symmetric real multi-linear operators made out of $G_{r \mathrm{MN}}$, $V^{\mathrm{M}}$ and $\beta^{\mathrm{M}}$, allowing $m$ number of arguments. The underbrace notation is meant to denote a repeated set of arguments. We can perform a change of basis on the operators given by

$$
\mathcal{F}_{m}(\underbrace{\circ, \ldots}_{\times m})=\sum_{n=m}^{2 m+1} \frac{c_{m n}}{2^{n-m}} \mathcal{D}_{n}(\underbrace{\circ, \ldots}_{\times m}, \underbrace{\sum_{\beta} G_{r}, \ldots}_{\times n-m}), \quad c_{m n}=\left\{\begin{array}{ll}
(-)^{n / 2+1}\left(\begin{array}{c}
n / 2 \\
n-m
\end{array}\right) & n \text { even } \\
(-)^{(n+1) / 2} \frac{m+1}{n+1}\left(\begin{array}{c}
(n+1) / 2 \\
n-m
\end{array}\right) & n \text { odd }
\end{array},\right.
$$

where $\mathcal{D}_{m}(\circ, \cdots)$, again, are symmetric real multi-linear maps similar to $\mathcal{F}_{m}(\circ, \cdots)$. Manipulating the double summations, one finds that this operation recasts the Lagrangian into

$$
\begin{aligned}
\mathcal{L}= & \frac{1}{2} \mathcal{D}_{1}\left(G_{a}\right)+i \sum_{n=1}^{\infty} \frac{1}{2^{2 n}} \mathcal{D}_{2 n}(\underbrace{G_{a}, \ldots}_{\times n}, \underbrace{G_{a}+i £_{\beta} G_{r}, \ldots}_{\times n}) \\
& +\sum_{n=1}^{\infty} \frac{1}{2^{2 n+1}} \mathcal{D}_{2 n+1}(G_{a}+\frac{i}{2} £_{\beta} G_{r}, \underbrace{G_{a}, \ldots}_{\times n}, \underbrace{G_{a}+i £_{\beta} G_{r}, \ldots}_{\times n})+\mathcal{O}(\hbar) .
\end{aligned}
$$

This comprises the most general effective action for Galilean hydrodynamics in the statistical limit. The utility of this form is that under the KMS conjugation (3.26), the arguments of the operators under the summation map to each other up to a $\Theta$-conjugation, i.e. the $n$ instances of $G_{a} \rightarrow \Theta G_{a}+i \Theta £_{\beta} G_{r}$ and $G_{a}+i £_{\beta} G_{r} \rightarrow \Theta G_{a}$ map to each other, while $G_{a}+\frac{i}{2} £_{\beta} G_{r} \rightarrow \Theta G_{a}+\frac{i}{2} \Theta £_{\beta} G_{r}$ maps to itself. Only the $\mathcal{D}_{1}\left(G_{a}\right)$ term outside the summation poses an exception to this general rule and leads to our first constraint

$$
\frac{1}{2} \mathcal{D}_{1}\left(£_{\beta} G_{r}\right)=\nabla_{\mathrm{M}} \mathcal{N}_{0}^{\mathrm{M}} \quad \text { for some vector } \mathcal{N}_{0}^{\mathrm{M}},
$$

This ensures that the term does not generate a $G_{a}$-independent piece under KMS transformation (up to a total derivative), in accordance with eq. (3.25b). Since the operators 
$\mathcal{D}_{m}$ are made out of only " $r$ " type fields, KMS merely acts on these as a $\Theta$-conjugation. Given that the arguments already map to each other up to a $\Theta$-conjugation under KMS, in order to respect eq. (3.25d) we just need to ensure that $\mathcal{D}_{m}$ has the right sign under $\Theta$. We can express this requirement as

$$
\mathcal{D}_{m}\left(G_{a}, G_{a}, \ldots\right) \text { is } \Theta \text {-even } \forall m \text {. }
$$

Note that $£_{\beta}$ is a $\Theta$-odd operation, therefore swapping any of the $G_{a}$ 's in the arguments above with $i £_{\beta} G_{r}$ flips the $\Theta$-parity of the resultant term. As a consequence, the Lagrangian (3.29) as a whole does not have a definite sign under $\Theta$. This should be expected due to the presence of dissipation. This only leaves us with the inequality constraint in eq. (3.25c), leading to

$$
\frac{(-1)^{m+1}}{2^{2 m}} \mathcal{F}_{2 m}(\underbrace{G_{a}, \ldots}_{\times 2 m})=(-1)^{m+1} \sum_{n=2 m}^{4 m+1} \frac{c_{2 m, n}}{2^{n}} \mathcal{D}_{n}(\underbrace{G_{a}, \ldots}_{\times 2 m}, \underbrace{\sum_{\beta} G_{r}, \ldots}_{\times n-2 m}) \geq 0,
$$

for arbitrary field configurations. In practise, this condition can be implemented order-byorder in $G_{a}$ and derivative expansion [67,68], leading to a simple condition

$$
\left.\mathcal{D}_{2}(G, G)\right|_{\text {leading order }} \geq 0 \text { for arbitrary } G_{\mathrm{MN}},
$$

If the leading derivative piece in $\mathcal{D}_{2}$ happens to be zero for a system (which amounts to zero viscosities and conductivity), the requirement can, in principle, shift to higher order in derivatives or $G_{a}$, leading to different equality/inequality constraints; see [19] for an analogue of this for relativistic fluids.

The constraints (3.30) are the field theory realisation of the second law of thermodynamics and Onsager's relations. To see this, let us perform integration by parts to define

$$
\mathcal{D}_{m}(G, \underbrace{\circ, \ldots}_{\times m-1})=G_{\mathrm{MN}} \mathcal{T}_{m}^{\mathrm{MN}}(\underbrace{\circ, \ldots}_{\times m-1})+\nabla_{\mathrm{M}} \mathcal{N}_{m}^{\mathrm{M}}(G ; \underbrace{\circ, \ldots}_{\times m-1}),
$$

for arbitrary symmetric tensor $G_{\mathrm{MN}}$. This equation essentially says that if $\mathcal{D}_{m}$ acts on its argument $G_{\mathrm{MN}}$ as a differential operator, we can remove the derivatives by adding a total derivative term. The classical constitutive relations $T^{\mathrm{MN}}$ are defined as the average quantities $T_{r}^{\mathrm{MN}}$ evaluated on a configuration with zero "a" type fields $X_{a}^{\mathrm{M}}=g_{a \mathrm{MN}}=0$. Using the variational formulae (3.14) and the definitions (3.31), we can obtain

$$
T^{\mathrm{MN}}=\mathcal{T}_{1}^{\mathrm{MN}}-\frac{1}{2} \mathcal{T}_{2}^{\mathrm{MN}}\left(£_{\beta} G_{r}\right)-\frac{1}{8} \mathcal{T}_{3}^{\mathrm{MN}}\left(£_{\beta} G_{r}, £_{\beta} G_{r}\right) .
$$

Note that the operators $\mathcal{D}_{n}$ for $n>3$ do not contribute to the classical constitutive relations. Using (3.31) again, one can derive an identity satisfied by these constitutive relations

$$
\frac{1}{2} T^{\mathrm{MN}} £_{\beta} g_{\mathrm{MN}}=\nabla_{\mathrm{M}} N^{\mathrm{M}}-\Delta, \quad \Delta \geq 0,
$$

where

$$
\begin{aligned}
N^{\mathrm{M}} & =\mathcal{N}_{0}^{\mathrm{M}}+\frac{1}{2} \mathcal{N}_{1}^{\mathrm{M}}\left(£_{\beta} G_{r}\right)-\frac{1}{4} \mathcal{N}_{2}^{\mathrm{M}}\left(£_{\beta} G_{r}, £_{\beta} G_{r}\right)-\frac{1}{16} \mathcal{N}_{3}^{\mathrm{M}}\left(£_{\beta} G_{r}, £_{\beta} G_{r}, £_{\beta} G_{r}\right), \\
\Delta & =\frac{1}{4} \mathcal{D}_{2}\left(£_{\beta} G_{r}, £_{\beta} G_{r}\right)+\frac{1}{16} \mathcal{D}_{3}\left(£_{\beta} G_{r}, £_{\beta} G_{r}, £_{\beta} G_{r}\right) .
\end{aligned}
$$


In obtaining this, we have used the condition (3.30a) and the identification of $G_{r \text { MN }}$ with the single-copy background metric $g_{\mathrm{MN}}$ in the statistical limit. The positivity of $\Delta$, within a derivative expansion, follows from the condition (3.30c). The condition (3.33) is precisely the adiabaticity condition (2.41) of Galilean hydrodynamics. Consequently, the entropy current defined as $S^{\mathrm{M}}=k_{\mathrm{B}} N^{\mathrm{M}}-T^{\mathrm{MN}} u_{\mathrm{N}} / T$ follows the second law of thermodynamics, $\nabla_{\mathrm{M}} S^{\mathrm{M}}=k_{\mathrm{B}} \Delta \geq 0$, onshell. We have, therefore, arrived at a derivation of the second law of thermodynamics within the hydrodynamic field theoretic framework, originally due to [12] for relativistic fluids.

Finally, the condition (3.30b), along with eq. (3.31) and eq. (3.32), implies that the contribution from $\mathcal{D}_{1}$ and $\mathcal{D}_{3}$ to the classical constitutive relations must be $\Theta$-preserving (same as expected for the respective operators in table 1), while that from $\mathcal{D}_{2}$ must be $\Theta-$ violating (opposite to expected for respective operators). Noting that $\mathcal{D}_{3}$ only contributes to the constitutive relations at two-derivative order and higher, these conditions imply, for instance, that one-derivative dissipative transport (produces entropy) must be $\Theta$-violating, while one-derivative adiabatic transport (does not produce entropy) must be $\Theta$-preserving. This should be physically expected for a dissipative system. In linear hydrodynamics, these requirements implement Onsager's reciprocity relations.

\subsection{Field redefinitions and frame transformations}

The dynamical fields in the effective field theory can, in general, be subjected to arbitrary field redefinitions. However, having chosen the fields to realise a particular representation of the KMS transformations in eq. (3.26) fixes a large part of this freedom. For concreteness, let us work in the physical spacetime representation; we also restrict to the statistical limit for simplicity. We consider a general transformation of the " $a$ " type fields

$$
X_{a}^{\mathrm{M}} \rightarrow X_{a}^{\mathrm{M}}+i f_{a}^{\mathrm{M}},
$$

where $f_{a}^{\mathrm{M}}$ can be arbitrary symmetry-respecting functions of the building blocks $G_{r, a \mathrm{MN}}$, $V^{\mathrm{M}}$, and $\beta^{\mathrm{M}}$. Due to the condition (3.25b), these quantities must be at least linear in $G_{a \mathrm{MN}}$, and due to eq. (3.25a), each occurrence of $G_{a \mathrm{MN}}$ must be accompanied with a factor of $i$. Finally, to respect the KMS transformations (3.26), the hydrodynamic fields $\beta^{\mathrm{M}}$ must transform as

$$
\beta^{\mathrm{M}} \rightarrow \beta^{\mathrm{M}}+\left(f_{a}^{\mathrm{M}}-\Theta \tilde{f}_{a}^{\mathrm{M}}\right)+\mathcal{O}(\hbar) .
$$

Interestingly, we find that the redefinitions of $\beta^{\mathrm{M}}$ are entirely constrained by the redefinitions of $X_{a}^{\mathrm{M}}$. This, in turn, constrains the redefinitions in the remaining dynamical fields $\sigma^{\mathrm{A}}$ through the definitions (3.10). Note that the KMS "tilde" conjugation of $G_{r \mathrm{MN}}, V^{\mathrm{M}}$, and $\beta^{\mathrm{M}}$ in the statistical limit in eq. (3.26) is same as a $\Theta$-conjugation, while that of $G_{a \mathrm{MN}}$ is same as a $\Theta$-conjugation up to terms involving $£_{\beta} G_{r \mathrm{MN}}$. Hence, KMS conjugation of $f_{a}^{\mathrm{M}}$ followed by $\Theta$-conjugation yields back the original quantities up to terms involving $£_{\beta} G_{r \mathrm{MN}}$. It follows that the allowed shifts of $\beta^{\mathrm{M}}$ in eq. (3.35b) are purely non-hydrostatic, i.e they involve at least one instance of $£_{\beta} G_{r \mathrm{MN}}$. In fact, since the constitutive relations following from the most generic KMS-respecting effective Lagrangian (3.29) always satisfy the adiabaticity equation (3.33), these allowed redefinitions merely correspond to the residual field redefinitions among thermodynamic frames; see section 2.3. 
During our discussion of classical Galilean hydrodynamics in section 2.4, we fixed the residual redefinition freedom among thermodynamic frames by eliminating any dependence on $u^{\mathrm{M}} \delta_{\mathscr{B}} g_{\mathrm{MN}}$ from the constitutive relations using the classical equations of motion. We can make an equivalent statement in the field theory as well. Note that any infinitesimal redefinitions of $X_{a}^{\mathrm{M}}$ change the Lagrangian by terms involving equations of motion. Hence, we can entirely fix the redefinitions $(3.35)$ by choosing to skip terms involving $u^{\mathrm{M}} \delta_{\mathscr{B}} G_{r \mathrm{MN}}$ while constructing the effective Lagrangian (3.29), which can always be eliminated using equations of motion. This is the analogue of working in the thermodynamic mass frame from classical hydrodynamics. Note that $G_{r \mathrm{MN}}$ are identified with the average sources $g_{\mathrm{MN}}$ in the statistical limit.

This concludes our formal discussion of the Schwinger-Keldysh effective field theory framework for Galilean hydrodynamics. We outlined a set of effective fields and rules that must be respected by an effective action describing Galilean hydrodynamics. We then proceeded to apply these rules in small $\hbar$ limit to construct the most generic effective theory governing stochastic fluctuations in a Galilean fluid. We also illustrated how the classical local second law of thermodynamics and Onsager's relations emerge within the field theory.

\section{Effective action for one-derivative Galilean fluids}

Our discussion of the EFT framework for Galilean hydrodynamics so far has been quite formal. As a concrete realisation of these ideas, we now write down the explicit effective action describing one-derivative Galilean fluids from section 2, using the generic machinery from section 3.4. We then proceed to linearise this effective action around a fluid configuration at rest and isolate an interacting perturbative field theory describing stochastic fluctuations.

\subsection{Non-linear effective action}

Truncation of the EFT at a given derivative order requires us to pick a derivative counting scheme for various background and dynamical fields, based on the physical system under consideration. A natural choice is to treat the fluctuations of the hydrodynamic fields $\beta^{\mathrm{M}}$ (or equivalently of the conjugate conserved densities $T_{r}^{t \mathrm{M}}$ ) and noise fields $X_{a}^{\mathrm{M}}$ at the same order, taken to be $\mathcal{O}\left(\partial^{0}\right)$ for reference. It follows that $G_{a \mathrm{MN}}$ and $£_{\beta} G_{r \mathrm{MN}}$ should be treated as $\mathcal{O}\left(\partial^{1}\right)$. For consistency, we must choose the background fields $g_{r \mathrm{MN}}$ as $\mathcal{O}\left(\partial^{0}\right)$ and $g_{a \mathrm{MN}}$ as $\mathcal{O}\left(\partial^{1}\right)$. This counting scheme ensures that the KMS constraints in the statistical limit do not mix between derivative orders. Since the physical energy-momentum tensor is given by a variational derivative of the action with respect to $g_{a \mathrm{MN}}$, the "derivative order" of the hydrodynamic constitutive relations, or simply that of hydrodynamics, is given by one less than the order of the Lagrangian. It follows that the $\mathcal{D}_{m}$ operators in eq. (3.29) start contributing to the constitutive relations at $\mathcal{O}\left(\partial^{m-1}\right)$ in the derivative expansion.

Therefore, one-derivative hydrodynamics is entirely characterised by the most generic expressions for the operators $\mathcal{D}_{1}(\circ)$ and $\mathcal{D}_{2}(\circ, \circ)$ written in terms of the metric $G_{r \mathrm{MN}}=g_{r \mathrm{MN}}$, null isometry $V^{\mathrm{M}}$, and the thermal vector $\beta^{\mathrm{M}}$, satisfying the constraints (3.30). The operator $\mathcal{D}_{1}$ needs to involve all the allowed terms up to one derivative order, while $\mathcal{D}_{2}$ only needs to 
involve zero derivative terms. Let us start with $\mathcal{D}_{1}$. Focusing on $d=3$ spatial dimensions for parity-violating terms, while generic $d$ for parity-even terms, we propose that it takes the form

$$
\begin{aligned}
\mathcal{D}_{1}(G)=[ & \rho u^{\mathrm{M}} u^{\mathrm{N}}+2 \varepsilon u^{(\mathrm{M}} V^{\mathrm{N})}+p \Delta^{\mathrm{MN}} \\
& +2 u^{(\mathrm{M}}\left(a_{0} \epsilon^{\mathrm{N}) \mathrm{PRST}} V_{\mathrm{P}} u_{\mathrm{R}} \partial_{\mathrm{S}} u_{\mathrm{T}}+a_{2} \epsilon^{\mathrm{N}) \mathrm{PRST}} V_{\mathrm{P}} u_{\mathrm{R}} \partial_{\mathrm{S}} V_{\mathrm{T}}\right) \\
& \left.+2 V^{(\mathrm{M}}\left(a_{2} \epsilon^{\mathrm{N}) \mathrm{PRST}} V_{\mathrm{P}} u_{\mathrm{R}} \partial_{\mathrm{S}} u_{\mathrm{T}}+a_{1} \epsilon^{\mathrm{N}) \mathrm{PRST}} V_{\mathrm{P}} u_{\mathrm{R}} \partial_{\mathrm{S}} V_{\mathrm{T}}\right)\right] G_{\mathrm{MN}},
\end{aligned}
$$

for arbitrary symmetric tensor $G_{\mathrm{MN}}$. Various quantities appearing here are the same as introduced in section 2. The fluid velocity $u^{\mathrm{M}}$, temperature $T$, and mass chemical potential $\mu$ are defined in terms of the field theoretic structures in eq. (3.11), while $\Delta^{\mathrm{MN}}=G_{r}^{\mathrm{MN}}+$ $2 u^{(\mathrm{M}} V^{\mathrm{N})}$. The mass density $\rho(T, \mu)$ and internal energy density $\varepsilon(T, \mu)$ are fixed in terms of the pressure $p(T, \mu)$ using the thermodynamic relations (2.12), while the parity-violating coefficients $a_{0}(T, \mu), a_{1}(T, \mu)$, and $a_{2}(T, \mu)$ are fixed in terms of three constants given in eq. (2.30). With these in place, $\mathcal{D}_{1}$ is the most generic operator truncated at onederivative order that satisfies the condition (3.30a), with $\mathcal{N}_{0}^{\mathrm{M}}=p \beta^{\mathrm{M}}+\Upsilon^{\mathrm{M}}$ where $\Upsilon^{\mathrm{M}}$ is given in eq. (2.29). To implement the condition (3.30b) we need to pick a $\Theta$ operator. Choosing $\Theta$ to be just the time-reversal operator $\mathrm{T}$ does not lead to any constraints. If we were to choose $\Theta$ to be PT, instead, the parity-violating terms above will no longer be allowed. Moving on, for the $\mathcal{D}_{2}$ operator we find

$$
\left.\mathcal{D}_{2}\left(G, G^{\prime}\right)=k_{\mathrm{B}} T\left[2 \eta \Delta^{\mathrm{M}(\mathrm{R}} \Delta^{\mathrm{s}) \mathrm{N}}+\left(\zeta-\frac{2}{d} \eta\right) \Delta^{\mathrm{MN}} \Delta^{\mathrm{RS}}+4 T \kappa V^{(\mathrm{M}} \Delta^{\mathrm{N})(\mathrm{R}} V^{\mathrm{s}}\right)\right] G_{\mathrm{MN}} G_{\mathrm{MN}}^{\prime},
$$

for generic tensors $G_{\mathrm{MN}}$ and $G_{\mathrm{MN}}^{\prime}$. Note that $\mathcal{D}_{2}$ is symmetric under the exchange of its arguments. We have chosen not to write down any terms along the vector $u^{\mathrm{M}}$, because the respective contribution will couple to $u^{\mathrm{M}} \delta_{\mathscr{B}} G_{r \mathrm{MN}}$ in the effective action which we have chosen to eliminate using the classical equations of motion; see section 3.5. All the terms in $\mathcal{D}_{2}$ respect (3.30b) for any choice of $\Theta$. On the other hand, the dissipative transport coefficients: shear viscosity $\eta(T, \mu)$, bulk viscosity $\zeta(T, \mu)$, and thermal conductivity $\kappa(T, \mu)$ are constrained to be non-negative due to the condition (3.30c).

Plugging the operators (4.2) into eq. (3.29), we can explicitly work out the effective action for one-derivative Galilean hydrodynamics in the statistical limit

$$
\begin{aligned}
\mathcal{L}=\frac{1}{2} & \left(\rho u^{\mathrm{M}} u^{\mathrm{N}}+2 \varepsilon u^{(\mathrm{M}} V^{\mathrm{N})}+p \Delta^{\mathrm{MN}}\right)\left(g_{a \mathrm{MN}}+£_{X_{a}} g_{r \mathrm{MN}}\right) \\
& +\left(a_{0} \epsilon^{\mathrm{NPRST}} V_{\mathrm{P}} u_{\mathrm{R}} \partial_{\mathrm{S}} u_{\mathrm{T}}+a_{2} \epsilon^{\mathrm{NPRST}} V_{\mathrm{P}} u_{\mathrm{R}} \partial_{\mathrm{S}} V_{\mathrm{T}}\right)\left(u^{\mathrm{M}} g_{a \mathrm{MN}}+u^{\mathrm{M}} £_{X_{a}} g_{r \mathrm{MN}}\right) \\
& +\left(a_{2} \epsilon^{\mathrm{NPRST}} V_{\mathrm{P}} u_{\mathrm{R}} \partial_{\mathrm{S}} u_{\mathrm{T}}+a_{1} \epsilon^{\mathrm{NPRST}} V_{\mathrm{P}} u_{\mathrm{R}} \partial_{\mathrm{S}} V_{\mathrm{T}}\right)\left(V^{\mathrm{M}} g_{a \mathrm{MN}}+£_{X_{a}} V_{\mathrm{N}}\right) \\
& +\frac{i k_{\mathrm{B}} T}{4}\left(2 \eta \Delta^{\mathrm{M}(\mathrm{R}} \Delta^{\mathrm{S}) \mathrm{N}}+\left(\zeta-\frac{2}{d} \eta\right) \Delta^{\mathrm{MN}} \Delta^{\mathrm{RS}}\right)\left(g_{a \mathrm{MN}}+£_{X_{a}} g_{r \mathrm{MN}}\right)\left(g_{a \mathrm{RS}}+£_{\left(X_{a}+i \beta\right)} g_{r \mathrm{RS}}\right) \\
& +i k_{\mathrm{B}} T^{2} \kappa \Delta^{\mathrm{MR}}\left(g_{a \mathrm{MN}} V^{\mathrm{N}}+£_{X_{a}} V_{\mathrm{M}}\right)\left(g_{a \mathrm{RS}} V^{\mathrm{S}}+£_{\left(X_{a}+i \beta\right)} V_{\mathrm{R}}\right) \\
=\frac{1}{2} & T^{\mathrm{MN}}\left(g_{a \mathrm{MN}}+£_{X_{a}} g_{r \mathrm{MN}}\right) \\
& +\frac{i k_{\mathrm{B}} T}{4}\left(2 \eta \Delta^{\mathrm{M}(\mathrm{R}} \Delta^{\mathrm{S}) \mathrm{N}}+\left(\zeta-\frac{2}{d} \eta\right) \Delta^{\mathrm{MN}} \Delta^{\mathrm{RS}}\right)\left(g_{a \mathrm{MN}}+£_{X_{a}} g_{r \mathrm{MN}}\right)\left(g_{a \mathrm{RS}}+£_{X_{a}} g_{r \mathrm{RS}}\right) \\
& +i k_{\mathrm{B}} T^{2} \kappa \Delta^{\mathrm{MR}}\left(g_{a \mathrm{MN}} V^{\mathrm{N}}+£_{X_{a}} V_{\mathrm{M}}\right)\left(g_{a \mathrm{RS}} V^{\mathrm{S}}+£_{X_{a}} V_{\mathrm{R}}\right)
\end{aligned}
$$


where we have expanded the definitions of $G_{r \mathrm{MN}}$ and $G_{a \mathrm{MN}}$ in the statistical limit using eq. (3.8). Here $£_{X_{a}}$ denotes a Lie derivative along $X_{a}^{\mathrm{M}}$ and $£_{\left(X_{a}+i \beta\right)}$ along $X_{a}^{\mathrm{M}}+i \beta^{\mathrm{M}}$. In the second equality, we have introduced the classical constitutive relations $T^{\mathrm{MN}}$ in the thermodynamic mass frame given in eqs. (2.32) and (2.36). Varying the action with respect to $g_{a \mathrm{MN}}$, in a configuration with $X_{a}^{\mathrm{M}}=g_{a \mathrm{MN}}=0$, leads to the classical constitutive relations in the thermodynamic mass frame, given in eqs. (2.32) and (2.36), while extremising with respect to $X_{a}^{\mathrm{M}}$ leads to the associated classical conservation equations (2.22). The respective expressions in the Newton-Cartan language are presented in section 5.

Turning off the background fields, i.e. setting $g_{r \mathrm{MN}}=\eta_{\mathrm{MN}}, g_{a \mathrm{MN}}=0$, choosing the coordinates $\left(x^{\mathrm{M}}\right)=\left(x^{-}, t, x^{i}\right)$ such that $V^{\mathrm{M}}=\delta_{-}^{\mathrm{M}}$, and identifying $\varphi_{a}=-X_{a}^{-}$, the effective action (4.3) results in

$$
\begin{aligned}
\mathcal{L}=\rho & \left(\partial_{t}+u^{i} \partial_{i}\right) \varphi_{a}-\left(\varepsilon+\frac{1}{2} \rho \vec{u}^{2}+a_{0} \epsilon^{i j k} u_{i} \partial_{j} u_{k}\right)\left(\partial_{t}+u^{i} \partial_{i}\right) X_{a}^{t} \\
& +\left(\rho u^{i}+a_{0} \epsilon^{i j k} \partial_{j} u_{k}\right)\left(\partial_{t}+u^{k} \partial_{k}\right) X_{a i}+p\left(\partial_{i} X_{a}^{i}-u^{i} \partial_{i} X_{a}^{t}\right) \\
& +a_{0} \epsilon^{i j k} \partial_{j} u_{k}\left(\partial_{i} \varphi_{a}+u^{l} \partial_{i} X_{a l}\right)-\left(a_{2}+a_{0} \frac{1}{2} \vec{u}^{2}\right) \epsilon^{i j k} \partial_{j} u_{k} \partial_{i} X_{a}^{t} \\
& +2 i k_{\mathrm{B}} T \eta\left(\partial_{(i} X_{a j}-u_{(i} \partial_{j} X_{a}^{t}\right)\left(\partial^{(i} X_{a}^{j)}-u^{(i} \partial^{j)} X_{a}^{t}+i / T \partial^{(i} u^{j)}\right) \\
& +i k_{\mathrm{B}} T\left(\zeta-\frac{2}{d} \eta\right)\left(\partial_{i} X_{a}^{i}-u^{i} \partial_{i} X_{a}^{t}\right)\left(\partial_{k} X_{a}^{k}-u^{k} \partial_{k} X_{a}^{t}+i / T \partial_{k} u^{k}\right) \\
& +i k_{\mathrm{B}} \kappa \partial_{i} X_{a}^{t}\left(T^{2} \partial^{i} X_{a}^{t}-i \partial^{i} T\right) \\
=\rho^{t} & \partial_{t} \varphi_{a}+\pi^{i} \partial_{i} \varphi_{a}-\epsilon^{t} \partial_{t} X_{a}^{t}-\epsilon^{i} \partial_{i} X_{a}^{t}+\pi^{i} \partial_{t} X_{a i}+\tau^{i j} \partial_{i} X_{a j} \\
& +2 i k_{\mathrm{B}} T \eta\left(\partial_{(i} X_{a j}-u_{(i} \partial_{j} X_{a}^{t}\right)\left(\partial^{(i} X_{a}^{j)}-u^{(i} \partial^{j)} X_{a}^{t}\right)+i k_{\mathrm{B}} T\left(\zeta-\frac{2}{d} \eta\right)\left(\partial_{k} X_{a}^{k}-u^{k} \partial_{k} X_{a}^{t}\right)^{2} \\
& +i k_{\mathrm{B}} T^{2} \kappa \partial_{i} X_{a}^{t} \partial^{i} X_{a}^{t} .
\end{aligned}
$$

Again, in the second step, we have substituted the thermodynamic mass frame constitutive relations from eq. (2.37). All the parity-violating terms get absorbed within the constitutive relations. Non-trivial contributions only arise due to the dissipative terms. This action is merely the generalisation of the effective action (1.5) presented in the introduction, to include parity-violating effects. Note that the effective action (4.4) is applicable at the full non-linear level; in the next subsection we inspect this in a linearised limit.

\subsection{Linearised stochastic fluctuations}

\subsubsection{Perturbative expansion in fluctuations}

Let us consider a Galilean fluid at rest coupled to a flat background. In the EFT terms, the fluid is described by the effective action (4.4), with the equilibrium state

$$
\tau=t, \quad \sigma^{i}=x^{i}, \quad \varphi_{r}=0, \quad X_{a}^{t}=X_{a}^{i}=\varphi_{a}=0 .
$$

The hydrodynamic fields in this state are given using eq. (3.10) as $\beta^{\mathrm{M}}=\delta_{\mathrm{A}}^{\mathrm{M}} \beta^{\mathrm{A}}$. We can choose the thermal reference vector to be $\beta^{\mathrm{A}}=\beta_{0}\left(\delta_{t}^{\mathrm{M}}-\mu_{0} \delta_{-}^{\mathrm{M}}\right)$, which implies that $T=T_{0}, \mu=$ $\mu_{0}$, and $u^{i}=0$ in equilibrium. We would like to expand the effective action perturbatively in 
fluctuations around the equilibrium state. In the " $a$ " sector we can work with the fields $X_{a}^{t}$, $X_{a}^{i}, \varphi_{a}$ directly, while in the " $r$ " sector it is instead convenient to work with the fluctuations in the physical conserved densities: $\delta \rho=\rho^{t}-\rho_{0}, \delta \epsilon=\epsilon^{t}-\varepsilon_{0}, \pi^{i}$ given in eq. (2.37). These are related to fluctuations of hydrodynamic variables $\delta T=T-T_{0}, \delta \mu=\mu-\mu_{0}$, and $u^{i}$ through

$$
\begin{aligned}
u^{i}= & \frac{1}{\rho_{0}} \pi^{i}-\frac{2 K_{0} T_{0}}{\rho_{0}^{2}} \epsilon^{i j k} \partial_{j} \pi_{k}-\frac{1}{\rho_{0}^{2}} \pi^{i} \delta \rho \\
& -\frac{2 K_{0} T_{0}}{\rho_{0}^{3}} \epsilon^{i j k} \pi_{j} \partial_{k} \delta \rho-\left(\frac{\partial}{\partial \rho} \frac{2 K_{0} T}{\rho^{2}}\right)_{0} \epsilon^{i j k} \partial_{j} \pi_{k} \delta \rho-\left(\frac{2 K_{0}}{\rho^{2}} \frac{\partial T}{\partial \varepsilon}\right)_{0} \epsilon^{i j k} \partial_{j} \pi_{k} \delta \epsilon+\ldots \\
\rho(T, \mu)= & \rho_{0}+\delta \rho, \quad \varepsilon(T, \mu)=\varepsilon_{0}+\delta \epsilon-\frac{1}{2 \rho_{0}} \vec{\pi}^{2}+\ldots
\end{aligned}
$$

These expressions are valid until quadratic order in fields and leading order in derivatives. The subscript "0" on various coefficients represents evaluation on the equilibrium configuration $T=T_{0}, \mu=\mu_{0}$. The thermodynamic derivatives have been performed in the microcanonical ensemble controlled by $(\varepsilon, \rho)$. On the other hand, the hydrodynamic fields are related to $\delta \sigma^{\tau}=\sigma^{\tau}-t, \delta \sigma^{i}=\sigma^{i}-x^{i}$, and $\varphi_{r}$ through eq. (3.11) leading to

$$
\begin{aligned}
& \delta T=T_{0} \partial_{t} \delta \sigma^{\tau}-T_{0} \partial_{t} \delta \sigma^{k} \partial_{k} \delta \sigma^{\tau}+\ldots, \\
& \delta \mu=\partial_{t}\left(\varphi_{r}+\mu_{0} \delta \sigma^{\tau}\right)-\partial_{t} \delta \sigma^{k} \partial_{k}\left(\varphi_{r}+\mu_{0} \delta \sigma^{\tau}\right)+\ldots
\end{aligned} \quad u^{i}=-\partial_{t} \delta \sigma^{i}+\partial_{t} \delta \sigma^{k} \partial_{k} \delta \sigma^{i} \ldots
$$

We can substitute eq. (4.6) into the constitutive relations for $\epsilon^{i}$ and $\tau^{i j}$ in eq. (2.37) and truncate the expressions to leading order in derivatives and quadratic order in fluctuations to find $^{17}$

$$
\begin{aligned}
& \epsilon^{i}=\frac{\varepsilon_{0}+p_{0}}{\rho_{0}} \pi^{i}-\left(\kappa \frac{\partial T}{\partial \rho}\right)_{0} \partial^{i} \rho-\left(\kappa \frac{\partial T}{\partial \varepsilon}\right)_{0} \partial^{i} \varepsilon+\left(\frac{\xi_{\Omega}}{\rho}\right)_{0} \epsilon^{i j k} \partial_{j} \pi_{k} \\
& +\frac{1}{\rho_{0}}\left(1+\frac{\partial p}{\partial \varepsilon}\right)_{0} \pi^{i} \delta \epsilon+\frac{1}{\rho_{0}}\left(\frac{\partial p}{\partial \rho}-\frac{\epsilon+p}{\rho}\right)_{0} \pi^{i} \delta \rho \\
& -\left[\frac{\partial}{\partial \rho}\left(\kappa \frac{\partial T}{\partial \rho}\right)\right]_{0} \delta \rho \partial^{i} \rho-\left[\frac{\partial}{\partial \varepsilon}\left(\kappa \frac{\partial T}{\partial \rho}\right)\right]_{0} \delta \varepsilon \partial^{i} \rho \\
& -\left[\frac{\partial}{\partial \rho}\left(\kappa \frac{\partial T}{\partial \varepsilon}\right)\right]_{0} \delta \rho \partial^{i} \delta \varepsilon-\left[\frac{\partial}{\partial \varepsilon}\left(\kappa \frac{\partial T}{\partial \varepsilon}\right)\right]_{0} \delta \varepsilon \partial^{i} \delta \varepsilon \\
& +\frac{1}{2}\left(\frac{\kappa}{\rho} \frac{\partial T}{\partial \varepsilon}\right)_{0} \partial^{i} \vec{\pi}^{2}-\frac{\eta_{0}}{\rho_{0}^{2}}\left(\frac{1}{2} \partial^{i} \vec{\pi}^{2}+\pi^{k} \partial_{k} \pi^{i}\right)-\frac{\zeta_{0}-\frac{2}{d} \eta_{0}}{\rho_{0}^{2}} \pi^{i} \partial_{k} \pi^{k} \\
& +\left(\frac{\partial}{\partial \rho} \frac{\xi_{\Omega}}{\rho}\right)_{0} \epsilon^{i j k} \partial_{j} \pi_{k} \delta \rho+\left(\frac{\partial}{\partial \varepsilon} \frac{\xi_{\Omega}}{\rho}\right)_{0} \epsilon^{i j k} \partial_{j} \pi_{k} \delta \epsilon+\left(\frac{\xi_{\Omega}}{\rho^{2}}\right)_{0} \epsilon^{i j k} \pi_{j} \partial_{k} \delta \rho+\ldots, \\
& \tau^{i j}=p_{0} \delta^{i j}+\left(\frac{\partial p}{\partial \rho}\right)_{0} \delta^{i j} \delta \rho+\left(\frac{\partial p}{\partial \varepsilon}\right)_{0} \delta^{i j} \delta \epsilon-\frac{\eta_{0}}{\rho_{0}} 2 \partial^{(i} \pi^{j)}-\frac{\zeta_{0}-\frac{2}{d} \eta_{0}}{\rho_{0}} \delta^{i j} \partial_{k} \pi^{k} \\
& +\frac{1}{\rho_{0}} \pi^{i} \pi^{j}-\frac{1}{2 \rho_{0}}\left(\frac{\partial p}{\partial \varepsilon}\right)_{0} \delta^{i j} \vec{\pi}^{2}+\frac{1}{2}\left(\frac{\partial^{2} p}{\partial \rho^{2}}\right)_{0} \delta^{i j} \delta \rho^{2}+\left(\frac{\partial^{2} p}{\partial \rho \delta \varepsilon}\right)_{0} \delta^{i j} \delta \rho \delta \epsilon+\frac{1}{2}\left(\frac{\partial^{2} p}{\partial \varepsilon^{2}}\right)_{0} \delta^{i j} \delta \epsilon^{2} \\
& -\left(\frac{\partial}{\partial \rho} \frac{\eta}{\rho}\right)_{0} 2 \partial^{(i} \pi^{j)} \delta \rho-\left(\frac{\partial}{\partial \varepsilon} \frac{\eta}{\rho}\right)_{0} 2 \partial^{(i} \pi^{j)} \delta \epsilon+\frac{\eta_{0}}{\rho_{0}^{2}} 2 \pi^{(i} \partial^{j)} \delta \rho \\
& -\left(\frac{\partial}{\partial \rho} \frac{\zeta-\frac{2}{d} \eta}{\rho}\right)_{0} \delta^{i j} \partial_{k} \pi^{k} \delta \rho-\left(\frac{\partial}{\partial \varepsilon} \frac{\zeta-\frac{2}{d} \eta}{\rho}\right)_{0} \delta^{i j} \partial_{k} \pi^{k} \delta \epsilon+\frac{\zeta_{0}-\frac{2}{d} \eta_{0}}{\rho_{0}^{2}} \delta^{i j} \pi^{k} \partial_{k} \delta \rho+\ldots
\end{aligned}
$$

\footnotetext{
${ }^{17}$ Tip: it is actually easier to start with the mass frame constitutive relations, where $u^{i}$ is just $\pi^{i} / \rho^{t}$.
} 
The parity-odd transport coefficient $\xi_{\Omega}$ has been defined in eq. (2.30). If required, this expansion can be extended to higher orders in fluctuations.

\subsubsection{Free theory}

We can substitute the expansion of constitutive relations from eq. (4.8) into eq. (4.4) and work out the hydrodynamic effective action order-by-order in fluctuations. Note that the effective action is always one order higher in derivatives and fluctuations compared to the constitutive relations. For example, truncated to quadratic order in fields, we find the "free" Lagrangian

$$
\begin{aligned}
\mathcal{L}_{\text {free }}= & -\varphi_{a}\left(\partial_{t} \delta \rho+\partial_{i} \pi^{i}\right)+X_{a}^{t}\left(\partial_{t} \delta \epsilon-\kappa \frac{\partial T}{\partial \varepsilon} \partial^{i} \partial_{i} \delta \epsilon-\kappa \frac{\partial T}{\partial \rho} \partial^{i} \partial_{i} \delta \rho\right)+\frac{\varepsilon+p}{\rho} X_{a}^{t} \partial_{i} \pi^{i} \\
& -X_{a}^{i}\left(\partial_{t} \pi_{i}+\frac{\partial p}{\partial \rho} \partial_{i} \delta \rho+\frac{\partial p}{\partial \varepsilon} \partial_{i} \delta \epsilon-\frac{\eta}{\rho} \partial^{k} \partial_{k} \pi_{i}-\frac{\zeta+\frac{d-2}{d} \eta}{\rho} \partial_{j} \partial_{i} \pi^{i}\right) \\
& +i k_{\mathrm{B}} T^{2} \kappa \partial^{i} X_{a}^{t} \partial_{i} X_{a}^{t}+i k_{\mathrm{B}} T \eta \partial_{i} X_{a j} \partial^{i} X_{a}^{j}+i k_{\mathrm{B}} T\left(\zeta+\frac{d-2}{d} \eta\right)\left(\partial_{k} X_{a}^{k}\right)^{2}
\end{aligned}
$$

where we have ignored certain total derivative terms. We have also dropped the subscript "0" from the coefficients for clarity. We can write this out in momentum space

$$
\begin{aligned}
& \mathcal{L}= \\
& \left(\begin{array}{c}
\varphi_{a}(-p) \\
-X_{a}^{t}(-p) \\
X_{a}^{j}(-p)
\end{array}\right)^{\mathrm{T}}\left(\begin{array}{ccc}
i \omega & 0 & -i k_{i} \\
-\kappa \partial T / \partial \rho k^{2} & i \omega-\kappa \partial T / \partial \varepsilon k^{2} & -(\varepsilon+p) / \rho i k_{i} \\
-\partial p / \partial \rho i k_{j} & -\partial p / \partial \varepsilon i k_{j} & i \omega \delta_{i j}-\eta / \rho \delta_{i j} k^{2}-\left(\zeta-\frac{2}{d} \eta\right) / \rho k_{i} k_{j}
\end{array}\right)\left(\begin{array}{c}
\delta \rho(p) \\
\delta \epsilon(p) \\
\pi^{i}(p)
\end{array}\right) \\
& +\frac{i}{2}\left(\begin{array}{c}
\varphi_{a}(-p) \\
-X_{a}^{t}(-p) \\
X_{a}^{j}(-p)
\end{array}\right)^{\mathrm{T}}\left(\begin{array}{ccc}
0 & 0 & 0 \\
0 & 2 k_{\mathrm{B}} T^{2} \kappa k^{2} & 0 \\
0 & 0 & 2 k_{\mathrm{B}} T \eta \delta_{i j} k^{2}+2 k_{\mathrm{B}} T\left(\zeta-\frac{2}{d} \eta\right) k_{i} k_{j}
\end{array}\right)\left(\begin{array}{c}
\varphi_{a}(p) \\
-X_{a}^{t}(p) \\
X_{a}^{i}(p)
\end{array}\right) \\
& =\varphi_{a}^{I}(-p) K_{I}^{J}(p) \mathcal{O}_{J}(p)+\frac{i}{2} \varphi_{a}^{I}(-p) G_{I J} \varphi_{a}^{J}(p) .
\end{aligned}
$$

Here $p=(\omega, k)$ in the arguments collectively denotes frequency and momentum. In the second step, we have collectively denoted the fluctuations in conserved operators by $\mathcal{O}_{I}=$ $\left(\delta \rho, \delta \epsilon, \pi_{i}\right)$ and the auxiliary fields by $\varphi_{a}^{I}=\left(\varphi_{a},-X_{a}^{t}, X_{a}^{i}\right)$. From here one can read out the momentum-space free propagators

$$
\begin{aligned}
\left\langle\mathcal{O}_{I}(p) \varphi_{a}^{J}(-p)\right\rangle_{0} & =i\left(K^{-1}\right)_{I}^{J}(p), & & \left\langle\varphi_{a}^{I}(p) \mathcal{O}_{J}(-p)\right\rangle_{0}=i\left(K^{-1 *}\right)_{J}^{I}(p), \\
\left\langle\mathcal{O}_{I}(p) \mathcal{O}_{J}(-p)\right\rangle_{0} & =\left(K^{-1} G K^{-\mathrm{T} *}\right)_{I J}(p), & & \left\langle\varphi_{a}^{I}(p) \varphi_{a}^{J}(-p)\right\rangle_{0}=0 .
\end{aligned}
$$

The poles of the propagators are controlled by $\operatorname{det} K$. As expected, we find a total of $(d+2)$ modes: a pair of sound mode, a longitudinal charge diffusion mode, and $d-1$ copies of transverse shear diffusion mode. In small momentum limit, they are given by

$$
\omega= \pm v_{s} k-\frac{i}{2} \Gamma_{s} k^{2}, \quad \omega=-i D_{\|} k^{2}, \quad \omega=-i D_{\perp} k^{2}
$$


where the speed of sound, attenuation constant, and diffusion constants are respectively given as $^{18}$

$$
\begin{aligned}
v_{s}^{2} & =\frac{\partial p}{\partial \rho}+\frac{\varepsilon+p}{\rho} \frac{\partial p}{\partial \varepsilon}, & \Gamma_{s} & =\frac{\zeta+2 \frac{d-1}{d} \eta}{\rho}+\frac{T \kappa}{v_{s}^{2} \rho}\left(\frac{\partial p}{\partial \varepsilon}\right)^{2} \\
D_{\|} & =\frac{\kappa}{v_{s}^{2}}\left(\frac{\partial p}{\partial \rho} \frac{\partial T}{\partial \varepsilon}-\frac{\partial p}{\partial \varepsilon} \frac{\partial T}{\partial \rho}\right) & D_{\perp} & =\frac{\eta}{\rho} .
\end{aligned}
$$

One can see that the propagators $\left\langle\mathcal{O}_{I} \varphi_{a}^{J}\right\rangle_{0}$ are purely retarded, while $\left\langle\varphi_{a}^{I} \mathcal{O}_{J}\right\rangle_{0}$ are purely advanced. Coupling the theory to background sources, one can indeed verify that they are related to retarded and advanced propagators of the hydrodynamic densities $\mathcal{O}_{I}$ respectively, while $\left\langle\mathcal{O}_{I} \mathcal{O}_{J}\right\rangle$ are the symmetric propagators. To wit

$$
\begin{aligned}
& G_{\mathcal{O}_{I} \mathcal{O}_{J}}^{\mathrm{R}, 0}(p)=-i \omega\left(K^{-1}\right)_{I}^{K}(p) \chi_{K J}, \quad G_{\mathcal{O}_{I} \mathcal{O}_{J}}^{\mathrm{A}, 0}(p)=i \omega\left(K^{-1 *}\right)_{J}^{K}(p) \chi_{K I}, \\
& G_{\mathcal{O}_{I} \mathcal{O}_{J}}^{\mathrm{S}, 0}(p)=\left(K^{-1} G K^{-\mathrm{T} *}\right)_{I J}(p),
\end{aligned}
$$

up to contact terms. Here $\chi_{I J}$ is the susceptibility matrix

$$
\chi_{I J}=\left(\begin{array}{ccc}
\partial \rho / \partial \mu & \partial \varepsilon / \partial \mu & 0 \\
\partial \varepsilon / \partial \mu & T \partial \epsilon / \partial T+\mu \partial \epsilon / \partial \mu & 0 \\
0 & 0 & \rho
\end{array}\right)=\left(\begin{array}{ccc}
T \partial(\mu / T) / \partial \rho & 1 / T \partial T / \partial \rho & 0 \\
1 / T \partial T / \partial \rho & 1 / T \partial T / \partial \varepsilon & 0 \\
0 & 0 & 1 / \rho
\end{array}\right)^{-1}
$$

It can be checked that the retarded function satisfies the Onsager's relations: $G_{\mathcal{O}_{I} \mathcal{O}_{J}}^{\mathrm{R}, 0}=$ $G_{\mathcal{O}_{J} \mathcal{O}_{I}}^{\mathrm{R}, 0}$. In addition, the three correlations functions are related by

$$
G_{\mathcal{O}_{I} \mathcal{O}_{J}}^{\mathrm{A}, 0}=\left(G_{\mathcal{O}_{I} \mathcal{O}_{J}}^{\mathrm{R}, 0}\right)^{*}, \quad G_{\mathcal{O}_{I} \mathcal{O}_{J}}^{\mathrm{S}, 0}=\frac{2 k_{\mathrm{B}} T}{\omega} \operatorname{Im} G_{\mathcal{O}_{I} \mathcal{O}_{J}}^{\mathrm{R}, 0}
$$

The second of these is the well-known two-point fluctuation-dissipation theorem.

\subsubsection{Interactions}

To account for interactions between hydrodynamic and stochastic degrees of freedom, we need to expand the effective action to higher order in fluctuations. Substituting eq. (4.8) into eq. (4.4), we find leading order three-point interaction terms coming from ideal hydrodynamics

$$
\mathcal{L}_{\mathrm{int}}^{1 \mathrm{der}}=\frac{1}{\rho} \pi^{i} \pi^{j} \partial_{i} X_{a j}-\left(\alpha_{\rho} \delta \rho+\alpha_{\epsilon} \delta \epsilon\right) \pi^{i} \partial_{i} X_{a}^{t}+\left(\frac{1}{2} \beta_{\rho} \delta \rho^{2}+\frac{1}{2} \beta_{\epsilon} \delta \epsilon^{2}+\beta_{\rho \epsilon} \delta \epsilon \delta \rho+\frac{1}{2} \beta_{\pi} \vec{\pi}^{2}\right) \partial_{i} X_{a}^{i},
$$

where we have defined the couplings

$$
\begin{aligned}
& \alpha_{\rho}=\frac{1}{\rho}\left(\frac{\partial p}{\partial \rho}-\frac{\varepsilon+p}{\rho}\right), \quad \alpha_{\epsilon}=\frac{1}{\rho}\left(1+\frac{\partial p}{\partial \varepsilon}\right), \\
& \beta_{\rho}=\frac{\partial^{2} p}{\partial \rho^{2}}, \quad \beta_{\epsilon}=\frac{\partial^{2} p}{\partial \varepsilon^{2}}, \quad \beta_{\rho \epsilon}=\frac{\partial^{2} p}{\partial \rho \partial \varepsilon}, \quad \beta_{\pi}=-\frac{1}{\rho} \frac{\partial p}{\partial \varepsilon} .
\end{aligned}
$$

\footnotetext{
${ }^{18} \frac{\partial T}{\partial \rho}+\frac{\varepsilon+p}{\rho} \frac{\partial T}{\partial \varepsilon}=\frac{T}{\rho} \frac{\partial p}{\partial \varepsilon}$.
} 
Similarly, we can work out two-derivative three-point interactions due to one-derivative corrections to the constitutive relations. We have unitary interactions involving two hydrodynamic and one stochastic fields like above

$$
\begin{aligned}
\mathcal{L}_{\text {int }}^{2 \mathrm{der}, \mathrm{rra}}= & \frac{\eta}{\rho^{2}} \pi^{i} \partial_{i} \pi^{j} \partial_{j} X_{a}^{t}-\frac{\eta}{\rho^{2}} \delta \rho \pi_{i} \partial^{k} \partial_{k} X_{a}^{i}-\left(\theta_{\rho}^{\eta} \delta \rho+\theta_{\epsilon}^{\eta} \delta \epsilon\right)\left(\partial_{j} \pi_{i} \partial^{i} X_{a}^{j}+\partial_{i} \pi_{j} \partial^{i} X_{a}^{j}\right) \\
& +\frac{\zeta-\frac{2}{d} \eta}{\rho^{2}} \partial_{k} \pi^{k} \pi^{i} \partial_{i} X_{a}^{t}-\frac{\zeta+\frac{d-2}{d} \eta}{\rho^{2}} \delta \rho \pi^{i} \partial_{i} \partial_{j} X_{a}^{j}-\left(\theta_{\rho}^{\zeta} \delta \rho+\theta_{\epsilon}^{\zeta} \delta \epsilon\right) \partial_{k} \pi^{k} \partial_{i} X_{a}^{i} \\
& -\left(\frac{1}{2} \lambda_{\rho} \delta \rho^{2}+\frac{1}{2} \lambda_{\epsilon} \delta \epsilon^{2}+\lambda_{\rho \epsilon} \delta \rho \delta \epsilon+\frac{1}{2} \lambda_{\pi} \vec{\pi}^{2}\right) \partial^{k} \partial_{k} X_{a}^{t}-\lambda_{\rho \epsilon}^{\prime}\left(\delta \rho \partial^{i} \delta \epsilon-\delta \epsilon \partial^{i} \delta \rho\right) \pi^{i} \partial_{i} X_{a}^{t} \\
& -\left(\theta_{\rho}^{\xi} \delta \rho+\theta_{\epsilon}^{\xi} \delta \epsilon\right) \epsilon^{i j k} \partial_{j} \pi_{k} \partial_{i} X_{a}^{t},
\end{aligned}
$$

with couplings

$$
\begin{aligned}
& \theta_{\rho}^{\eta}=\frac{1}{\rho} \frac{\partial \eta}{\partial \rho} \\
& \theta_{\epsilon}^{\eta}=\frac{1}{\rho} \frac{\partial \eta}{\partial \varepsilon} \\
& \theta_{\rho}^{\zeta}=\frac{1}{\rho} \frac{\partial\left(\zeta-\frac{2}{d} \eta\right)}{\partial \rho}, \quad \theta_{\epsilon}^{\zeta}=\frac{1}{\rho} \frac{\partial\left(\zeta-\frac{2}{d} \eta\right)}{\partial \varepsilon}, \\
& \lambda_{\rho}=\frac{\partial}{\partial \rho}\left(\kappa \frac{\partial T}{\partial \rho}\right) \\
& \lambda_{\epsilon}=\frac{\partial}{\partial \varepsilon}\left(\kappa \frac{\partial T}{\partial \varepsilon}\right), \quad \lambda_{\rho \epsilon}=\frac{1}{2}\left[\frac{\partial}{\partial \varepsilon}\left(\kappa \frac{\partial T}{\partial \rho}\right)+\frac{\partial}{\partial \rho}\left(\kappa \frac{\partial T}{\partial \varepsilon}\right)\right], \\
& \lambda_{\rho \epsilon}^{\prime}=\frac{1}{2}\left(\frac{\partial \kappa}{\partial \varepsilon} \frac{\partial T}{\partial \rho}-\frac{\partial \kappa}{\partial \rho} \frac{\partial T}{\partial \varepsilon}\right), \quad \lambda_{\pi}=\frac{1}{\rho}\left(\frac{\eta}{\rho}-\kappa \frac{\partial T}{\partial \varepsilon}\right), \quad \theta_{\rho}^{\xi}=\frac{1}{\rho} \frac{\partial \xi_{\Omega}}{\partial \rho}, \quad \theta_{\epsilon}^{\xi}=\frac{1}{\rho} \frac{\partial \xi_{\Omega}}{\partial \varepsilon} .
\end{aligned}
$$

Secondly, we have non-unitary terms involving one hydrodynamic and two stochastic fields

$$
\begin{aligned}
& \mathcal{L}_{\text {int }}^{2 \text { der,raa }}=i k_{\mathrm{B}} T^{2}\left(\frac{\tilde{\lambda}_{\rho}}{\partial T / \partial \rho} \delta \rho+\frac{\tilde{\lambda}_{\epsilon}}{\partial T / \partial \varepsilon} \delta \epsilon\right) \partial^{i} X_{a}^{t} \partial_{i} X_{a}^{t} \\
& \quad+i k_{\mathrm{B}} T \rho\left(\tilde{\theta}_{\rho}^{\eta} \delta \rho+\tilde{\theta}_{\epsilon}^{\eta} \delta \epsilon\right)\left(\partial_{i} X_{a j} \partial^{i} X_{a}^{j}+\partial_{i} X_{a j} \partial^{j} X_{a}^{i}\right)-\frac{2 i T k_{\mathrm{B}} \eta}{\rho}\left(\pi^{i} \partial^{j} X_{a}^{t}+\pi^{j} \partial^{i} X_{a}^{t}\right) \partial_{i} X_{a j} \\
& \quad+i k_{\mathrm{B}} T \rho\left(\tilde{\theta}_{\rho}^{\zeta} \delta \rho+\tilde{\theta}_{\epsilon}^{\zeta} \delta \epsilon\right)\left(\partial_{k} X_{a}^{k}\right)^{2}-\frac{2 i k_{\mathrm{B}} T\left(\zeta-\frac{2}{d} \eta\right)}{\rho} \pi^{k} \partial_{k} X_{a}^{t} \partial_{i} X_{a}^{i}
\end{aligned}
$$

with coefficients

$$
\begin{array}{lll}
\tilde{\theta}_{\rho}^{\eta}=\frac{1}{T \rho} \frac{\partial(T \eta)}{\partial \rho}, & \tilde{\theta}_{\epsilon}^{\eta}=\frac{1}{T \rho} \frac{\partial(T \eta)}{\partial \varepsilon}, & \tilde{\theta}_{\rho}^{\zeta}=\frac{1}{T \rho} \frac{\partial\left(T \zeta-\frac{2}{d} T \eta\right)}{\partial \rho}, \quad \tilde{\theta}_{\epsilon}^{\zeta}=\frac{1}{T \rho} \frac{\partial\left(T \zeta-\frac{2}{d} T \eta\right)}{\partial \varepsilon}, \\
\tilde{\lambda}_{\rho}=\frac{1}{T^{2}} \frac{\partial T}{\partial \rho} \frac{\partial\left(T^{2} \kappa\right)}{\partial \rho}, & \tilde{\lambda}_{\epsilon}=\frac{1}{T^{2}} \frac{\partial T}{\partial \varepsilon} \frac{\partial\left(T^{2} \kappa\right)}{\partial \varepsilon} .
\end{array}
$$

Note that not all the couplings appearing above are independent. In total, there can only be 15 couplings at this order in the parity even sector: pressure $p$, two thermodynamic first derivatives of $p$, and three second derivatives, three dissipative coefficients, and their 2 thermodynamic derivatives each. In the parity violating sector in $d=3$, there are just constants $K_{0}$ and $K_{2} ; K_{1}$ does not contribute in the absence of background fields. Most couplings appearing above are related due to the underlying Galilean symmetry of the effective theory. However, there are certain relations between unitary and non-unitary parts of the interaction Lagrangian; these can be understood as a realisation of three-point fluctuation dissipation theorem. If required, we can work out higher point interactions in a similar manner. 
Few comments are in order regarding the validity of the perturbative expansion itself, as we do not seem to have a small parameter controlling the strength of interactions. Since we are interested in a low-energy effective theory, we can treat momentum itself as the small parameter for the purposes of loop counting. In hydrodynamics, due to the presence of the sound mode, we typically take the frequency to scale as $\omega \sim k$ and $T_{0} \sim 1$. Since the Lagrangian must scale as $\mathcal{L} \sim \omega k^{d} \sim k^{d+1}$, eq. (4.9) implies that all the fields $\delta \rho, \delta \epsilon, \pi_{i}, \varphi_{a}$, $X_{a}^{t}, X_{a}^{i}$ scale as $k^{d / 2}$. As a consequence, all the leading derivative three-point couplings in eq. (4.17) are irrelevant by $k^{-d / 2}$ in the RG sense, while the subleading couplings in eqs. (4.19) and (4.21) are irrelevant by $k^{-d / 2-1}$. Similarly, all higher derivative and nonlinear couplings are further suppressed within the effective theory. Care should be taken in lower number of dimensions; in $d=2$ spatial dimensions the interaction terms in eq. (4.17) are as important as the dissipative corrections in the free Lagrangian (4.9), while in $d=1$ they become more important. The perturbative expansion is still valid in these dimensions, but the results are qualitatively different; see [23] for an example in the simpler case of energy diffusion model.

\subsection{Incompressible and diffusion limits}

As is evident from our discussion above, the full interacting theory of stochastic hydrodynamics is quite rich. For practical applications, therefore, it is often convenient to work in certain simplifying limits. Most popular of these is the incompressible limit where one ignores the density fluctuations $\delta \rho$. Inspecting the full non-linear effective action (4.4), we can infer that the field $\varphi_{a}$ does not appear in the interactions at all and merely becomes a Lagrange multiplier for freezing the longitudinal momentum modes $\partial_{i} \pi^{i}=0$. Correspondingly, we also choose $\partial_{i} X_{a}^{i}=0$. The resulting non-linear effective action is given as

$$
\begin{aligned}
\mathcal{L}^{\text {incom }}= & -\epsilon^{t} \partial_{t} X_{a}^{t}-\epsilon^{i} \partial_{i} X_{a}^{t}+\pi_{i} \partial_{t} X_{a i}+\tau^{i j} \partial_{i} X_{a j} \\
& +2 i k_{\mathrm{B}} T \eta\left(\partial_{(i} X_{a j}-u_{(i} \partial_{j} X_{a}^{t}\right)\left(\partial^{(i} X_{a}^{j)}-u^{(i} \partial^{j)} X_{a}^{t}\right)+i k_{\mathrm{B}} T^{2} \kappa \partial_{i} X_{a}^{t} \partial^{i} X_{a}^{t}
\end{aligned}
$$

We can apply these constraints to the linearised effective action in section 4.2 and obtain

$$
\begin{aligned}
\mathcal{L}_{\text {free }}^{\text {incom }}= & X_{a}^{t}\left(\partial_{t}-\kappa \frac{\partial T}{\partial \varepsilon} \partial^{i} \partial_{i}\right) \delta \epsilon+i k_{\mathrm{B}} T^{2} \kappa \partial^{i} X_{a}^{t} \partial_{i} X_{a}^{t}-X_{a}^{i}\left(\partial_{t}-\frac{\eta}{\rho} \partial^{k} \partial_{k}\right) \pi_{i} \\
& +i k_{\mathrm{B}} T \eta \partial_{i} X_{a j} \partial^{i} X_{a}^{j}, \\
\mathcal{L}_{\text {int }}^{\text {incom }}= & \frac{1}{\rho} \pi^{i} \pi^{j} \partial_{i} X_{a j}-\alpha_{\epsilon} \delta \epsilon \pi^{i} \partial_{i} X_{a}^{t}+\frac{\eta}{\rho^{2}} \pi^{i} \partial_{i} \pi^{j} \partial_{j} X_{a}^{t}-\theta_{\epsilon}^{\eta} \delta \epsilon\left(\partial_{j} \pi_{i} \partial^{i} X_{a}^{j}+\partial_{i} \pi_{j} \partial^{i} X_{a}^{j}\right) \\
& -\frac{1}{2}\left(\lambda_{\epsilon} \delta \epsilon^{2}+\lambda_{\pi} \vec{\pi}^{2}\right) \partial^{k} \partial_{k} X_{a}^{t}-\theta_{\epsilon}^{\xi} \epsilon^{i j k} \partial_{j} \pi_{k} \partial_{i} X_{a}^{t}+\frac{i k_{\mathrm{B}} T^{2} \tilde{\lambda}_{\epsilon}}{\partial T / \partial \varepsilon} \delta \epsilon \partial^{i} X_{a}^{t} \partial_{i} X_{a}^{t} \\
& +i k_{\mathrm{B}} T \rho \tilde{\theta}_{\epsilon}^{\eta} \delta \epsilon\left(\partial_{i} X_{a j} \partial^{i} X_{a}^{j}+\partial_{i} X_{a j} \partial^{j} X_{a}^{i}\right)-\frac{2 i T k_{\mathrm{B}} \eta}{\rho}\left(\pi^{i} \partial^{j} X_{a}^{t}+\pi^{j} \partial^{i} X_{a}^{t}\right) \partial_{i} X_{a j} .
\end{aligned}
$$

At the free level, the theory decouples into transverse and longitudinal modes. There is no sound. We still keep the transverse diffusion mode at $D_{\perp}=\eta / \rho$, but the longitudinal diffusion mode now shifts to $D_{\|}=\kappa(\partial T / \partial \varepsilon)$. Since there is no sound, the counting scheme 
slightly changes. Frequency scaling is now controlled by the diffusion modes as $\omega \sim k^{2}$. The fields still scale as $k^{d / 2}$, but the leading derivative interactions terms are only irrelevant by $k^{-d / 2+1}$ and the sub-leading interactions by $k^{-d / 2}$. In $d=2$, this causes the leading interaction couplings (first two terms in $\mathcal{L}_{\text {int }}^{\text {incom }}$ ) to become marginal. In $d=1$, these couplings are actually relevant and the perturbation theory breaks down.

If we were to ignore the momentum modes altogether by setting $\pi^{i}=X_{a}^{i}=0$, we arrive at the theory of energy diffusion derived in [23], truncated to three-point couplings ${ }^{19}$

$$
\begin{aligned}
\mathcal{L}_{\text {free }}^{\text {diff }} & =X_{a}^{t}\left(\partial_{t}-\kappa \frac{\partial T}{\partial \varepsilon} \partial^{i} \partial_{i}\right) \delta \epsilon+i k_{\mathrm{B}} T^{2} \kappa \partial^{i} X_{a}^{t} \partial_{i} X_{a}^{t}, \\
\mathcal{L}_{\text {int }}^{\text {diff }} & =-\frac{1}{2} \lambda_{\epsilon} \delta \epsilon^{2} \partial^{k} \partial_{k} X_{a}^{t}+\frac{i k_{\mathrm{B}} T^{2} \tilde{\lambda}_{\epsilon}}{\partial T / \partial \varepsilon} \delta \epsilon \partial^{i} X_{a}^{t} \partial_{i} X_{a}^{t}
\end{aligned}
$$

This theory has also been studied in detail in [4]. We are only left with the longitudinal diffusion mode at $D_{\|}=\kappa(\partial T / \partial \varepsilon)$. This simplified theory also gets rid of the leadingderivative order interaction terms that were problematic in lower number of dimensions. However, it does serve as a toy model to probe the structure of stochastic field theories.

\section{Coupling to Newton-Cartan sources}

In the previous section, we presented an EFT for $(d+1)$-dimensional Galilean hydrodynamics in terms of $(d+2)$-dimensional uncharged relativistic null fluids. From an implementation viewpoint, this language is immensely helpful as our understanding of relativistic symmetries is much more mature than the Galilean ones. However, it does make the underlying $(d+1)$-dimensional physics more obscure. To remedy this situation, we now present a translation of our results to a manifestly $(d+1)$-dimensional language by "gauge-fixing" the auxiliary null direction. We first introduce the NC backgrounds in section 5.1, seen as a reduction of null backgrounds, along with their coupling to the constitutive relations of classical Galilean hydrodynamics, mirroring section 2. Later in section 5.2, we translate the EFT framework for Galilean hydrodynamics to the NC language following our discussion from section 3. At the end of the day, once the effective action describing our system of interest has been obtained, the path one takes to arrive at the same is immaterial.

\subsection{Classical hydrodynamics with Newton-Cartan sources}

Let us rewind back to section 2.2, where we exploited the embedding of $(d+1)$-dimensional Galilean transformations into $(d+2)$-dimensional Poincare algebra to introduce a set of background sources $g_{\mathrm{MN}}$ coupled to the Galilean conserved currents. To this end, we introduced an auxiliary null direction $V^{\mathrm{M}}$ on the background. To recast these results into a manifestly $(d+1)$-dimensional language, we just need to "undo" this embedding by choosing a coordinate system $\left(x^{\mathrm{M}}\right)=\left(x^{-}, x^{\mu}\right)$ and partially fix the $(d+2)$-dimensional background diffeomorphisms to set $V^{\mathrm{M}}=\delta_{-}^{\mathrm{M}}[46]$. This procedure is formally known as null reduction and yields the so-called Newton-Cartan (NC) formulation of Galilean field theories. The

\footnotetext{
${ }^{19}$ Our $X_{a}^{t}$ field is related to the $\varphi_{a}$ field used in [23] as $X_{a}^{t}=-\varphi_{a}$.
} 
price we pay is that the background now contains a non-invertible "spatial" metric, a torsional spacetime connection, and the Galilean boost symmetry is no longer manifest, making it slightly more technical to implement on its own. In the following, we shall derive the NC results via null reduction. An independent review of the coupling of Galilean field theories to NC sources can be found in [30].

\subsubsection{Newton-Cartan structure}

With the choice of coordinate decomposition mentioned above, a generic null background metric $g_{\mathrm{MN}}$, satisfying $V^{\mathrm{M}} V^{\mathrm{N}} g_{\mathrm{MN}}=g_{--}=0$ and $£_{V} g_{\mathrm{MN}}=\partial_{-} g_{\mathrm{MN}}=0$, can be decomposed as ${ }^{20}$

$$
g_{\text {MN }}(x) \mathrm{d} x^{\mathrm{M}} \mathrm{d} x^{\mathrm{N}}=-2\left(\mathrm{~d} x^{-}-A_{\nu}(x) \mathrm{d} x^{\nu}\right) n_{\mu}(x) \mathrm{d} x^{\mu}+h_{\mu \nu}(x) \mathrm{d} x^{\mu} \mathrm{d} x^{\nu},
$$

In this section, the arguments " $(x)$ " denote the dependence only on the coordinates $x^{\mu}$ and not on $x^{-}$. Here $n_{\mu}$ is called the clock-form or the temporal metric, $h_{\mu \nu}$ the spatial metric, and $A_{\mu}$ is the mass gauge field. They make up the complete set of Newton-Cartan sources. One can check that, in total, these have $(d+1)$ more components than $g_{\mathrm{MN}}$. To fix this redundancy, we require that the spatial metric is degenerate, i.e. given a "frame-velocity vector" $v^{\mu}$ normalised as $v^{\mu} n_{\mu}=1$, we have $v^{\mu} h_{\mu \nu}=0$. This still leaves us with arbitrary redefinitions of $v^{\mu}$ that act as

$$
v^{\mu} \rightarrow v^{\mu}+\psi^{\mu}, \quad h_{\mu \nu} \rightarrow h_{\mu \nu}-2 n_{(\mu} \psi_{\nu)}+n_{\mu} n_{\nu} \psi^{2}, \quad A_{\mu} \rightarrow A_{\mu}+\psi_{\mu}-\frac{1}{2} n_{\mu} \psi^{2},
$$

where $\psi^{\mu}$ is an arbitrary vector satisfying $\psi^{\mu} n_{\mu}=0$; we have denoted $\psi_{\mu}=h_{\mu \nu} \psi^{\nu}$ and $\psi^{2}=\psi^{\mu} \psi^{\nu} h_{\mu \nu}$. This is known as the Milne-boost symmetry. Much of the complication in the NC construction stems from trying to make this symmetry manifest in EFTs. Luckily for us, we get this for free through the null background construction. We can similarly decompose the inverse null background metric as

$$
g^{\mathrm{MN}}(x) \partial_{\mathrm{M}} \otimes \partial_{\mathrm{N}}=-2 v^{\mu}(x)\left(\partial_{\mu}+A_{\mu}(x) \partial_{-}\right) \otimes \partial_{-}+h^{\mu \nu}(x)\left(\partial_{\mu}+A_{\mu}(x) \partial_{-}\right) \otimes\left(\partial_{\nu}+A_{\nu}(x) \partial_{-}\right) .
$$

We have introduced the "inverse spatial metric" $h^{\mu \nu}$, defined via $h^{\mu \nu} h_{\nu \rho}+v^{\mu} n_{\rho}=\delta_{\rho}^{\mu}$ and $n_{\mu} h^{\mu \nu}=0$, which is invariant under Milne boosts. Note that $h^{\mu \nu}$ is not the inverse of $h_{\mu \nu}$. We will denote $h^{\mu}{ }_{\nu}=h^{\mu \lambda} h_{\lambda \nu}$. The NC volume element is defined as $\epsilon^{\mu \nu \rho \sigma}=-\epsilon^{-\mu \nu \rho \sigma}$, so that $\epsilon^{t 123}=1 / \sqrt{\gamma}$ with $\gamma=\operatorname{det}\left(h_{\mu \nu}+n_{\mu} n_{\nu}\right)$.

The residual transformations from the $(d+2)$-dimensional null background diffeomorphisms (2.20) are merely the $(d+1)$-dimensional diffeomorphisms and $\mathrm{U}(1)$ mass gauge transformations

$$
x^{\mu} \rightarrow x^{\prime \mu}(x), \quad x^{-} \rightarrow x^{-}-\Lambda(x) .
$$

These act on the background fields as expected

$$
\begin{array}{ll}
n_{\mu}(x) \rightarrow n_{\mu}^{\prime}\left(x^{\prime}\right)=\frac{\partial x^{\nu}}{\partial x^{\prime \mu}} n_{\nu}(x), & h_{\mu \nu}(x) \rightarrow h_{\mu \nu}^{\prime}\left(x^{\prime}\right)=\frac{\partial x^{\rho}}{\partial x^{\prime \mu}} \frac{\partial x^{\sigma}}{\partial x^{\prime \nu}} h_{\rho \sigma}(x), \\
A_{\mu}(x) \rightarrow A_{\mu}^{\prime}\left(x^{\prime}\right)=\frac{\partial x^{\nu}}{\partial x^{\prime \mu}}\left(A_{\nu}(x)+\partial_{\nu} \Lambda(x)\right), &
\end{array}
$$

\footnotetext{
${ }^{20}$ We use the notations of [30]. In the work of [36-38], the clock-form is equivalently represented by $\tau_{\mu}=-n_{\mu}$. In the work of $[46,54-57]$, the mass gauge field has been represented by $b_{\mu}=A_{\mu}$.
} 
along with

$$
v^{\mu}(x) \rightarrow v^{\prime \mu}\left(x^{\prime}\right)=\frac{\partial x^{\prime \mu}}{\partial x^{\nu}} v^{\nu}(x), \quad h^{\mu \nu}(x) \rightarrow h^{\prime \mu \nu}\left(x^{\prime}\right)=\frac{\partial x^{\prime \mu}}{\partial x^{\rho}} \frac{\partial x^{\prime \nu}}{\partial x^{\sigma}} h^{\rho \sigma}(x) .
$$

These transformation properties follow directly from eq. (2.21).

The NC connection $\tilde{\Gamma}_{\mu \nu}^{\lambda}$ is defined such that the associated covariant derivative operator $\tilde{\nabla}_{\mu}$ satisfies $\tilde{\nabla}_{\mu} n_{\nu}=\tilde{\nabla}_{\mu} h^{\nu \rho}=0$. It is not possible to construct such a connection that is simultaneously invariant under mass gauge transformations and Milne boosts as well. Sacrificing the Milne boosts, we can write down one such connection ${ }^{21}$

$$
\tilde{\Gamma}_{\mu \nu}^{\lambda}=v^{\lambda} \partial_{\mu} n_{\nu}+\frac{1}{2} h^{\lambda \rho}\left(\partial_{\mu} h_{\nu \rho}+\partial_{\nu} h_{\mu \rho}-\partial_{\rho} h_{\mu \nu}\right)+n_{(\mu} F_{\nu) \rho} h^{\rho \lambda} .
$$

Here $F_{\mu \nu}=2 \partial_{[\mu} A_{\nu]}$ is the mass gauge field strength. Notice that the NC connection is torsional: $2 \tilde{\Gamma}_{[\mu \nu]}^{\lambda}=v^{\lambda} F_{\mu \nu}^{n}$, where $F_{\mu \nu}^{n}=2 \partial_{[\mu} n_{\nu]}$ is the temporal torsion. We can also generically introduce spatial and "mass" torsion, but it is not imposed upon us by the framework (see [57]). It is easy to see that the connection is not left invariant by Milne boosts (5.2)

$$
\tilde{\Gamma}_{\mu \nu}^{\lambda} \rightarrow \tilde{\Gamma}_{\mu \nu}^{\lambda}+\frac{1}{2} \psi^{\lambda} F_{\mu \nu}^{n}-\psi_{(\mu} F_{\nu) \rho}^{n} h^{\rho \lambda}+\frac{1}{2} \psi^{2} n_{(\mu} F_{\nu) \rho}^{n} h^{\rho \lambda} .
$$

The null background Levi-Civita connection $\Gamma_{\mathrm{MN}}^{\mathrm{R}}$ can be decomposed in terms of $\tilde{\Gamma}_{\mu \nu}^{\lambda}$ as

$$
\begin{aligned}
& \Gamma_{\mathrm{MN}}^{-} \mathrm{d} x^{\mathrm{M}} \mathrm{d} x^{\mathrm{N}}=\left(h_{\lambda(\nu} \tilde{\nabla}_{\mu)} v^{\lambda}-\tilde{\nabla}_{(\mu} A_{\nu)}\right) \mathrm{d} x^{\mu} \mathrm{d} x^{\nu}-\left(\mathrm{d} x^{-}-A_{\nu} \mathrm{d} x^{\nu}\right)\left(v^{\rho}-h^{\rho \lambda} A_{\lambda}\right) F_{\rho \mu}^{n} \mathrm{~d} x^{\mu}, \\
& \Gamma_{\mathrm{MN}}^{\lambda} \mathrm{d} x^{\mathrm{M}} \mathrm{d} x^{\mathrm{N}}=\tilde{\Gamma}_{(\mu \nu)}^{\lambda} \mathrm{d} x^{\mu} \mathrm{d} x^{\nu}+\left(\mathrm{d} x^{-}-A_{\nu} \mathrm{d} x^{\nu}\right) h^{\lambda \rho} F_{\rho \mu}^{n} \mathrm{~d} x^{\mu} .
\end{aligned}
$$

Note that $h_{\nu \lambda} \tilde{\nabla}_{\mu} v^{\lambda}=\frac{1}{2} F_{\mu \nu}-v^{\rho}\left(n_{(\mu} F_{\nu) \rho}+\partial_{(\mu} h_{\nu) \rho}-\frac{1}{2} \partial_{\rho} h_{\mu \nu}\right)$ and $\tilde{\nabla}_{\lambda} h_{\mu \nu}=-2 n_{(\mu} h_{\nu) \rho} \tilde{\nabla}_{\lambda} v^{\rho}$. Also that $\Gamma_{\mathrm{M} \nu}^{\mathrm{M}}=\tilde{\Gamma}_{\mu \nu}^{\mu}+F_{\nu \mu}^{n} v^{\mu}=\frac{1}{\sqrt{\gamma}} \partial_{\nu} \sqrt{\gamma}$. These identities are helpful in the explicit derivations below.

To revert back to a flat background, we just need to set $n_{\mu}=\delta_{\mu}^{t}, v^{\mu}=\delta_{t}^{\mu}, A_{\mu}=0, h_{\mu \nu}=$ $\delta_{\mu i} \delta_{\nu}^{i}$, and $h^{\mu \nu}=\delta^{\mu i} \delta_{i}^{\nu}$. This also sets the connection $\tilde{\Gamma}_{\mu \nu}^{\lambda}=0$, along with $F_{\mu \nu}=F_{\mu \nu}^{n}=0$.

\subsubsection{Coupling to Galilean field theories}

We can reduce the null conservation equations (2.18) to obtain the covariant version of the Galilean conservation equations in the NC language. To this end, we introduce the covariant mass current $\rho^{\mu}$, energy current $\epsilon^{\mu}$, and spatial stress tensor $\tau^{\mu \nu}$ in terms of the higher dimensional energy-momentum tensor $T^{\mathrm{MN}}$ as

$$
\rho^{\mu}=T^{\mu \nu} n_{\nu}, \quad \epsilon^{\mu}=T^{\mu-}-T^{\mu \rho} A_{\rho}, \quad \tau^{\mu \nu}=h_{\rho}^{\mu} h_{\sigma}^{\nu} T^{\rho \sigma} .
$$

Note that $\tau^{\mu \nu}=\tau^{\nu \mu}$ and $\tau^{\mu \nu} n_{\nu}=0$. It is also convenient to define the momentum density $\pi^{\mu}=h_{\nu}^{\mu} \rho^{\nu}$ that is equal to the mass flux. These definitions are chosen so that they are invariant under mass gauge transformations, however, $\epsilon^{\mu}$ and $\tau^{\mu \nu}$ are consequently

\footnotetext{
${ }^{21}$ It is known that this connection is not unique; see [30] for a detailed discussion.
} 
not boost-invariant. This is what one would physically expect, because the notion of energy and momentum depends on the choice of the Galilean observer. The conservation equations (2.18) then become

$$
\begin{array}{cl}
\text { Energy conservation: } & \left(\tilde{\nabla}_{\mu}+F_{\mu \lambda}^{n} v^{\lambda}\right) \epsilon^{\mu}=v^{\nu} F_{\nu \mu}^{n} \epsilon^{\mu}-\left(v^{\mu} \pi^{\lambda}+\tau^{\mu \lambda}\right) h_{\lambda \nu} \tilde{\nabla}_{\mu} v^{\nu}, \\
\text { Momentum conservation: } & \left(\tilde{\nabla}_{\mu}+F_{\mu \lambda}^{n} v^{\lambda}\right)\left(v^{\mu} \pi^{\nu}+\tau^{\mu \nu}\right)=-h^{\nu \lambda} F_{\lambda \mu}^{n} \epsilon^{\mu}-\rho^{\mu} \tilde{\nabla}_{\mu} v^{\nu}, \\
\text { Continuity equation: } \quad & \left(\tilde{\nabla}_{\mu}+F_{\mu \lambda}^{n} v^{\lambda}\right) \rho^{\mu}=0 .
\end{array}
$$

These are the covariant version of the Galilean conservation equations (2.2). Aside from the torsional contributions coupled to $F_{\mu \nu}^{n}$, the energy and momentum conservation is sourced by the "pseudo-power" and "pseudo-force" contributions coupled to the derivatives of frame velocity.

Given some action $S$ describing a Galilean field theory, the coupling between various currents and sources takes the form following from eq. $(2.23)^{22}$

$$
\delta S=\int \mathrm{d}^{d+1} x \sqrt{\gamma}\left(\rho^{\mu} \delta A_{\mu}-\epsilon^{\mu} \delta n_{\mu}+\left(v^{\mu} \pi^{\nu}+\frac{1}{2} \tau^{\mu \nu}\right) \delta h_{\mu \nu}\right) .
$$

Conservation equations (5.9) follow from requiring the action to be invariant under the Galilean transformations (5.4). On the other hand, invariance under Milne boosts (5.2) leads to the identity between momentum density and mass flux $\pi^{\mu}=h^{\mu}{ }_{\nu} \rho^{\nu}$. The retarded two-point functions of various quantities follow from the reduction of eq. (2.24). Up to contact terms, we find

$$
\begin{aligned}
G_{\rho^{\mu} \rho^{\nu}}^{\mathrm{R}} & =\frac{1}{\sqrt{\gamma}} \frac{\delta\left(\sqrt{\gamma} \rho^{\mu}\right)}{\delta A_{\nu}}, & G_{\epsilon^{\mu} \epsilon^{\nu}}^{\mathrm{R}} & =-\frac{1}{\sqrt{\gamma}} \frac{\delta\left(\sqrt{\gamma} \epsilon^{\mu}\right)}{\delta n_{\nu}}, \\
G_{\tau^{\mu \nu} \tau^{\rho \sigma}}^{\mathrm{R}} & =h^{\mu}{ }_{\lambda} h^{\nu}{ }_{\tau} h^{\rho}{ }_{\alpha} h^{\sigma}{ }_{\beta} \frac{2}{\sqrt{\gamma}} \frac{\delta\left(\sqrt{\gamma} \tau^{\lambda \tau}\right)}{\delta h_{\alpha \beta}}, & & \\
G_{\rho^{\mu} \epsilon^{\nu}}^{\mathrm{R}} & =-\frac{1}{\sqrt{\gamma}} \frac{\delta\left(\sqrt{\gamma} \rho^{\mu}\right)}{\delta n_{\nu}}, & G_{\epsilon^{\mu} \rho^{\nu}}^{\mathrm{R}} & =\frac{1}{\sqrt{\gamma}} \frac{\delta\left(\sqrt{\gamma} \epsilon^{\mu}\right)}{\delta A_{\nu}}, \\
G_{\tau^{\mu \nu} \rho^{\lambda}}^{\mathrm{R}} & =h^{\mu}{ }_{\rho} h^{\nu}{ }_{\sigma} \frac{2}{\sqrt{\gamma}} \frac{\delta\left(\sqrt{\gamma} \rho^{\lambda}\right)}{\delta h_{\rho \sigma}}, & G_{\rho^{\lambda} \tau^{\mu \nu}}^{\mathrm{R}} & =h^{\mu}{ }_{\rho} h^{\nu}{ }_{\sigma} \frac{1}{\sqrt{\gamma}} \frac{\delta\left(\sqrt{\gamma} \tau^{\rho \sigma}\right)}{\delta A_{\lambda}} \\
G_{\tau^{\mu \nu} \epsilon^{\lambda}}^{\mathrm{R}} & =h^{\mu}{ }_{\rho} h^{\nu}{ }_{\sigma} \frac{2}{\sqrt{\gamma}} \frac{\delta\left(\sqrt{\gamma} \epsilon^{\lambda}\right)}{\delta h_{\rho \sigma}}, & G_{\epsilon^{\lambda} \tau^{\mu \nu}}^{\mathrm{R}} & =-h^{\mu}{ }_{\rho} h^{\nu}{ }_{\sigma} \frac{1}{\sqrt{\gamma}} \frac{\delta\left(\sqrt{\gamma} \tau^{\rho \sigma}\right)}{\delta n_{\lambda}} .
\end{aligned}
$$

The factors of $h^{\mu}{ }_{\nu}$ project out the appropriate spatial components for the spatial stress tensor $\tau^{\mu \nu}$ on the left hand side.

Let us say that we are provided with a "lab frame" characterised by the time coordinate $t$. We can use this to fix the Milne boost symmetry (5.2) exactly by choosing the Galilean

\footnotetext{
${ }^{22}$ These can also be stated in the form advocated in [30]. Note that $v^{\mu} \delta h_{\mu \nu}=-\delta h_{\mu \nu} \delta v^{\mu}$ and $\delta h_{\mu \nu}=$ $-h_{\mu \rho} h_{\nu \sigma} \delta h^{\rho \sigma}$, which recasts the integrand into $\rho^{\mu} \delta A_{\mu}-\epsilon^{\mu} \delta n_{\mu}-\pi_{\mu} \delta v^{\mu}-\frac{1}{2} \tau_{\mu \nu} \delta h^{\mu \nu}$, where $\pi_{\mu}=h_{\mu \nu} \pi^{\nu}$ and $\tau_{\mu \nu}=h_{\mu \rho} h_{\nu \sigma} \tau^{\rho \sigma}$.
} 
frame velocity to be $v^{\mu} \propto \delta_{t}^{\mu}$. The background sources then become

$$
\begin{array}{rlrl}
A_{\mu} \mathrm{d} x^{\mu} & =A_{t} \mathrm{~d} t+A_{i} \mathrm{~d} x^{i}, & n_{\mu} \mathrm{d} x^{\mu} & =n_{t} \mathrm{~d} t+n_{i} \mathrm{~d} x^{i}, \\
v^{\mu} \partial_{\mu} & =\frac{1}{n_{t}} \partial_{t}, & h_{\mu \nu} \mathrm{d} x^{\mu} \mathrm{d} x^{\nu}=h_{i j} \mathrm{~d} x^{i} \mathrm{~d} x^{j}, \\
h^{\mu \nu} \partial_{\mu} \otimes \partial_{\nu} & =\frac{h^{i j} n_{i} n_{j}}{n_{t}^{2}} \partial_{t} \otimes \partial_{t}-2 \frac{h^{i j} n_{j}}{n_{t}} \partial_{t} \otimes \partial_{i}+h^{i j} \partial_{i} \otimes \partial_{j}, \\
h_{\nu}^{\mu} \mathrm{d} x^{\nu} \partial_{\mu} & =-\frac{n_{i}}{n_{t}} \mathrm{~d} x^{i} \partial_{t}+\delta_{i}^{j} \mathrm{~d} x^{i} \partial_{j}, & \gamma & =n_{t}^{2} h .
\end{array}
$$

Here $h^{i j}$ is the inverse of $h_{i j}$ and $h=\operatorname{det} h_{i j}$. The action variation (5.10) takes a more natural form

$$
\delta S=\int \mathrm{d}^{d+1} x n_{t} \sqrt{h}\left(\rho^{t} \delta A_{t}+\rho^{i} \delta A_{i}-\epsilon^{t} \delta n_{t}-\epsilon^{i} \delta n_{i}+\frac{1}{2} \tau^{i j} \delta h_{i j}\right)
$$

with $h_{i j}$ sourcing the stress tensor. The response functions follow accordingly from eq. (5.11).

\subsubsection{Hydrodynamics on curved spacetime}

We can use the generic discussion above to reduce the constitutive relations of Galilean hydrodynamics to the NC language. We decompose the null fluid velocity as $u^{\mathrm{M}} \partial_{\mathrm{M}}=u^{-} \partial_{-}+$ $u^{\mu} \partial_{\mu}$. The normalisation conditions (2.27) imply that $u^{\mu} n_{\mu}=1$ and $u^{-}=\frac{1}{2} \vec{u}^{2}+u^{\mu} A_{\mu}$, where $\vec{u}^{2}=u^{\mu} u^{\nu} h_{\mu \nu}$. Here $u^{\mu}$ is understood as the covariant velocity of the Galilean fluid. We can further define the spatial part of the fluid velocity as $\vec{u}^{\mu}=h_{\nu}^{\mu} u^{\nu}=u^{\mu}-v^{\mu}$ with $\vec{u}_{\mu}=h_{\mu \nu} u^{\nu}$. The null fluid constitutive relations and entropy current from (2.25) can be reduced to obtain the respective $\mathrm{NC}$ versions ${ }^{23}$

$$
\begin{aligned}
\rho^{\mu} & =\rho u^{\mu}, \quad \epsilon^{\mu}=\left(\varepsilon+\frac{1}{2} \rho \vec{u}^{2}\right) u^{\mu}+q^{\mu}+t^{\mu \nu} \vec{u}_{\nu}, \quad \tau^{\mu \nu}=\left(\rho \vec{u}^{\mu} \vec{u}^{\nu}+t^{\mu \nu}\right), \\
s^{\mu} & =s u^{\mu}+\frac{1}{T} q^{\mu}+\Upsilon^{\mu} .
\end{aligned}
$$

The comoving heat flux $q^{\mu}$ and stress tensor $t^{\mu \nu}$ satisfy $q^{\mu} n_{\mu}=t^{\mu \nu} n_{\nu}=0$ and $t^{\mu \nu}=t^{\nu \mu}$, while the non-canonical entropy current $\Upsilon^{\mu}$ is un-normalised. Up to one-derivative order,

\footnotetext{
${ }^{23}$ Here $\epsilon^{\mu}$ and $\tau^{\mu \nu}$ are defined in an arbitrary Galilean frame of reference characterised by $v^{\mu}$. In hydrodynamics, we can explicitly fix the Milne boost symmetry by choosing $v^{\mu}=u^{\mu}$ or $\vec{u}^{\mu}=0$. This is equivalent to working in the local rest frame of the fluid. We can always return to an arbitrary frame by performing an inverse Milne boost with $\psi^{\mu}=-\vec{u}^{\mu}$. However, with this choice, the flat background limit is slightly non-trivial

$$
\begin{aligned}
& \left.n_{\mu} \mathrm{d} x^{\mu}\right|_{\text {flat }}=\mathrm{d} t,\left.\quad h_{\mu \nu} \mathrm{d} x^{\mu} \mathrm{d} x^{\nu}\right|_{\text {flat }}=\vec{u}^{2} \mathrm{~d} t^{2}-2 u_{i} \mathrm{~d} x^{i} \mathrm{~d} t+\delta_{i j} \mathrm{~d} x^{i} \mathrm{~d} x^{j}, \quad h^{\mu \nu} \partial_{\mu} \otimes \partial_{\nu}=\delta^{i j} \partial_{i} \otimes \partial_{j}, \\
& \left.A_{\mu} \mathrm{d} x^{\mu}\right|_{\text {flat }}=-\frac{1}{2} \vec{u}^{2} \mathrm{~d} t+u_{i} \mathrm{~d} x^{i},\left.\quad \tilde{\Gamma}_{\mu \nu}^{\lambda}\right|_{\text {flat }}=0 .
\end{aligned}
$$

Since the energy and momentum defined in fluid's rest frame are not individually conserved, we have chosen to instead work in an arbitrary lab frame of reference in the main text. 
these are given by the reduction of eq. (2.29) leading to

$$
\begin{aligned}
t^{\mu \nu}= & p h^{\mu \nu}-\eta \sigma^{\mu \nu}-\zeta h^{\mu \nu}\left(\tilde{\nabla}_{\lambda}+F_{\lambda \rho}^{n} v^{\rho}\right) u^{\lambda}, \\
q^{\mu}= & -\kappa h^{\mu \nu}\left(\partial_{\nu} T+T F_{\nu \rho}^{n} u^{\rho}\right)-\frac{1}{2} \lambda_{\Omega} \epsilon^{\mu \nu \rho \sigma} n_{\nu} \Omega_{\rho \sigma}+\frac{1}{2} \lambda_{H} \epsilon^{\mu \nu \rho \sigma} n_{\nu} F_{\rho \sigma}^{n}, \\
\Upsilon^{\mu}= & -\frac{a_{2}}{2 T} \epsilon^{\mu \nu \rho \sigma} n_{\nu} \Omega_{\rho \sigma}+\frac{a_{1}}{4 T} \epsilon^{\mu \nu \rho \sigma} n_{\nu} F_{\rho \sigma}^{n} \\
& +\frac{a_{0}}{2 T} \epsilon^{\mu \nu \rho \sigma}\left(\vec{u}_{\nu}+A_{\nu}-\frac{1}{2} \vec{u}^{2} n_{\nu}\right) \partial_{\rho}\left(\vec{u}_{\sigma}+A_{\sigma}-\frac{1}{2} \vec{u}^{2} n_{\sigma}\right),
\end{aligned}
$$

where the fluid shear and vorticity tensors are given as

$$
\begin{aligned}
& \sigma^{\mu \nu}=2\left(h^{\rho(\mu} h_{\lambda}^{\nu)}-\frac{1}{d} h^{\mu \nu} h_{\lambda}^{\rho}\right)\left(\tilde{\nabla}_{\rho} u^{\lambda}+u^{\lambda} F_{\rho \sigma}^{n} u^{\sigma}\right), \\
& \Omega_{\mu \nu}=h_{\mu}^{\rho} h_{\nu}^{\sigma}\left(2 \partial_{[\rho} \vec{u}_{\sigma]}+F_{\rho \sigma}-\frac{1}{2} \vec{u}^{2} F_{\rho \sigma}^{n}\right) .
\end{aligned}
$$

All the coefficients appearing here are functions of $T$ and $\mu$. The thermodynamic coefficients $p, \varepsilon, \rho$, and $s$ satisfy the relations (2.12), the dissipative transport coefficients $\eta, \zeta$, and $\kappa$ are required to be positive semi-definite, while the adiabatic transport coefficients $\lambda_{\Omega}$, $\lambda_{H}, a_{0}, a_{1}$, and $a_{2}$ are given in terms of three arbitrary constants $K_{0}, K_{1}, K_{2}$ according to eq. (2.30). The hydrodynamic equations of motion in the Newton-Cartan language are given by substituting the constitutive relations into eq. (5.9). These constitutive relations satisfy the second law of thermodynamics

$$
\left(\tilde{\nabla}_{\mu}+n_{\mu \lambda} v^{\lambda}\right) s^{\mu} \geq 0
$$

on the solutions of the equations of motion.

As discussed in section 2.3, the constitutive relations specified above are written in the "mass frame". One can arbitrarily depart from this choice of frame, however, by redefining $u^{\mu}, T$, and $\mu$ according to eq. (2.31). For instance, the thermodynamic mass frame is arrived at by keeping the definitions of $T$ and $\mu$ intact, but redefining the fluid velocity according to

$$
u^{\mu} \rightarrow u^{\mu}-\frac{a_{0}}{\rho} \epsilon^{\mu \nu \rho \sigma} n_{\nu} \Omega_{\rho \sigma}+\frac{a_{2}}{2 \rho} \epsilon^{\mu \nu \rho \sigma} n_{\nu} F_{\rho \sigma}^{n} .
$$

The transformed set of constitutive relations are given by the reduction of eqs. (2.32) and (2.36)

$$
\begin{aligned}
\rho^{\mu} & =\rho u^{\mu}+j^{\mu}, & \epsilon^{\mu} & =\left(\varepsilon+\frac{1}{2} \rho \vec{u}^{2}+j^{\nu} \vec{u}_{\nu}\right) u^{\mu}+q^{\mu}+t^{\mu \nu} \vec{u}_{\nu}+\frac{1}{2} \vec{u}^{2} j^{\mu}, \\
\tau^{\mu \nu} & =\rho \vec{u}^{\mu} \vec{u}^{\nu}+t^{\mu \nu}+2 \vec{u}^{(\mu} j^{\nu)}, & s^{\mu} & =s u^{\mu}-\frac{\mu}{T} j^{\mu}+\frac{1}{T} q^{\mu}+\Upsilon^{\mu},
\end{aligned}
$$


where

$$
\begin{aligned}
t^{\mu \nu}= & p h^{\mu \nu}-\eta \sigma^{\mu \nu}-\zeta h^{\mu \nu}\left(\tilde{\nabla}_{\lambda}+F_{\lambda \rho}^{n} v^{\rho}\right) u^{\lambda}, \\
j^{\mu}= & -\frac{a_{0}}{2} \epsilon^{\mu \nu \rho \sigma} n_{\nu} \Omega_{\rho \sigma}+\frac{a_{2}}{2} \epsilon^{\mu \nu \rho \sigma} n_{\nu} F_{\rho \sigma}^{n}, \\
q^{\mu}= & -\kappa h^{\mu \nu}\left(\partial_{\nu} T+T F_{\nu \rho}^{n} u^{\rho}\right)-\frac{a_{2}}{2} \epsilon^{\mu \nu \rho \sigma} n_{\nu} \Omega_{\rho \sigma}+\frac{a_{1}}{2} \epsilon^{\mu \nu \rho \sigma} n_{\nu} F_{\rho \sigma}^{n}, \\
\Upsilon^{\mu}= & -\frac{a_{2}}{2 T} \epsilon^{\mu \nu \rho \sigma} n_{\nu} \Omega_{\rho \sigma}+\frac{a_{1}}{4 T} \epsilon^{\mu \nu \rho \sigma} n_{\nu} F_{\rho \sigma}^{n} \\
& +\frac{a_{0}}{2 T} \epsilon^{\mu \nu \rho \sigma}\left(\vec{u}_{\nu}+A_{\nu}-\frac{1}{2} \vec{u}^{2} n_{\nu}\right) \partial_{\rho}\left(\vec{u}_{\sigma}+A_{\sigma}-\frac{1}{2} \vec{u}^{2} n_{\sigma}\right) .
\end{aligned}
$$

In this frame, the constitutive relations can be checked to satisfy the adiabaticity equation obtained by reducing eq. (2.41)

$$
\left(\tilde{\nabla}_{\mu}+n_{\mu \lambda} v^{\lambda}\right) N^{\mu}=\rho^{\mu} \delta_{\mathscr{B}} A_{\mu}-\epsilon^{\mu} \delta_{\mathscr{B}} n_{\mu}+\left(v^{\mu} p^{\nu}+\frac{1}{2} \tau^{\mu \nu}\right) \delta_{\mathscr{B}} h_{\mu \nu}+\Delta, \quad \Delta \geq 0,
$$

with the free energy current

$$
k_{\mathrm{B}} T N^{\mu}=T s^{\mu}+\rho^{\mu}\left(\mu-\frac{1}{2} \vec{u}^{2}\right)-\epsilon^{\mu}+\left(v^{\mu} \pi^{\nu}+\tau^{\mu \nu}\right) \vec{u}_{\nu}=p u^{\mu}+T \Upsilon^{\mu} .
$$

The operator $\delta_{\mathscr{B}}$ above combines a Lie derivative along $\beta^{\mu}=u^{\mu} /\left(k_{\mathrm{B}} T\right)$, denoted by $£_{\beta}$, and a gauge shift along $\Lambda_{\beta}=-\beta^{-}=\left(\mu-\frac{1}{2} u^{2}-A_{\mu} u^{\mu}\right) /\left(k_{\mathrm{B}} T\right)$. Explicitly

$$
\begin{aligned}
\delta_{\mathscr{B}} n_{\mu} & =£_{\beta} n_{\mu}=-\left(\frac{1}{k_{\mathrm{B}} T^{2}} \partial_{\mu} T+F_{\mu \nu}^{n} \beta^{\nu}\right), \\
\delta_{\mathscr{B}} h_{\mu \nu} & =£_{\beta} h_{\mu \nu}=2\left(h_{\lambda(\mu} \tilde{\nabla}_{\nu)} \beta^{\lambda}-n_{(\mu} h_{\nu) \rho} \beta^{\lambda} \tilde{\nabla}_{\lambda} v^{\rho}\right), \\
\delta_{\mathscr{B}} A_{\mu} & =£_{\beta} A_{\mu}+\partial_{\mu} \Lambda_{\beta}=\partial_{\mu}\left(\frac{\mu-\frac{1}{2} \vec{u}^{2}}{k_{\mathrm{B}} T}\right)-F_{\mu \nu} \beta^{\nu} .
\end{aligned}
$$

The discussion of discrete symmetries is precisely the same as section 2.4. Requiring the underlying microscopic theory to be invariant under $\Theta=$ T symmetry does not lead to any new constraints on the constitutive relations, while $\Theta=$ PT switches off the entire parity-violating sector. See table 1 for the action of discrete symmetries on various fields. These constraints follow from requiring the associated equilibrium partition function to respect $\Theta$ [54]. Note that the constitutive relations themselves will not, in general, respect any kind of time reversal symmetry due to dissipation. The effective field theory framework deals with these discrete symmetries more carefully.

\subsection{Schwinger-Keldysh effective field theory}

We can extend the null reduction philosophy above to the EFT as well. Compared to our discussion in section 3 , we need to pick a coordinate system $\left(\sigma^{\mathrm{A}}\right)=\left(\sigma^{-}, \sigma^{\alpha}\right)$ on the fluid worldvolume such that $\mathbb{V}^{\mathrm{A}}(\sigma)=\delta_{-}^{\mathrm{A}}$, and identify the reference mass chemical shift field $\Lambda_{\beta}(\sigma)=-\beta^{-}(\sigma)$. Similarly on the SK spacetimes we set $V_{s}^{\mathrm{M}}\left(X_{s}\right)=\delta_{-}^{\mathrm{M}}$ and identify the auxiliary coordinate field with the $\mathrm{U}(1)$ mass phase $\varphi_{s}(s)$ via $X_{s}^{-}(\sigma)=\sigma^{-}-\varphi_{s}(\sigma)$. This follows through to the physical spacetime with the choice of average coordinate basis $\left(x^{\mathrm{M}}\right)=$ $\left(x^{-}, x^{\mu}\right)$ such that $V^{\mu}(x)=\delta_{-}^{\mu}$; we can identify the thermal shift field $\Lambda_{\beta}(x)=-\beta^{-}(x)$. The details will follow. 


\subsubsection{Effective field theory on fluid worldvolume}

We start with a $(d+1)$-dimensional "fluid worldvolume" with coordinates $\sigma^{\alpha}$, interpreted as a set of internal spacetime labels associated with each "fluid element". On this spacetime lives the dynamical fields of the theory: $X_{s}^{\mu}(\sigma)$ and $\varphi_{s}(\sigma)\left(=\sigma^{-}-X_{s}^{-}(\sigma)\right)$ with $s=1,2$, which should be understood as the SK double copies of spacetime coordinates and U(1) mass phases, respectively, of a given fluid element. Decomposing $X_{1,2}^{\mu}=X_{r}^{\mu} \pm \hbar / 2 X_{a}^{\mu}$ and $\varphi_{1,2}=\varphi_{r} \pm \hbar / 2 \varphi_{a}$, the average combinations $X_{r}^{\mu}(\sigma)$ and $\varphi_{r}(\sigma)\left(=\sigma^{-}-X_{r}^{-}(\sigma)\right)$ are understood as the true physical spacetime coordinates and $\mathrm{U}(1)$ phase of the fluid elements, while $X_{a}^{\mu}(\sigma)$ and $\varphi_{a}(\sigma)\left(=-X_{a}^{-}(\sigma)\right)$ as the associated stochastic noise. The worldvolume also features a fixed thermal time vector field $\beta^{\alpha}(\sigma)$ and a mass chemical shift field $\Lambda_{\beta}(\sigma)\left(=-\beta^{-}(\sigma)\right)$, which determine the global rest frame and reference chemical potential associated with the global thermal state.

The EFT for Galilean hydrodynamics must be invariant under global Galilean transformations of the coordinates $X_{s}^{\mu}(\sigma)$ and global U(1) shifts of the phases $\varphi_{s}(\sigma)$, acting independently on the two SK spacetimes. To probe the associated Noether currents, we can introduce double copies of NC sources similar to eq. (5.1): clock forms $n_{s \mu}\left(X_{s}\right)$, spatial metrics $h_{s \mu \nu}\left(X_{s}\right)$, and mass gauge fields $A_{s \mu}\left(X_{s}\right)$. We can also introduce the inverse spatial metrics $h_{s}^{\mu \nu}\left(X_{s}\right)$ and frame velocities $v_{s}^{\mu}\left(X_{s}\right)$ associated with these sources using the normalisation conditions $v_{s}^{\mu} n_{s \mu}=1, h_{s \mu \nu} v_{s}^{\nu}=0, h_{s}^{\mu \nu} n_{s \nu}=0$, and $h_{s \mu \nu} h_{s}^{\nu \rho}+n_{s \mu} v_{s}^{\rho}=\delta_{\mu}^{\rho}$. These sources have a Milne redundancy (5.2) among them, acting independently on the two copies. With the background sources in place, the system is required to be invariant under local SK spacetime diffeomorphisms and gauge transformations (following from eq. (3.1))

$$
X_{s}^{\mu}(\sigma) \rightarrow X_{s}^{\prime \mu}\left(X_{s}(\sigma)\right), \quad \varphi_{s}(\sigma) \rightarrow \varphi_{s}(\sigma)-\Lambda_{s}\left(X_{s}(\sigma)\right)
$$

which act on the background sources as usual according to eq. (5.4). To make the symmetries (5.23) manifest, we can perform a pullback onto the fluid worldvolume, along with a mass gauge transformation for the gauge fields, to obtain

$$
\begin{aligned}
& \mathrm{n}_{s \alpha}(\sigma)=n_{s \mu}\left(X_{s}(\sigma)\right) \partial_{\alpha} X_{s}^{\mu}(\sigma), \\
& \mathbb{A}_{s \alpha}(\sigma)=A_{s \mu}\left(X_{s}(\sigma)\right) \partial_{\alpha} X_{s}^{\mu}(\sigma)+\partial_{\alpha} \varphi_{s}(\sigma),
\end{aligned} \quad \mathfrak{h}_{s \alpha \beta}(\sigma)=h_{s \mu \nu}\left(X_{s}(\sigma)\right) \partial_{\alpha} X_{s}^{\mu}(\sigma) \partial_{\beta} X_{s}^{\nu}(\sigma),
$$

and similarly for the inverse spatial metrics and the frame velocities. However, these quantities still transform under the Milne boosts as

$$
\begin{aligned}
\mathfrak{h}_{s \alpha \beta}(\sigma) & \rightarrow \mathfrak{h}_{s \alpha \beta}(\sigma)-2 \mathfrak{n}_{(\alpha}(\sigma) \psi_{s \beta)}(\sigma)+\mathfrak{n}_{s \alpha}(\sigma) \mathfrak{n}_{s \beta}(\sigma) \psi_{s}^{2}(\sigma), \\
\mathbb{A}_{s \alpha}(\sigma) & \rightarrow \mathbb{A}_{s \alpha}(\sigma)+\psi_{s \alpha}(\sigma)-\frac{1}{2} \mathfrak{n}_{s \alpha}(\sigma) \psi_{s}^{2}(\sigma),
\end{aligned}
$$

where $\psi_{s \alpha}=\mathfrak{h}_{s \alpha \beta} \psi_{s}^{\beta}$ and $\psi_{s}^{2}=\mathfrak{h}_{s \alpha \beta} \psi_{s}^{\alpha} \psi_{s}^{\beta}$ for some vectors $\psi_{s}^{\alpha}$ satisfying $\psi_{s}^{\alpha} \mathrm{n}_{s \alpha}=0$.

The internal labelling scheme for fluid elements, i.e. the coordinates $\sigma^{\alpha}$ on the worldvolume, can be arbitrarily redefined without changing any physics. Similarly, the U(1) phases $\varphi_{s}(\sigma)$ can be arbitrarily shifted among the fluid elements. This leads to a local invariance of the theory under diffeomorphisms and $\mathrm{U}(1)$ gauge transformations on the 
fluid worldvolume (following from eq. (3.4))

$$
\sigma^{\alpha} \rightarrow \sigma^{\prime \alpha}(\sigma), \quad \varphi_{s}(\sigma) \rightarrow \varphi_{s}(\sigma)+\lambda(\sigma)
$$

Note that the two phases are required to shift simultaneously. These transformations act naturally on all the fluid worldvolume objects

$$
\begin{array}{rlrl}
\mathfrak{m}_{s \alpha}(\sigma) \rightarrow \mathfrak{m}_{s \alpha}^{\prime}\left(\sigma^{\prime}\right) & =\frac{\partial \sigma^{\beta}}{\partial \sigma^{\prime \alpha}} \mathfrak{n}_{s \beta}(\sigma), & \mathfrak{h}_{s \alpha \beta}(\sigma) \rightarrow \mathfrak{h}_{s \alpha \beta}^{\prime}\left(\sigma^{\prime}\right)=\frac{\partial \sigma^{\gamma}}{\partial \sigma^{\prime \alpha}} \frac{\partial \sigma^{\delta}}{\partial \sigma^{\prime \beta}} \mathfrak{h}_{s \gamma \delta}(\sigma), \\
\mathbb{A}_{s \alpha}(\sigma) \rightarrow \mathbb{A}_{s \alpha}^{\prime}\left(\sigma^{\prime}\right) & =\frac{\partial \sigma^{\beta}}{\partial \sigma^{\prime \alpha}}\left(\mathbb{A}_{s \beta}(\sigma)+\partial_{\beta} \lambda(\sigma)\right), & & \\
\beta^{\alpha}(\sigma) & \rightarrow \mathbb{\beta}^{\prime \alpha}\left(\sigma^{\prime}\right)=\frac{\partial \sigma^{\prime \alpha}(\sigma)}{\partial \sigma^{\beta}} \mathbb{\beta}^{\beta}(\sigma), & \Lambda_{\beta}(\sigma) \rightarrow \Lambda_{\beta}^{\prime}\left(\sigma^{\prime}\right)=\Lambda_{\beta}(\sigma)-\beta^{\alpha}(\sigma) \partial_{\alpha} \lambda(\sigma),
\end{array}
$$

which can be implemented using the usual techniques. Note that the difference combination of the pullback of mass gauge fields $\mathbb{A}_{a \alpha}=\left(\mathbb{A}_{1 \alpha}-\mathbb{A}_{2 \alpha}\right) / \hbar$ is gauge invariant, while the dependence on the average combination $\mathbb{A}_{r \alpha}=\left(\mathbb{A}_{1 \alpha}+\mathbb{A}_{2 \alpha}\right) / 2$ must only come via the "time-component" $\mathbb{\beta}^{\alpha} \mathbb{A}_{r \alpha}+\Lambda_{\beta}$ or the associated field strength $2 \partial_{[\alpha} \mathbb{A}_{r \beta]}$. We can partially fix the symmetries (5.26) to choose a basis $\sigma^{\alpha}=\left(\tau, \sigma^{i}\right)$ and explicitly set $\beta^{\alpha}=\beta_{0} \delta_{\tau}^{\alpha}$ and $\Lambda_{\beta}=\beta_{0} \mu_{0}$, where $\beta_{0}=\left(k_{\mathrm{B}} T_{0}\right)^{-1}$ is the constant inverse temperature and $\mu_{0}$ the chemical potential of the global thermal state. The residual symmetry transformations are then the arbitrary spatial relabelling of fluid elements $\sigma^{i} \rightarrow \sigma^{\prime i}(\vec{\sigma})$, spatial redefinitions of the local time coordinate $\tau \rightarrow \tau+f(\vec{\sigma})$, and that of the $\mathrm{U}(1)$ phase fields $\varphi_{s} \rightarrow \varphi_{s}+\lambda(\vec{\sigma})$. Note that the arbitrary redefinitions are not allowed to depend on $\tau$ itself, because the labelling scheme chosen at one point in time cannot be arbitrarily changed as the fluid evolves. If the fluid has some of its spacetime or U(1) symmetries spontaneously broken, as in superfluids or crystals, the symmetries (5.26) will need to be respectively lifted.

The SK effective action for Galilean hydrodynamics takes the schematic form

$$
S\left[\mathfrak{n}_{1}, \mathfrak{h}_{1}, \mathbb{A}_{1}, \mathfrak{n}_{2}, \mathfrak{h}_{2}, \mathbb{A}_{2} ; \mathbb{B}, \Lambda_{\mathbb{\beta}}\right]=\int \mathrm{d}^{d+1} \sigma \sqrt{\varpi_{r}} \mathcal{L}\left[\mathfrak{n}_{1}, \mathfrak{h}_{1}, \mathbb{A}_{1}, \mathfrak{n}_{2}, \mathfrak{h}_{2}, \mathbb{A}_{2} ; \mathbb{B}, \Lambda_{\mathbb{\beta}}\right] .
$$

Here $\varpi_{r}=\operatorname{det}\left(\mathfrak{n}_{r \alpha} \mathfrak{n}_{r \beta}+\mathfrak{h}_{r \alpha \beta}\right)$ with $\mathfrak{n}_{r \alpha}=\left(\mathfrak{n}_{1 \alpha}+\mathfrak{n}_{1 \alpha}\right) / 2$ and $\mathfrak{h}_{r \alpha \beta}=\left(\mathfrak{h}_{1 \alpha \beta}+\mathfrak{h}_{1 \alpha \beta}\right) / 2$. The Lagrangian $\mathcal{L}$ is a gauge and Milne-invariant scalar on the worldvolume with appropriate contraction of $\alpha, \beta, \ldots$ indices. The Milne invariance is hard to implement within the NC language. In practise, it is much easier to start from the null background effective action (3.5) and identify

$$
\begin{aligned}
\mathfrak{I}_{s \mathrm{AB}}(\sigma) \mathrm{d} \sigma^{\mathrm{A}} \mathrm{d} \sigma^{\mathrm{B}} & =-2\left(\mathrm{~d} \sigma^{-}-\mathbb{A}_{s \beta}(\sigma) \mathrm{d} \sigma^{\beta}\right) \mathfrak{n}_{s \alpha}(\sigma) \mathrm{d} \sigma^{\alpha}+\mathfrak{h}_{s \alpha \beta}(\sigma) \mathrm{d} \sigma^{\alpha} \mathrm{d} \sigma^{\beta}, \\
\mathbb{V}^{\mathrm{A}}(\sigma) \partial_{\mathrm{A}} & =\partial_{-}, \quad \mathbb{\beta}^{\mathrm{A}}(\sigma) \partial_{\mathrm{A}}=\beta^{\alpha}(\sigma) \partial_{\alpha}-\Lambda_{\beta}(\sigma) \partial_{-} .
\end{aligned}
$$

We can define the SK double copies of Galilean currents by varying the effective action 
with respect to various sources (according to eq. (3.6)), leading to

$$
\begin{aligned}
\rho_{1}^{\mu}\left(X_{1}\right) & =\frac{\hbar}{\sqrt{\gamma_{1}\left(X_{1}\right)} \frac{\delta S}{\delta A_{1 \mu}\left(X_{1}\right)},} & \rho_{2}^{\mu}\left(X_{2}\right) & =\frac{-\hbar}{\sqrt{\gamma_{2}\left(X_{2}\right)}} \frac{\delta S}{\delta A_{2 \mu}\left(X_{2}\right)}, \\
\epsilon_{1}^{\mu}\left(X_{1}\right) & =\frac{-\hbar}{\sqrt{\gamma_{1}\left(X_{1}\right)} \frac{\delta S}{\delta n_{1 \nu}\left(X_{1}\right)},} & \epsilon_{2}^{\mu}\left(X_{2}\right) & =\frac{\hbar}{\sqrt{\gamma_{2}\left(X_{2}\right)}} \frac{\delta S}{\delta n_{2 \nu}\left(X_{2}\right)}, \\
\tau_{1}^{\mu \nu}\left(X_{1}\right) & =\frac{2 \hbar h_{1 \rho}^{\nu}\left(X_{1}\right) h_{1 \sigma}^{\mu}\left(X_{1}\right)}{\sqrt{\gamma_{1}\left(X_{1}\right)}} \frac{\delta S}{\delta h_{1 \rho \sigma}\left(X_{1}\right)}, & \tau_{2}^{\mu \nu}\left(X_{2}\right) & =\frac{-2 \hbar h_{2 \rho}^{\nu}\left(X_{2}\right) h_{2 \sigma}^{\mu}\left(X_{2}\right)}{\sqrt{\gamma_{2}\left(X_{2}\right)}} \frac{\delta S}{\delta h_{2 \rho \sigma}\left(X_{2}\right)},
\end{aligned}
$$

with $\gamma_{s}=\operatorname{det}\left(n_{s \mu} n_{s \nu}+h_{s \mu \nu}\right)$. The relative signs between the two copies of the operators arises from the respective second copies being inserted on the time-reversed part of the SK contour. We have taken $S$ to be unitless, leading to the additional factors of $\hbar$ in these formulae. Classical equations of motion for the dynamical fields $X_{s}^{\mu}$ and $\varphi_{s}$ imply that these operators satisfy the conservation equations (5.9) independently on the two SK spacetimes.

\subsubsection{Effective field theory on physical spacetime}

We can translate the above fluid worldvolume framework into a more useful physical spacetime language. The idea is that we can use the average coordinates $x^{\mu}=X_{r}^{\mu}(\sigma)$ as coordinates on the physical NC spacetime. Inverting this map, we can express the spacetime labels as dynamical fields on the physical spacetime $\sigma^{\alpha}=\sigma^{\alpha}(x)$. Accordingly, pushforward the remaining dynamical fields leads to the physical $\mathrm{U}(1)$ phase $\varphi_{r}(x)=\varphi_{r}(\sigma(x))$ and the stochastic noise fields $X_{a}^{\mu}(x)=X_{a}^{\mu}(\sigma(x))$ and $\varphi_{a}(x)=\varphi_{a}(\sigma(x))$.

Let us decompose the fluid worldvolume invariants (5.24) into average and difference combinations $\mathfrak{n}_{1,2 \alpha}=\mathfrak{n}_{r \alpha} \pm \hbar / 2 \mathfrak{n}_{a \alpha}, \mathfrak{h}_{1,2 \alpha \beta}=\mathfrak{h}_{r \alpha \beta} \pm \hbar / 2 \mathfrak{h}_{a \alpha \beta}$, and $\mathbb{A}_{1,2 \alpha}=\mathbb{A}_{r \alpha} \pm \hbar / 2 \mathbb{A}_{a \alpha}$. It should be noted that while $\mathbb{h}_{1,2 \alpha \beta}$ are degenerate, their linear combinations $\mathbb{h}_{a \alpha \beta}$ are not generically degenerate. We can define the pullbacks onto the physical spacetime using $\sigma^{\alpha}(x)$ and $\varphi_{r}(x)$ (similar to eq. (3.8)) as $^{24}$

$$
\begin{aligned}
N_{r \mu}(x) & =\mathfrak{\natural}_{r \alpha}(\sigma(x)) \partial_{\mu} \sigma^{\alpha}(x)=n_{r \mu}(x)+\mathcal{O}(\hbar), \\
H_{r \mu \nu}(x) & =\mathfrak{h}_{r \alpha \beta}(\sigma(x)) \partial_{\mu} \sigma^{\alpha}(x) \partial_{\nu} \sigma^{\beta}(x)=h_{r \mu \nu}(x)+\mathcal{O}(\hbar), \\
B_{r \mu}(x) & =\mathbb{A}_{r \alpha}(\sigma(x)) \partial_{\mu} \sigma^{\alpha}(x)-\partial_{\mu} \varphi_{r}(x)=A_{r \mu}(x)+\mathcal{O}(\hbar), \\
N_{a \mu}(x) & =\mathfrak{\complement}_{a \alpha}(\sigma(x)) \partial_{\mu} \sigma^{\alpha}(x)=n_{a \mu}(x)+£_{X_{a}} n_{r \mu}(x)+\mathcal{O}(\hbar), \\
H_{a \mu \nu}(x) & =\mathfrak{h}_{a \alpha \beta}(\sigma(x)) \partial_{\mu} \sigma^{\alpha}(x) \partial_{\nu} \sigma^{\beta}(x)=h_{a \mu \nu}(x)+£_{X_{a}} h_{r \mu \nu}+\mathcal{O}(\hbar), \\
B_{a \mu}(x) & =\mathbb{A}_{a \alpha}(\sigma(x)) \partial_{\mu} \sigma^{\alpha}(x)=A_{a \mu}(x)+\partial_{\mu} \varphi_{a}(x)+£_{X_{a}} A_{r \mu}(x)+\mathcal{O}(\hbar) .
\end{aligned}
$$

Here $£_{X_{a}}$ denotes a Lie derivative along $X_{a}^{\mu}(x)$. These quantities are invariant under the fluid worldvolume symmetries (5.26), however they transform quite non-trivially under Milne boosts (5.25). Note that $\mathbb{A}_{r \alpha}$ is the only worldvolume quantity that transforms under the fluid worldvolume gauge transformations and hence is subjected to a gauge shift during

\footnotetext{
${ }^{24}$ Please don't confuse the clock-form pullbacks $N_{r, a \mu}$ with the classical free energy current $N^{\mu}$.
} 
the pullback. ${ }^{25}$ In the second equalities, we have decomposed the respective background fields into average and difference combinations according to $n_{1,2 \mu}=n_{r \mu} \pm \hbar / 2 n_{a \mu}, h_{1,2 \mu \nu}=$ $h_{r \mu \nu} \pm \hbar / 2 h_{a \mu \nu}$, and $A_{1,2 \mu}=A_{r \mu} \pm \hbar / 2 A_{a \mu}$, and expanded the expressions up to leading order in $\hbar .^{26}$ We can identify the average background fields with the classical single-copy NC background fields introduced in section 5.1. Since the SK background frame velocities $v_{1,2}^{\mu}(x)$ are in our hand, we can always choose these to be parallel to each other, i.e.

$$
v_{1,2}^{\mu}(x)=\frac{v^{\mu}(x)}{v^{\mu}(x) n_{1,2 \mu}(x)}, \quad v^{\mu}(x) \equiv \frac{v_{1}^{\mu}(x)}{v_{1}^{\nu}(x) n_{r \mu}(x)}=\frac{v_{2}^{\mu}(x)}{v_{2}^{\nu}(x) n_{r \mu}(x)} .
$$

Note that $v^{\mu} n_{r \mu}=1$. This ensures that $h_{r, a \mu \nu}$ are degenerate and satisfy $v^{\mu} h_{r, a \mu \nu}=0$, and fixes the "off-diagonal" part of the Milne redundancy. Note that the objects $H_{r, a \mu \nu}$ for finite $\hbar$ are still not guaranteed to be degenerate. The average inverse spatial metric $h_{r}^{\mu \nu}$ can be defined in the usual manner via $h_{r}^{\mu \nu} n_{r \nu}=0$ and $h_{r}^{\mu \lambda} h_{r \lambda \nu}+v^{\mu} n_{r \nu}=\delta_{\nu}^{\mu} .{ }^{27}$

We can similarly obtain the "hydrodynamic fields" $\beta^{\mu}(x)$ and $\Lambda_{\beta}(x)\left(=-\beta^{-}(x)\right)$ by pushforward of $\beta^{\alpha}(\sigma)$ and $\Lambda_{\beta}(\sigma)$ respectively (according to eq. (3.10))

$$
\beta^{\mu}(x)=\beta^{\alpha}(\sigma(x)) \partial_{\alpha} X_{r}^{\mu}(\sigma(x)), \quad \Lambda_{\beta}(x)=\Lambda_{\beta}(\sigma(x))+\beta^{\alpha}(\sigma(x)) \partial_{\alpha} \varphi_{r}(\sigma(x)) .
$$

If we were to choose $\beta^{\alpha}(\sigma)=\beta_{0} \delta_{\tau}^{\alpha}$ and $\Lambda_{\beta}=\beta_{0} \mu_{0}$, these definitions merely become $\beta^{\mu}=$ $\beta_{0} \partial_{\tau} X_{r}^{\mu}$ and $\Lambda_{\beta}=\beta_{0}\left(\mu_{0}+\partial_{\tau} \varphi_{r}\right)$. The conventional Newton-Cartan hydrodynamic fields: fluid velocity $u^{\mu}(x)$ (normalised as $u^{\mu} N_{r \mu}=1$ ), local temperature $T(x)$, and mass chemical potential $\mu(x)$ are defined as (see eq. (3.11))

$$
\begin{aligned}
k_{\mathrm{B}} T(x) & =\frac{1}{\beta^{\mu}(x) N_{r \mu}(x)}, \quad u^{\mu}(x)=\frac{\beta^{\mu}(x)}{\beta^{\lambda}(x) N_{r \lambda}(x)}, \\
\mu(x) & =\frac{\beta^{\mu}(x) B_{r \mu}(x)+\Lambda_{\beta}(x)}{\beta^{\lambda}(x) N_{r \lambda}(x)}+\frac{1}{2} \frac{\beta^{\mu}(x) \beta^{\nu}(x)}{\left(\beta^{\lambda}(x) N_{r \lambda}(x)\right)^{2}} H_{r \mu \nu}(x)+\frac{\hbar^{2}}{4} \frac{\beta^{\mu}(x) \beta^{\nu}(x) N_{a \mu} B_{a \nu}}{\left(\beta^{\lambda}(x) N_{r \lambda}(x)\right)^{2}} .
\end{aligned}
$$

Note that in the statistical limit $(\hbar \rightarrow 0)$, all the dependence on the non-stochastic dynamical fields $\sigma^{\alpha}(x)$ and $\varphi_{r}(x)$ in the physical spacetime formulation comes only via the hydrodynamic fields. This justifies the validity of the choice of degrees of freedom in classical hydrodynamics.

In the physical spacetime representation, all the fluid worldvolume symmetries of the effective theory are explicitly realised. As a payoff, however, we need to implement the "average" 28 part of the SK spacetime symmetries (5.23) translated onto the physical spacetime

\footnotetext{
${ }^{25}$ During the relativistic discussion in [21] the authors chose to not to apply a gauge shift in the definition of $B_{r \mu}$. This does not make any practical difference except that the resultant $B_{r \mu}$ would transform under the fluid worldvolume gauge transformations and invariant under the physical spacetime ones. Also, in the statistical limit, it behaves as $B_{r \mu} \rightarrow A_{r \mu}+\partial_{\mu} \varphi_{r}+\mathcal{O}(\hbar)$.

${ }^{26}$ See footnote 13.

${ }^{27}$ The " $1 / 2 "$ inverse spatial metrics are non-trivially related as $h_{1,2}^{\mu \nu}=h_{r}^{\mu \nu} \mp \hbar / 2 h_{r}^{\mu \rho} h_{r}^{\nu \sigma} h_{a \rho \sigma} \mp$ $\hbar v^{(\mu} h_{r}^{\nu) \rho} n_{a \rho}+\mathcal{O}\left(\hbar^{2}\right)$. Similarly $h_{1,2}{ }_{\nu}{ }_{\nu}=h_{r \nu}^{\mu}-\hbar / 2 v^{\mu} h_{r \nu}^{\lambda} n_{a \lambda}+\mathcal{O}\left(\hbar^{2}\right)$.

${ }^{28}$ Explicitly in terms of eq. (5.23), one finds

$$
\begin{aligned}
x^{\prime \mu}(x) & =\frac{1}{2}\left(X_{1}^{\prime \mu}\left(x+\hbar / 2 X_{a}(x)\right)+X_{2}^{\prime \mu}\left(x-\hbar / 2 X_{a}(x)\right)\right)=\frac{1}{2}\left(X_{1}^{\prime \mu}(x)+X_{2}^{\prime \mu}(x)\right)+\mathcal{O}(\hbar), \\
\Lambda(x) & =\frac{1}{2}\left(\Lambda_{1}\left(x+\hbar / 2 X_{a}(x)\right)+\Lambda_{2}\left(x-\hbar / 2 X_{a}(x)\right)\right)=\frac{1}{2}\left(\Lambda_{1}(x)+\Lambda_{2}(x)\right)+\mathcal{O}(\hbar) .
\end{aligned}
$$
}


through the maps $\sigma(x)$ and $\varphi_{r}(x)$, i.e.

$$
x^{\mu} \rightarrow x^{\prime \mu}(x), \quad \varphi_{r}(x) \rightarrow \varphi_{r}(x)-\Lambda(x) .
$$

Various fields transform under these as usual

$$
\begin{aligned}
N_{r \mu}(x) \rightarrow N_{r \mu}^{\prime}\left(x^{\prime}\right) & =\frac{\partial x^{\nu}}{\partial x^{\prime \mu}} N_{r \nu}(x), & N_{a \mu \nu}(x) \rightarrow N_{a \mu \nu}^{\prime}\left(x^{\prime}\right) & =\frac{\partial x^{\nu}}{\partial x^{\prime \mu}} N_{a \nu}(x), \\
H_{r \mu \nu}(x) \rightarrow H_{r \mu \nu}^{\prime}\left(x^{\prime}\right) & =\frac{\partial x^{\rho}}{\partial x^{\prime \mu}} \frac{\partial x^{\sigma}}{\partial x^{\prime \nu}} H_{r \rho \sigma}(x), & H_{a \mu \nu}(x) \rightarrow H_{a \mu \nu}^{\prime}\left(x^{\prime}\right) & =\frac{\partial x^{\rho}}{\partial x^{\prime \mu}} \frac{\partial x^{\sigma}}{\partial x^{\prime \nu}} H_{a \rho \sigma}(x), \\
B_{r \mu}(x) \rightarrow B_{r \mu}^{\prime}\left(x^{\prime}\right) & =\frac{\partial x^{\nu}}{\partial x^{\prime \mu}}\left(B_{r \nu}(x)+\partial_{\nu} \Lambda(x)\right), & B_{a \mu}(x) \rightarrow B_{a \mu}^{\prime}\left(x^{\prime}\right) & =\frac{\partial x^{\nu}}{\partial x^{\prime \mu}} B_{a \nu}(x), \\
\beta^{\mu}(x) & \rightarrow \beta^{\prime \mu}\left(x^{\prime}\right)=\frac{\partial x^{\prime \mu}(\sigma)}{\partial x^{\nu}} \mathbb{\beta}^{\nu}(x), & \Lambda_{\beta}(x) \rightarrow \Lambda_{\beta}^{\prime}\left(x^{\prime}\right) & =\Lambda_{\beta}(x)-\beta^{\mu}(x) \partial_{\mu} \Lambda(x) .
\end{aligned}
$$

Note that the chemical potential $\mu(x)$ defined through eq. (5.33) is gauge invariant.

The hydrodynamic effective action (5.27) can also be translated to the physical spacetime language leading to

$$
S\left[N_{r}, H_{r}, B_{r}, N_{a}, H_{a}, B_{a} ; \beta, \Lambda_{\beta}\right]=\int \mathrm{d}^{d+1} x \sqrt{\gamma_{r}} \mathcal{L}\left[N_{r}, H_{r}, B_{r}, N_{a}, H_{a}, B_{a} ; \beta, \Lambda_{\beta}\right],
$$

with $\gamma_{r}=\operatorname{det}\left(n_{r \mu} n_{r \nu}+h_{r \mu \nu}\right)$. The action is manifestly invariant under all the worldvolume and physical spacetime symmetries, with the exception of Milne invariance. The latter can be implemented in practise by starting from the null background effective action (3.13) with the identification

$$
\begin{aligned}
G_{r \mathrm{MN}}(x) \mathrm{d} x^{\mathrm{M}} \mathrm{d} x^{\mathrm{N}}= & -2\left(\mathrm{~d} x^{-}-B_{r \nu}(x) \mathrm{d} x^{\nu}\right) N_{r \mu}(x) \mathrm{d} x^{\mu} \\
& +\left(H_{r \mu \nu}(x)+\frac{\hbar^{2}}{2} N_{a(\mu}(x) B_{a \nu}(x)\right) \mathrm{d} x^{\mu} \mathrm{d} x^{\nu}, \\
G_{a \mathrm{MN}}(x) \mathrm{d} x^{\mathrm{M}} \mathrm{d} x^{\mathrm{N}}= & -2\left(\mathrm{~d} x^{-}-B_{r \nu}(x) \mathrm{d} x^{\nu}\right) N_{a \mu}(x) \mathrm{d} x^{\mu}+\left(H_{a \mu \nu}(x)+2 N_{r(\mu}(x) B_{a \nu)}(x)\right) \mathrm{d} x^{\mu} \mathrm{d} x^{\nu}, \\
V^{\mathrm{M}}(x) \partial_{\mathrm{M}}= & \partial_{-}, \quad \beta^{\mathrm{M}}(x) \partial_{\mathrm{M}}=\beta^{\mu}(x) \partial_{\mu}-\Lambda_{\beta}(x) \partial_{-} .
\end{aligned}
$$

We can use eq. (5.29) to define " $r / a$ " basis of various Galilean observables. We obtain the physical mass current, energy current, and stress tensor by varying with respect to " $a$ " sources

$$
\begin{aligned}
\rho_{r}^{\mu}(x) & =\frac{1 / \hbar}{\sqrt{\gamma_{r}(x)}} \frac{\delta S}{\delta A_{a \mu}(x)}=\frac{1 / 2}{\sqrt{\gamma_{r}(x)}}\left(\sqrt{\gamma_{1}(x)} \rho_{1}^{\mu}(x)+\sqrt{\gamma_{2}(x)} \rho_{2}^{\mu}(x)\right), \\
\epsilon_{r}^{\mu}(x) & =\frac{-1 / \hbar}{\sqrt{\gamma_{r}(x)}} \frac{\delta S}{\delta n_{a \mu}(x)}=\frac{1 / 2}{\sqrt{\gamma_{r}(x)}}\left(\sqrt{\gamma_{1}(x)} \epsilon_{1}^{\mu}(x)+\sqrt{\gamma_{2}(x)} \epsilon_{2}^{\mu}(x)\right), \\
\tau_{r}^{\mu \nu}(x) & =\frac{2 / \hbar h_{r \rho}^{\mu} h_{r \sigma}^{\nu}}{\sqrt{\gamma_{r}(x)}} \frac{\delta S}{\delta h_{a \rho \sigma}(x)}=\frac{1 / 2 h_{r \rho}^{\mu} h_{r \sigma}^{\nu}}{\sqrt{\gamma_{r}(x)}}\left(\sqrt{\gamma_{1}(x)} \tau_{1}^{\rho \sigma}(x)+\sqrt{\gamma_{2}(x)} \tau_{2}^{\rho \sigma}(x)\right)+\mathcal{O}(\hbar) .
\end{aligned}
$$

In the statistical limit, they turn into the average combinations of the SK spacetime transformations. 
while the associated stochastic noise is obtained by varying with respect to " $r$ " sources

$$
\begin{aligned}
\rho_{a}^{\mu}(x) & =\frac{1 / \hbar}{\sqrt{\gamma_{r}(x)}} \frac{\delta S}{\delta A_{r \mu}(x)}=\frac{1 / \hbar}{\sqrt{\gamma_{r}(x)}}\left(\sqrt{\gamma_{1}(x)} \rho_{1}^{\mu}(x)-\sqrt{\gamma_{2}(x)} \rho_{2}^{\mu}(x)\right), \\
\epsilon_{a}^{\mu}(x) & =\frac{-1 / \hbar}{\sqrt{\gamma_{r}(x)}} \frac{\delta S}{\delta n_{r \mu}(x)}=\frac{1 / \hbar}{\sqrt{\gamma_{r}(x)}}\left(\sqrt{\gamma_{1}(x)} \epsilon_{1}^{\mu}(x)-\sqrt{\gamma_{2}(x)} \epsilon_{2}^{\mu}(x)\right), \\
\tau_{a}^{\mu \nu}(x) & =\frac{2 / \hbar h_{r \rho}^{\mu} h_{r \sigma}^{\nu}}{\sqrt{\gamma_{r}(x)}} \frac{\delta S}{\delta h_{r \rho \sigma}(x)}=\frac{1 / \hbar h_{r \rho}^{\mu} h_{r \sigma}^{\nu}}{\sqrt{\gamma_{r}(x)}}\left(\sqrt{\gamma_{1}(x)} \tau_{1}^{\rho \sigma}(x)-\sqrt{\gamma_{2}(x)} \tau_{2}^{\rho \sigma}(x)\right)+\mathcal{O}(\hbar) .
\end{aligned}
$$

These can also be derived directly via null reduction of eq. (3.14). While both set of quantities satisfy conservation equations in flat spacetime, they are not individually conserved in the presence of background sources. The physical operators are, however, conserved in small $\hbar$ limit. Note that we have only simplified expressions for stress tensor $\tau_{r}^{\mu \nu}$ and respective noise $\tau_{a}^{\mu \nu}$ within in small $\hbar$ expansion; expressions for finite $\hbar$ are quite involved.

\subsubsection{Schwinger-Keldysh constraints and explicit effective action}

The SK generating functional for Galilean hydrodynamics can be defined same as eq. (3.15) in the worldvolume formulation or eq. (3.16) in the physical spacetime formulation. Outof-equilibrium ordered correlations functions (retarded, advanced, symmetric etc.) can be obtained by varying the generating functional with respect to " $r / a$ " combinations of background sources similar to eq. (3.17). The generating functional must obey the SK constraints given in eq. (3.19), with the map between the NC and higher-dimensional sources given in eq. (5.1). Within the effective field theory, these constraints are implemented by requiring the effective action to obey eq. (3.21) in the worldvolume formulation or eq. (3.25) in the physical spacetime formulation; note the maps eq. (5.28) and eq. (5.36) between the $\mathrm{NC}$ and null background ingredients.

For reference, we note the KMS conjugation properties of various NC objects. These are similar to the ones proposed for the relativistic case in [12]. Firstly, the background sources transform as

$$
\begin{aligned}
\tilde{n}_{1 \mu}(x) & =\Theta n_{1 \mu}(x), & \tilde{n}_{2 \mu}(x) & =\Theta n_{2 \mu}\left(t+i \hbar \beta_{0}, \vec{x}\right), \\
\tilde{h}_{1 \mu \nu}(x) & =\Theta h_{1 \mu \nu}(x), & \tilde{h}_{2 \mu \nu}(x) & =\Theta h_{2 \mu \nu}\left(t+i \hbar \beta_{0}, \vec{x}\right), \\
\tilde{A}_{1 \mu}(x) & =\Theta A_{1 \mu}(x), & \tilde{A}_{2 \mu}(x) & =\Theta A_{2 \mu}\left(t+i \hbar \beta_{0}, \vec{x}\right) .
\end{aligned}
$$

This leads to their average and difference combinations transforming in the statistical limit as

$$
\begin{aligned}
& \tilde{n}_{r \mu}(x)=\Theta n_{r \mu}(x)+\mathcal{O}(\hbar), \quad \tilde{n}_{a \mu}(x)=\Theta n_{a \mu}(x)+i \beta_{0} \Theta \partial_{t} n_{r \mu}(x)+\mathcal{O}(\hbar), \\
& \tilde{h}_{r \mu \nu}(x)=\Theta h_{r \mu \nu}(x)+\mathcal{O}(\hbar), \quad \tilde{h}_{a \mu \nu}(x)=\Theta h_{a \mu \nu}(x)+i \beta_{0} \Theta \partial_{t} h_{r \mu \nu}(x)+\mathcal{O}(\hbar), \\
& \tilde{A}_{r \mu}(x)=\Theta A_{r \mu}(x)+\mathcal{O}(\hbar), \quad \tilde{A}_{a \mu \nu}(x)=\Theta A_{a \mu}(x)+i \beta_{0} \Theta \partial_{t} A_{r \mu}(x)+\mathcal{O}(\hbar) .
\end{aligned}
$$


For the fluid worldvolume quantities we find ${ }^{29}$

$$
\begin{aligned}
& \tilde{X}_{1}^{\mu}(\sigma)=\Theta X_{1}^{\mu}(\sigma), \quad \tilde{X}_{2}^{\mu}(\sigma)=\Theta X_{2}^{\mu}(\sigma+i \hbar \Theta \beta(\sigma))-i \hbar \beta_{0} \delta_{t}^{\mu}, \\
& \tilde{\varphi}_{1}(\sigma)=\Theta \varphi_{1}(\sigma), \quad \tilde{\varphi}_{2}(\sigma)=\Theta \varphi_{2}(\sigma+i \hbar \Theta \beta(\sigma))+i \hbar \Theta \Lambda_{\beta}(\sigma), \\
& \tilde{\beta}^{\alpha}(\sigma)=\Theta \beta^{\alpha}(\sigma), \quad \tilde{\Lambda}_{\beta}(\sigma)=\Theta \Lambda_{\beta}(\sigma), \\
& \tilde{\mathrm{n}}_{1 \alpha}(\sigma)=\Theta \mathfrak{h}_{1 \alpha}(\sigma), \quad \tilde{\mathrm{m}}_{2 \alpha}(\sigma)=\partial_{\alpha}\left(\sigma^{\beta}+i \hbar \Theta \beta^{\beta}(\sigma)\right) \Theta \mathrm{n}_{2 \beta}(\sigma+i \hbar \Theta \beta(\sigma)), \\
& \tilde{\mathfrak{h}}_{1 \alpha \beta}(\sigma)=\Theta \mathfrak{h}_{1 \alpha \beta}(\sigma), \quad \tilde{\mathfrak{h}}_{2 \alpha \beta}(\sigma)=\partial_{\alpha}\left(\sigma^{\gamma}+i \hbar \Theta \beta^{\gamma}(\sigma)\right) \partial_{\beta}\left(\sigma^{\delta}+i \hbar \Theta \beta^{\delta}(\sigma)\right) \Theta \mathfrak{h}_{2 \gamma \delta}(\sigma+i \hbar \Theta \beta(\sigma)) \text {, } \\
& \tilde{\mathbb{A}}_{1 \alpha}(\sigma)=\Theta \mathbb{A}_{1 \alpha}(\sigma), \quad \tilde{\mathbb{A}}_{2 \alpha}(\sigma)=\partial_{\alpha}\left(\sigma^{\beta}+i \hbar \Theta \beta^{\beta}(\sigma)\right) \Theta \mathbb{A}_{2 \beta}(\sigma+i \hbar \Theta \beta(\sigma))-i \hbar \Theta \partial_{\alpha} \Lambda_{\beta}(\sigma) .
\end{aligned}
$$

The argument $(\sigma+i \hbar \Theta \beta(\sigma))$ should be understood as a vector, i.e. $\sigma^{\alpha}+i \hbar \Theta \beta^{\alpha}(\sigma)$. The KMS conjugation in the "1" sector is merely a $\Theta$-conjugation, while in the "2" sector it is given by a $\Theta$-conjugation followed by a diffeomorphism along $i \hbar \Theta \beta^{\alpha}(\sigma)$ and a gauge shift along $i \hbar \Theta \Lambda_{\beta}(\sigma)$. On the physical spacetime we find (in the statistical limit)

$$
\begin{aligned}
\tilde{\sigma}^{\alpha}(x) & =\Theta \sigma^{\alpha}(x), & \tilde{X}_{a}^{\mu}(x) & =\Theta X_{a}^{\mu}(x)-i \Theta \beta^{\mu}(x)+i \beta_{0} \delta_{t}^{\mu}+\mathcal{O}(\hbar), \\
\tilde{\varphi}_{r}(x) & =\Theta \varphi_{r}(x), & \tilde{\varphi}_{a}(x) & =\Theta \varphi_{a}(x)-i \Theta \Lambda_{\beta}(x)+\mathcal{O}(\hbar), \\
\tilde{\beta}^{\mu}(x) & =\Theta \beta^{\mu}(x)+\mathcal{O}(\hbar), & \tilde{\Lambda}_{\beta}(x) & =\Theta \Lambda_{\beta}(x)+\mathcal{O}(\hbar), \\
\tilde{N}_{r \mu}(x) & =\Theta N_{r \mu}(x)+\mathcal{O}(\hbar), & \tilde{N}_{a \mu}(x) & =\Theta N_{a \mu}(x)+i \Theta \delta_{\mathscr{B}} N_{r \mu}(x)+\mathcal{O}(\hbar), \\
\tilde{H}_{r \mu \nu}(x) & =\Theta H_{r \mu \nu}(x)+\mathcal{O}(\hbar), & \tilde{H}_{a \mu \nu}(x) & =\Theta H_{a \mu \nu}(x)+i \Theta \delta_{\mathscr{B}} H_{r \mu \nu}(x)+\mathcal{O}(\hbar), \\
\tilde{B}_{r \mu}(x) & =\Theta B_{r \mu}(x)+\mathcal{O}(\hbar), & \tilde{B}_{a \mu \nu}(x) & =\Theta B_{a \mu}(x)+i \Theta \delta_{\mathscr{B}} B_{r \mu}(x)+\mathcal{O}(\hbar) .
\end{aligned}
$$

The operator $\delta_{\mathscr{B}}$ combines a Lie derivative along $\beta^{\mu}$ and a gauge transformation along $\Lambda_{\beta}$, i.e. $\delta_{\mathscr{B}} N_{r \mu}=£_{\beta} N_{r \mu}, \delta_{\mathscr{B}} H_{r \mu \nu}=£_{\beta} H_{r \mu \nu}$, and $\delta_{\mathscr{B}} B_{r \mu}=£_{\beta} B_{r \mu}+\partial_{\mu} \Lambda_{\beta}$.

In the statistical limit, the KMS transformation acts on the building blocks of the effective action simply as $\mathscr{B} \rightarrow \Theta \mathscr{B}, \Phi_{r} \rightarrow \Theta \Phi_{r}$, and $\Phi_{a} \rightarrow \Theta \Phi_{a}+i \Theta \delta_{\mathscr{B}} \Phi_{r}$ for the hydrodynamic fields $\mathscr{B}=\left(\beta^{\mu}, \Lambda_{\beta}\right)$ and the invariants $\Phi_{r, a}=\left(N_{r, a \mu}, 1 / 2 H_{r, a \mu \nu}, B_{r, a \mu}\right)$. The construction of the explicit effective action allowed by the KMS constraints follows similar to section 3.4 with a trivial substitution of $1 / 2 G_{r, a} \rightarrow \Phi_{r, a}$ and $£_{\beta} \rightarrow \delta_{\mathscr{B}}$. In particular, the most general effective for Galilean hydrodynamics is given in terms of a set of real totally-symmetric multi-linear operators $\mathcal{D}_{m}(\circ, \ldots)$ made out of $\Phi_{r}$ and $\mathscr{B}$, allowing $m$ number of arguments

\footnotetext{
${ }^{29}$ If we were to fix $\beta^{\alpha}(\sigma)=\beta_{0} \delta_{\tau}^{\alpha}$ and $\Lambda_{\mathbb{\beta}}(\sigma)=\beta_{0} \mu_{0}$, the transformations in the "2" sector will merely become

$$
\tilde{X}_{2}^{\mu}(\sigma)=\Theta X_{2}^{\mu}\left(\tau+i \hbar \beta_{0}, \vec{\sigma}\right)-i \hbar \beta_{0} \delta_{t}^{\mu}, \quad \tilde{\varphi}_{2}(\sigma)=\Theta \varphi_{2}\left(\tau+i \hbar \beta_{0}, \vec{\sigma}\right)+i \hbar \beta_{0} \mu_{0}
$$$$
\tilde{\mathrm{n}}_{2 \alpha}(\sigma)=\Theta \mathrm{n}_{2 \alpha}\left(\tau+i \hbar \beta_{0}, \vec{\sigma}\right), \quad \tilde{\mathfrak{h}}_{2 \alpha \beta}(\sigma)=\Theta \mathfrak{h}_{2 \alpha \beta}\left(\tau+i \hbar \beta_{0}, \vec{\sigma}\right), \quad \tilde{\mathbb{A}}_{2 \alpha}(\sigma)=\Theta \mathbb{A}_{2 \alpha}\left(\tau+i \hbar \beta_{0}, \vec{\sigma}\right),
$$

similar to the ones proposed in [21] (up to a constant shift of phase). 
from the vector space spanned by $i \delta_{\mathscr{B}} \Phi_{r}$ and $\Phi_{a}$; to wit

$$
\begin{aligned}
\mathcal{L}= & \mathcal{D}_{1}\left(\Phi_{a}\right)+i \sum_{n=1}^{\infty} \mathcal{D}_{2 n}(\underbrace{\Phi_{a}, \ldots}_{\times n}, \underbrace{\Phi_{a}+i \delta_{\mathscr{B}} \Phi_{r}, \ldots}_{\times n}) \\
& +\sum_{n=1}^{\infty} \mathcal{D}_{2 n+1}(\Phi_{a}+\frac{i}{2} \delta_{\mathscr{B}} \Phi_{r}, \underbrace{\Phi_{a}, \ldots}_{\times n}, \underbrace{\Phi_{a}+i \delta_{\mathscr{B}} \Phi_{r}, \ldots}_{\times n})+\mathcal{O}(\hbar) .
\end{aligned}
$$

The operators satisfy three constraints

$$
\begin{aligned}
& \mathcal{D}_{1}\left(\delta_{\mathscr{B}} \Phi_{r}\right)=\frac{1}{\sqrt{\gamma_{r}}} \partial_{\mu}\left(\sqrt{\gamma_{r}} \mathcal{N}_{0}^{\mu}\right) \text { for some vector } \mathcal{N}_{0}^{\mu}, \\
& \mathcal{D}_{m}\left(\Phi_{a}, \Phi_{a}, \ldots\right) \text { is } \Theta \text {-even } \forall m, \\
& \left.\mathcal{D}_{2}(\Phi, \Phi)\right|_{\text {leading order }} \geq 0 \text { for arbitrary } \Phi=\left(N_{\mu}, 1 / 2 H_{\mu \nu}, B_{\mu}\right) .
\end{aligned}
$$

The classical constitutive relations only get contributions from the first three operators $\mathcal{D}_{1,2,3}$. These agree with the adiabaticity equation (5.20), which leads to the emergent local second law of thermodynamics. The proof of the same follows from our discussion in section 3.4.

For example, the effective action for one-derivative Galilean hydrodynamics can be reduced from its null fluid incarnation in (4.3). We find a slightly cumbersome expression

$$
\begin{aligned}
\mathcal{L}= & \rho u^{\mu}\left(A_{a \mu}+\partial_{\mu} \varphi_{a}+£_{X_{a}} A_{r \mu}\right) \\
- & \left(\left(\varepsilon+p+\frac{1}{2} \rho h_{r \rho \sigma} u^{\rho} u^{\sigma}\right) u^{\mu}-p v^{\mu}\right)\left(n_{a \mu}+£_{X_{a}} n_{r \mu}\right)+\frac{1}{2}\left(\rho u^{\mu} u^{\nu}+p h_{r}^{\mu \nu}\right)\left(h_{a \mu \nu}+£_{X_{a}} h_{r \mu \nu}\right) \\
+ & \left(-\frac{a_{0}}{2} \epsilon^{\mu \nu \rho \sigma} n_{\nu} \Omega_{\rho \sigma}+\frac{a_{2}}{2} \epsilon^{\mu \nu \rho \sigma} n_{\nu} F_{r \rho \sigma}^{n}\right) \\
& \left(\left(A_{a \mu}+\partial_{\mu} \varphi_{a}+£_{X_{a}} A_{r \mu}\right)+u^{\lambda}\left(h_{a \lambda \mu}+£_{X_{a}} h_{r \lambda \mu}\right)-\left(\vec{u}_{\mu} u^{\lambda}+\frac{1}{2} \vec{u}^{2} \delta_{\mu}^{\lambda}\right)\left(n_{a \lambda}+£_{X_{a}} n_{r \lambda}\right)\right) \\
- & \left(-\frac{a_{2}}{2} \epsilon^{\mu \nu \rho \sigma} n_{\nu} \Omega_{\rho \sigma}+\frac{a_{1}}{2} \epsilon^{\mu \nu \rho \sigma} n_{\nu} F_{r \rho \sigma}^{n}\right)\left(n_{a \mu}+£_{X_{a}} n_{\mu}\right) \\
+ & \frac{i k_{\mathrm{B}} T}{4}\left(2 \eta h_{r}^{\mu(\rho} h_{r}^{\sigma) \nu}+\left(\zeta-\frac{2}{d} \eta\right) h_{r}^{\mu \nu} h_{r}^{\rho \sigma}\right)\left(h_{a \mu \nu}+£_{X_{a}} h_{r \mu \nu}-2\left(n_{a(\mu}+£_{X_{a}} n_{r(\mu}\right) h_{r \nu) \lambda} u^{\lambda}\right) \\
& \quad\left(h_{a \rho \sigma}+£_{\left(X_{a}+i \beta\right)} h_{r \rho \sigma}-2\left(n_{a(\mu}+£_{\left(X_{a}+i \beta\right)} n_{r(\mu}\right) h_{r \nu) \lambda} u^{\lambda}\right) \\
+ & i k_{\mathrm{B}} T^{2} \kappa h_{r}^{\mu \nu}\left(n_{a \mu}+£_{X_{a}} n_{r \mu}\right)\left(n_{a \nu}+£_{\left(X_{a}+i \beta\right)} n_{r \nu}\right) \\
& \left(A_{a \mu}+\partial_{\mu} \varphi_{a}+£_{X_{a}} A_{r \mu}\right)-\epsilon^{\mu}\left(n_{a \mu}+£_{X_{a}} n_{r \mu}\right)+\left(v^{\mu} \pi^{\nu}+\frac{1}{2} \tau^{\mu \nu}\right)\left(h_{a \mu \nu}+£_{X_{a}} h_{r \mu \nu}\right) \\
+ & \frac{i k_{\mathrm{B}} T}{4}\left(2 \eta h_{r}^{\mu(\rho} h_{r}^{\sigma) \nu}+\left(\zeta-\frac{2}{d} \eta\right) h_{r}^{\mu \nu} h_{r}^{\rho \sigma}\right)\left(h_{a \mu \nu}+£_{X_{a}} h_{r \mu \nu}-2\left(n_{a(\mu}+£_{X_{a}} n_{r(\mu}\right) h_{r \nu) \lambda} u^{\lambda}\right) \\
& \quad\left(h_{a \rho \sigma}+£_{X_{a}} h_{r \rho \sigma}-2\left(n_{a(\mu}+£_{X_{a}} n_{r(\mu}\right) h_{r \nu) \lambda} u^{\lambda}\right) \\
+ & i k_{\mathrm{B}} T^{2} \kappa h_{r}^{\mu \nu}\left(n_{a \mu}+£_{X_{a}} n_{r \mu}\right)\left(n_{a \nu}+£_{X_{a}} n_{r \nu}\right) .
\end{aligned}
$$

Here $£_{X_{a}}$ denotes a Lie derivative along $X_{a}^{\mu}$ and $£_{\left(X_{a}+i \beta\right)}$ along $X_{a}^{\mu}+i \beta^{\mu}$. In the second step, we have substituted the thermodynamic mass frame constitutive relations from 
eq. (5.19). This is the generalisation of the effective action (1.2) presented in the introduction to include the parity-violating terms. It can be explicitly checked that varying the action with respect to difference sources $A_{a \mu}, n_{a \mu}$, and $h_{a \mu \nu}$, in a configuration with zero " $a$ " type fields $X_{a}^{\mu}=\varphi_{a}=A_{a \mu}=n_{a \mu}=h_{a \mu \nu}$, leads to the classical constitutive relations eq. (5.19), while extremising with respect to $X_{a}^{\mu}$ and $\varphi_{a}$ leads to the conservation equations (5.9).

\section{Outlook}

In this work, we constructed a Schwinger-Keldysh effective field theory for dissipative Galilean (non-relativistic) hydrodynamics. We used the null background formalism of Galilean field theories to recast Galilean fluids in $(d+1)$ spacetime dimensions in terms of relativistic null fluids in $(d+2)$ dimensions. This allowed us to write down an EFT for Galilean fluids along the lines of its relativistic cousin developed recently [12], with appropriate modifications to account for a null Killing vector. We explicitly constructed the most generic effective action allowed by the formalism in the statistical limit, where the quantum fluctuations are suppressed compared to thermal fluctuations. We also inspected how the field theory gives rise to an emergent second law of thermodynamics in the classical limit. As a concrete example, we looked at the EFT for one-derivative dissipative Galilean fluids. We also looked at the linearised limit of this theory, truncated to three point interactions, which is appropriate to describe stochastic fluctuations in a Galilean fluid around its equilibrium state.

The $(d+2)$-dimensional null fluid formalism is also convenient as it makes the Galilean (Milne) boost symmetry manifest within the EFT, however the presence of an auxiliary coordinate direction does obscure the underlying physics to some degree. Therefore, we also spent some time translating our results to the more widely used $(d+1)$-dimensional NewtonCartan formulation of Galilean field theories. While the two formulations are entirely equivalent at the end of the day, the latter is technically more challenging as it involves a non-invertible spatial metric, a torsional connection, and requires one to introduce a Galilean observer to distinguish between energy and momentum. In the NC language, the underlying structure of the EFT looks quite similar to that of charged relativistic hydrodynamics from [12], but with relativistic symmetries and background sources swapped for the Galilean ones. The Galilean EFT can also be arrived at by taking a non-relativistic limit of the relativistic theory, as we illustrate inappendix B.

Several generalisations of this work are immediately possible. We have assumed the Galilean symmetry group underlying the fluid to be unbroken, but the EFT requirements can also be relaxed to describe fluid phases with spontaneously broken symmetries. For example, in the null background language, to describe Galilean superfluids with spontaneously broken $\mathrm{U}(1)$ we can provide each fluid element with a reference phase $\phi(\sigma)$. We must restrict the derivatives of the phase as $\beta^{\mathrm{A}} \partial_{\mathrm{A}} \phi=0$ (since the reference phase must be fixed in the comoving frame of the fluid elements) and $\mathbb{V}^{\mathrm{A}} \partial_{\mathrm{A}} \phi=1$ (required for spontaneously breaking the $\mathrm{U}(1)) .{ }^{30}$ On the physical spacetime, this results in the reference phase

\footnotetext{
${ }^{30}$ Choosing $\beta^{\mathrm{A}}=\beta_{0}\left(\delta_{\tau}^{\mathrm{A}}-\mu_{0} \delta_{-}^{\mathrm{A}}\right)$ and $\mathbb{V}^{\mathrm{A}}=\delta_{-}^{\mathrm{A}}$, these conditions merely say that $\partial_{-} \phi=1$ and $\partial_{\tau} \phi=\mu_{0}$. Recall
} 
satisfying $\beta^{\mathrm{M}} \partial_{\mathrm{M}} \phi=0$ and $V^{\mathrm{M}} \partial_{\mathrm{M}} \phi=1$, leading to the Josephson's equation for Galilean superfluids $u^{\mathrm{M}} \partial_{\mathrm{M}} \phi=\mu$. In the NC language, the Josephson's equation is equivalently stated as $u^{\mu} \xi_{\mu}=\mu-\frac{1}{2} \vec{u}^{2}$, where $\xi_{\mu}=\partial_{\mu} \phi+A_{r \mu}$ is identified as the superfluid velocity; see [55, 57] for more discussion in case of classical Galilean superfluids. Similarly, we can construct an EFT for viscoelastic hydrodynamics with spontaneously broken translations by including reference "crystal coordinate fields" $\phi^{I}(\sigma)$ with $I=1, \ldots, d$ satisfying $\beta^{\mathrm{A}} \partial_{\mathrm{A}} \phi^{I}=0$ and $\mathbb{V}^{\mathrm{A}} \partial_{\mathrm{A}} \phi^{I}=0$; see $[69,70]$ for a classical analogue in the relativistic case. More complicated symmetry breaking patterns are also possible, describing a various phases of liquid crystals. The reference fields to be included for such phases can be inferred by employing a coset construction similar to [25].

The EFT can also be extended to the cases where the underlying global symmetries are anomalous. In the simplest case that we are considering, the U(1) mass symmetry does not admit any anomalies [53]. The system can admit gravitational/rotational anomalies, but these only show up in $d=4 n+1$ spatial dimensions at $(4 n+2)$ th derivative order in the parity-violating sector. In particular, there are no such anomalies in $d=3$ and none at one-derivative order in general. If one were to include additional symmetries in the Galilean hydrodynamic setup, such as additional U(1) or non-Abelian charges (e.g. electromagnetic or flavor), the anomaly structure can be much more non-trivial; see [33, 53]. The inclusion of anomalies into the EFT, in such cases, would follow similar to the relativistic discussion in e.g. [19]. Generalisations are also possible to more exotic theories of hydrodynamics with higher-form symmetries (see e.g. [71-75]). So far, such dissipative EFTs have not been constructed even in the relativistic case; see [26] for a non-dissipative discussion of relativistic hydrodynamics with one-form symmetries.

In a more pragmatic direction, one can use the EFT for stochastic fluctuations from section 4.2 to compute stochastic loop corrections to classical hydrodynamic correlation functions. A similar analysis was done for the simplified case of energy diffusion (with no momentum or density modes) in [23]. Authors wrote down the most generic effective action describing energy diffusion at one-derivative order and quartic order in fluctuations, and worked out one-loop stochastic corrections to energy-energy two-point functions. The discussion in section 4.2 can, in principle, be extended to include quartic and higher interactions as well. However, as far as one-loop corrections to two-point functions are concerned, even in full hydrodynamics, the quartic interactions only contribute to renormalise the hydrodynamic parameters (shear and energy diffusion constants, susceptibility, etc.) and do not lead to any cutoff independent predictions.

Formally, the EFT for hydrodynamics has been setup for finite $\hbar$, capable of describing both stochastic and quantum fluctuations within the same effective framework. However, to concretely implement the KMS condition, we were forced to work in the small $\hbar$ (statistical)

that choosing this basis fixed the worldvolume diffeomorphisms (3.4) down to spatial $\sigma^{i}$ diffeomorphisms, and residual spatial reparametrisations of $\sigma^{\tau}$ and $\sigma^{-}$. We can further fix the $\sigma^{-}$-reparametrisations explicitly by choosing $\phi(\sigma)=\sigma^{-}+\mu_{0} \sigma^{\tau}$. In the NC language, this amounts to lifting the worldvolume phase shift symmetry in eq. (5.26). This is similar to what was proposed for relativistic superfluids in [21]. One must be careful in the Galilean case, however, because although $\mathbb{A}_{r \sigma}$ is now gauge invariant, it still transforms under Milne boosts. 
limit. It is still an open question, whether or not the effective KMS symmetry can be implemented at finite $\hbar$. We should note that, in this work, we have focused on the hydrodynamic EFT framework of [12]. There is another contending framework due to [20], where the discrete KMS symmetry is replaced by a local $U(1)_{T}$ symmetry; see [76] for a comparison. It will be interesting to revisit our Galilean results in the context [20].

\section{Acknowledgments}

The author would like to thank Jay Armas, Pavel Kovtun, Kristan Jensen, and Hong Liu for various helpful discussions and comments. This work is supported in part by the NSERC Discovery Grant program of Canada.

\section{A Second law constraints in Galilean hydrodynamics}

In this appendix we give a derivation of the second law constraints in Galilean hydrodynamics. For ease, we will work in the null fluid framework. Translation to NC language is straight-forward. We start with the decomposition of null fluid energy-momentum tensor as in eq. (2.25) and write down expressions for $p^{\mathrm{MN}}$ and $q^{\mathrm{M}}$ satisfying $p^{\mathrm{MN}} u_{\mathrm{N}}=p^{\mathrm{MN}} V_{\mathrm{N}}=0$ and $q^{\mathrm{M}} u_{\mathrm{M}}=q^{\mathrm{M}} V_{\mathrm{M}}=0$

$$
\begin{aligned}
p^{\mathrm{MN}} & =p \Delta^{\mathrm{MN}}-\eta \sigma^{\mathrm{MN}}-\zeta \Delta^{\mathrm{MN}} \nabla_{\mathrm{R}} u^{\mathrm{R}}, \\
q^{\mathrm{M}} & =-\kappa \Delta^{\mathrm{MN}} \nabla_{\mathrm{N}} T+T \tilde{\kappa} 2 \nabla^{[\mathrm{M}} V^{\mathrm{N}]} u_{\mathrm{N}}+\xi_{\Omega} \epsilon^{\mathrm{MNRST}} V_{\mathrm{N}} u_{\mathrm{R}} \nabla_{\mathrm{S}} u_{\mathrm{T}}+\xi_{H} \epsilon^{\mathrm{MNRST}} V_{\mathrm{N}} u_{\mathrm{R}} \nabla_{\mathrm{S}} V_{\mathrm{T}} .
\end{aligned}
$$

At this point, all the coefficients appearing above are completely arbitrary. We have introduced two new coefficients $\tilde{\kappa}$ and $\xi_{H}$ coupled to $2 \nabla_{[\mathrm{M}} V_{\mathrm{N}]}=2 \partial_{[\mathrm{M}} g_{\mathrm{N}]-}$ which do not contribute on a flat background. These are similar to terms coupled to gauge field strength $F_{\mu \nu}$ in relativistic theories. Similarly, for the non-canonical part of the entropy current in eq. (2.25) we write down

$$
\Upsilon^{\mathrm{M}}=\frac{a_{2}}{T} \epsilon^{\mathrm{MNRST}} V_{\mathrm{N}} u_{\mathrm{R}} \nabla_{\mathrm{S}} u_{\mathrm{T}}+\frac{a_{1}}{2 T} \epsilon^{\mathrm{MNRST}} V_{\mathrm{N}} u_{\mathrm{R}} \nabla_{\mathrm{S}} V_{\mathrm{T}}-\frac{a_{0}}{2 T} \epsilon^{-\mathrm{MNRS}} u_{\mathrm{N}} \nabla_{\mathrm{R}} u_{\mathrm{S}}
$$

The final term does not transform nicely under symmetries, but the respective contribution to entropy current divergence can be made to behave nicely with a judicious choice of the coefficient $a_{0}$. We have only written down parity-violating terms in $\Upsilon^{\mathrm{M}}$, because divergence of any one-derivative parity-preserving term always contains a term with a doublederivative acting on a quantity, which cannot be made positive semi-definite. Computing 
the entropy-current divergence, we find

$$
\begin{aligned}
\nabla_{\mathrm{M}} S^{\mathrm{M}}= & \frac{\eta}{2 T} \sigma^{\mathrm{MN}} \sigma_{\mathrm{MN}}+\frac{\zeta}{T}\left(\nabla_{\mathrm{M}} u^{\mathrm{M}}\right)^{2}+\kappa \Delta^{\mathrm{MN}}\left(\frac{1}{T} \nabla_{\mathrm{M}} T-2 \nabla_{[\mathrm{M}} V_{\mathrm{R}]} u^{\mathrm{R}}\right)\left(\frac{1}{T} \nabla_{\mathrm{N}} T-2 \nabla_{[\mathrm{N}} V_{\mathrm{S}]} u^{\mathrm{S}}\right) \\
& -(\tilde{\kappa}-\kappa) H^{\mathrm{MR}} u_{\mathrm{R}}\left(\frac{1}{T} \nabla_{\mathrm{M}} T-2 \nabla_{[\mathrm{M}} V_{\mathrm{S}]} u^{\mathrm{S}}\right) \\
& -\frac{1}{T}\left(\xi_{\Omega}-a_{2}+a_{0} \frac{\varepsilon+p}{\rho}\right) \epsilon^{\mathrm{MNRST}} V_{\mathrm{N}} u_{\mathrm{R}} \nabla_{\mathrm{S}} u_{\mathrm{T}}\left(\frac{1}{T} \nabla_{\mathrm{M}} T-2 \nabla_{[\mathrm{M}} V_{\mathrm{P}]} u^{\mathrm{P}}\right) \\
& -\frac{1}{T}\left(\xi_{H}-a_{1}+a_{2} \frac{\epsilon+p}{\rho}\right) \epsilon^{\mathrm{MNRST}} V_{\mathrm{N}} u_{\mathrm{R}} \nabla_{\mathrm{S}} V_{\mathrm{T}}\left(\frac{1}{T} \nabla_{\mathrm{M}} T-2 \nabla_{[\mathrm{M}} V_{\mathrm{P}]} u^{\mathrm{P}}\right) \\
& +T \nabla_{\mathrm{M}}\left(\frac{a_{2}-a_{0} \mu}{T^{2}}\right) \epsilon^{\mathrm{MNRST}} V_{\mathrm{N}} u_{\mathrm{R}} \nabla_{\mathrm{S}} u_{\mathrm{T}}+\frac{T^{2}}{2}\left(\nabla_{\mathrm{M}} \frac{a_{1}}{T^{3}}-\frac{2 a_{2}}{T^{2}} \nabla_{\mathrm{M}} \frac{\mu}{T}\right) \epsilon^{\mathrm{MNRST}} V_{\mathrm{N}} u_{\mathrm{R}} \nabla_{\mathrm{S}} V_{\mathrm{T}} \\
& -\nabla_{\mathrm{M}}\left(\frac{a_{0}}{2 T}\right) \epsilon^{-\mathrm{MNRS}} u_{\mathrm{N}} \nabla_{\mathrm{R}} u_{\mathrm{S}}+\mathcal{O}\left(\partial^{3}\right) .
\end{aligned}
$$

In deriving this expression, we used the first order equations of motion. Terms in the first line can be made positive semi-definite by requiring

$$
\eta \geq 0, \quad \zeta \geq 0, \quad \kappa \geq 0 .
$$

All the remaining terms, unfortunately, must be required to vanish. This leads to equality constraints

$$
\begin{aligned}
& \tilde{\kappa}=\kappa \\
& a_{0}=2 K_{0} T, \quad a_{1}=2 K_{1} T^{3}+2 K_{2} T^{2} \mu+2 K_{0} T \mu^{2}, \quad a_{2}=K_{2} T^{2}+2 K_{0} T \mu, \\
& \xi_{\Omega}=a_{2}-\frac{\varepsilon+p}{\rho} a_{0}, \quad \xi_{H}=a_{1}-\frac{\epsilon+p}{\rho} a_{2},
\end{aligned}
$$

for some constants $K_{0}, K_{1}, K_{2}$. Note that the constant $K_{1}$ only appears in $\lambda_{H}$ and $a_{1}$ and does not contribute in the absence of sources.

\section{B Effective field theory via non-relativistic limit}

In this section we outline a systematic procedure to perform the non-relativistic limit of relativistic hydrodynamics, to arrive at the Galilean results presented in the main text. This essentially entails judiciously introducing factors of $c$ (speed of light) so as to segregate spatial and temporal components of various fields, and take $c \rightarrow \infty$ in the relevant equations.

\section{B.1 Relativistic hydrodynamics}

\section{B.1.1 Classical hydrodynamics}

Let us start with a lightening recap of relativistic hydrodynamics. Classical hydrodynamics is formulated in terms of conserved currents. However, unlike the Galilean case discussed in section 2 , the conserved currents for charged relativistic hydrodynamics are the covariant energy-momentum tensor $T^{\mu \nu}$ and charge/particle number current $J^{\mu}$, associated with Poincaré and $\mathrm{U}(1)$ invariance of the underlying microscopic theory respectively. The indices $\mu, \nu, \ldots$ run over the $(d+1)$-dimensional spacetime coordinates. Coupling the setup to a 
background metric $g_{\mu \nu}$ with signature $(-1,1,1, \ldots)$ and gauge field $A_{\mu}$, the conservation equations are expressed as

$$
\nabla_{\mu} T^{\mu \nu}=F^{\nu \rho} J_{\rho}, \quad \nabla_{\mu} J^{\mu}=0 .
$$

Here $\nabla_{\mu}$ is the covariant derivative associated with $g_{\mu \nu}$ and $F_{\mu \nu}=2 \partial_{[\mu} A_{\nu]}$ is the antisymmetric field strength tensor. We pick an appropriate set of degrees of freedom, called the hydrodynamic fields, to be solved for using the conservation equations: fluid velocity $u^{\mu}$ normalised as $u^{\mu} u_{\mu}=-c^{2}$, temperature $T$, and chemical potential $\mu$. We leave the factors of speed of light $c$ explicit to make contact with Galilean physics later. Normally, one needs to pick a hydrodynamic frame to fix the inherent redefinition freedom in these variables; for now we leave this freedom unfixed.

Hydrodynamic constitutive relations are the most generic expressions for $T^{\mu \nu}$ and $J^{\mu}$, in accordance with symmetries, written in terms of the dynamical fields $u^{\mu}, T, \mu$, and the background fields $g_{\mu \nu}, A_{\mu}$, arranged in a derivative expansion. These constitutive relations are required to satisfy the local second law of thermodynamics. Similar to our discussion in section 2.4, the second law can be stated offshell in terms of the adiabaticity equation [62], i.e. there must exist a free energy current $N^{\mu}$ and a quadratic form $\Delta$ such that

$$
\nabla_{\mu} N^{\mu}=\frac{1}{2} T^{\mu \nu} \delta_{\mathscr{B}} g_{\mu \nu}+J^{\mu} \delta_{\mathscr{B}} A_{\mu}+\Delta, \quad \Delta \geq 0,
$$

is satisfied offshell. Here $\delta_{\mathscr{B}} g_{\mu \nu}=£_{\beta} g_{\mu \nu}$ and $\delta_{\mathscr{B}} A_{\mu}=£_{\beta} A_{\mu}+\partial_{\mu} \Lambda_{\beta}$ denotes a Lie derivative along $\beta^{\mu}=u^{\mu} /\left(k_{\mathrm{B}} T\right)$ and gauge shift along $\Lambda_{\beta}=\left(\mu-u^{\mu} A_{\mu}\right) /\left(k_{\mathrm{B}} T\right)$. The entropy current is defined as $S^{\mu}=k_{\mathrm{B}} N^{\mu}-\left(\mu J^{\mu}+T^{\mu \nu} u_{\nu}\right) / T$, whose divergence satisfies $\nabla_{\mu} S^{\mu}=k_{\mathrm{B}} \Delta \geq 0$ onshell, leading to the sign definiteness of entropy production. By expressing the adiabaticity equation in the form (B.2), we have partially fixed the redefinition freedom in $u^{\mu}, T$, and $\mu$ noted above, formally known as a "thermodynamic frame". The residual freedom can be fixed by requiring certain tensor structures to be eliminated from the constitutive relations using equations of motion. We will make the choice to eliminate $u^{\mu} \delta_{\mathscr{B}} A_{\mu}$ and $u^{\mu} \delta_{\mathscr{B}} g_{\mu \nu}$, which leads to the "thermodynamic Landau frame" wherein the non-hydrostatic (nhs) part of the constitutive relations are transverse to the fluid velocity $T_{\mathrm{nhs}}^{\mu \nu} u_{\nu}=J_{\mathrm{nhs}}^{\mu} u_{\mu}=0$. This is distinct from the conventional "Landau frame" employed in [61], where the full constitutive relations are chosen to obey $T^{\mu \nu} u_{\nu}=-\varepsilon(T, \mu) u^{\mu}$ and $J^{\mu} u_{\mu}=-n(T, \mu)$, with $\varepsilon$ and $n$ being the thermodynamic energy and charge densities respectively.

For example, truncated to one-derivative order, the constitutive relations of a charged relativistic fluid in thermodynamic Landau frame are given as [64] (see [57] for a review) ${ }^{31}$

$$
\begin{aligned}
T^{\mu \nu}= & \frac{1}{c^{2}}(\varepsilon+p) u^{\mu} u^{\nu}+p g^{\mu \nu}-\eta \sigma^{\mu \nu}-\zeta \Delta^{\mu \nu} \nabla_{\lambda} u^{\lambda}+\frac{\alpha_{1}}{c^{5}} 2 u^{(\mu} \epsilon^{\nu) \rho \sigma \lambda} u_{\rho} \partial_{\sigma} u_{\lambda} \\
& +\frac{\alpha_{2}}{2 c^{3}} 2 u^{(\mu} \epsilon^{\nu) \rho \sigma \lambda} u_{\rho} F_{\sigma \lambda}, \\
J^{\mu}= & n u^{\mu}-\sigma \Delta^{\mu \nu}\left(T \partial_{\nu} \frac{\mu}{T}-F_{\nu \lambda} u^{\lambda}\right)+\frac{\alpha_{0}}{2 c} \epsilon^{\mu \nu \rho \sigma} u_{\nu} F_{\rho \sigma}+\frac{\alpha_{2}}{c^{3}} \epsilon^{\mu \nu \rho \sigma} u_{\nu} \partial_{\rho} u_{\sigma},
\end{aligned}
$$

${ }^{31}$ In the conventional Landau frame, the constitutive relations are equivalently given by

$$
\begin{aligned}
T^{\mu \nu} & =\frac{1}{c^{2}}(\epsilon+p) u^{\mu} u^{\nu}+p g^{\mu \nu}-\eta \sigma^{\mu \nu}-\zeta P^{\mu \nu} \nabla_{\lambda} u^{\lambda}, \\
J^{\mu} & =n u^{\mu}-\frac{\sigma}{c^{4}} P^{\mu \nu}\left(T \partial_{\nu} \frac{\mu}{T}-E_{\nu}\right)+\frac{1}{2 c} \xi_{B} \epsilon^{\mu \nu \rho \sigma} u_{\nu} F_{\rho \sigma}+\frac{1}{c^{3}} \xi_{\Omega} \epsilon^{\mu \nu \rho \sigma} u_{\nu} \partial_{\rho} u_{\sigma} .
\end{aligned}
$$

In this frame, the constitutive relations do not satisfy the adiabaticity equation offshell. 
where $\Delta^{\mu \nu}=g^{\mu \nu}+u^{\mu} u^{\nu} / c^{2}$ is the projector transverse to fluid velocity. The convention for the Levi-Civita tensor is $\epsilon_{0123}=\sqrt{-g}$. Thermodynamic pressure $p(T, \mu)$ is related to energy density $\varepsilon(T, \mu)$, charge/particle number density $n(T, \mu)$, and entropy density $s(T, \mu)$ via the thermodynamic relations $\mathrm{d} p=s \mathrm{~d} T+n \mathrm{~d} \mu$ and $\varepsilon=T s+\mu n-p$. In the parityeven sector, $\eta(T, \mu), \zeta(T, \mu)$, and $\sigma(T, \mu)$ are non-negative dissipative transport coefficients identified as shear viscosity, bulk viscosity, and electric conductivity respectively. $\sigma^{\mu \nu}=2 \Delta^{\mu \rho} \Delta^{\nu \sigma}\left(\nabla_{(\rho} u_{\sigma)}-1 / d \Delta_{\rho \sigma} \nabla_{\lambda} u^{\lambda}\right)$ is the fluid shear tensor. While the parity-even part of the constitutive relations is applicable for any number of dimensions, the parityodd part is dimension specific. The above expressions are valid in $d=3$ spatial dimensions, with the transport coefficients

$$
\alpha_{0}=2 C_{0} T, \quad \alpha_{1}=2 C_{1} T^{3}+2 C_{2} T^{2} \mu+2 C_{0} T \mu^{2}, \quad \alpha_{2}=C_{2} T^{2}+2 C_{0} T \mu,
$$

for three arbitrary constants $C_{0}, C_{1}, C_{2} \cdot{ }^{32}$ These are related to the physical parity-odd conductivities as $\xi_{B}=\alpha_{0}-n /(\varepsilon+p) \alpha_{2}$ and $\xi_{\Omega}=\alpha_{2}-n /(\varepsilon+p) \alpha_{1}$ (see footnote 31). The associated free-energy density and quadratic form are given as

$$
\begin{aligned}
k_{\mathrm{B}} N^{\mu} & =\frac{p}{T} u^{\mu}+\frac{\alpha_{1}}{2 T c^{3}} \epsilon^{\mu \nu \rho \sigma} u_{\nu} \partial_{\rho} u_{\sigma}+\frac{\alpha_{2}}{2 T c} \epsilon^{\mu \nu \rho \sigma} u_{\nu} F_{\rho \sigma}+\frac{c}{2} C_{0} \epsilon^{\mu \nu \rho \sigma} A_{\nu} F_{\rho \sigma}, \\
k_{\mathrm{B}} \Delta & =\frac{\eta}{2 T} \sigma^{\mu \nu} \sigma_{\mu \nu}+\frac{\zeta}{T}\left(\nabla_{\lambda} u^{\lambda}\right)^{2}+\frac{\sigma}{T} P^{\mu \nu}\left(T \partial_{\mu} \frac{\mu}{T}-F_{\mu \lambda} u^{\lambda}\right)\left(T \partial_{\nu} \frac{\mu}{T}-F_{\nu \lambda} u^{\lambda}\right) .
\end{aligned}
$$

Note that there is a gauge-non-invariant term in $N^{\mu}$ coupled to $C_{0}$. This is not an issue because the free-energy current $N^{\mu}$ itself is not a physical observable, only the constitutive relations are.

We can also demand the hydrodynamic theory to obey additional constraints like the discrete time-reversal symmetry $\mathrm{T}$, spacetime parity PT, or the full CPT including a charge conjugation, denoted collectively as $\Theta$; see table 2 . These are slightly subtle as they need to be implemented at the microscopic level and not at the level of constitutive relations; we expect the dissipative effects to explicitly violate any kind of $\Theta$-symmetry. Nonetheless, constitutive relations evaluated on a hydrostatic configuration, characterised by $\delta_{\mathscr{B}} g_{\mu \nu}=$ $\delta_{\mathscr{B}} A_{\mu}=0$ in a thermodynamic frame, must respect $\Theta[64,65]$. In the non-hydrostatic sector, discrete symmetries lead to Onsager's reciprocity relations among hydrodynamic retarded two-point functions; see [4]. For instance, taking $\Theta$ to be $\mathrm{T}$ does not lead to any constraints on the constitutive relations (B.4), while PT forces us to set $C_{0,1,2}=0$. On the other hand, choosing $\Theta$ to be $\mathrm{CPT}$ sets $C_{0,1}=0$ leaving only $C_{2}$ in the parity-violating sector [64]. The effective field theory framework deals with these discrete symmetries more systematically, combining them with the thermal KMS condition.

\section{B.1.2 Schwinger-Keldysh effective field theory}

An effective field theory framework for relativistic hydrodynamics has been proposed in [1214]. We will not delve in the technicalities; a comprehensive review can be found in [21]. We directly note the effective action for one-derivative relativistic hydrodynamics in the

\footnotetext{
${ }^{32}$ If the U(1) symmetry is anomalous, eq. (B.5) will also include contributions from the anomaly coefficient.
} 


\begin{tabular}{|c|ccc|c|c|}
\hline & $\mathrm{C}$ & $\mathrm{P}$ & $\mathrm{T}$ & $\mathrm{PT}$ & $\mathrm{CPT}$ \\
\hline$X^{0}, c t=x^{0}, c \tau=\sigma^{0}$ & + & + & - & - & - \\
$X^{i}, x^{i}, \sigma^{i}$ & + & - & + & - & - \\
$\varphi$ & - & + & - & - & + \\
\hline$u^{i}, \beta^{i}, \beta^{i}$ & + & - & - & + & + \\
$T, \beta^{0}, \beta^{0}$ & + & + & + & + & + \\
$\mu, \Lambda_{\beta}, \Lambda_{\beta}$ & - & + & + & + & - \\
\hline$T^{00}, g_{00}$ & + & + & + & + & + \\
$T^{0 i}, g_{0 i}$ & + & - & - & + & + \\
$T^{i j}, g_{i j}$ & + & + & + & + & + \\
$J^{0}, A_{0}$ & - & + & + & + & - \\
$J^{i}, A_{i}$ & - & - & - & + & - \\
\hline
\end{tabular}

Table 2. Action of parity $(\mathrm{P})$, time-reversal $(\mathrm{T})$, and charge conjugation $(\mathrm{C})$ of various quantities in classical relativistic hydrodynamics and effective field theory. Schwinger-Keldysh double copies of various quantities in the effective theory, " $1 / 2$ " or " $r / a$ ", behave the same as their unlabelled versions.

statistical limit. The ingredients we need to introduce are the (physical spacetime formulation) dynamical fields: fluid spacetime labels $\sigma^{\alpha}(x)$, phase field $\varphi_{r}(x)$, associated stochastic noise fields $X_{a}^{\mu}(x), \varphi_{a}(x)$, two sets of background fields $g_{r \mu \nu}(x)=g_{\mu \nu}(x), A_{r \mu}(x)=A_{\mu}(x)$, $g_{a \mu \nu}(x)$, and $A_{a \mu}(x)$, and reference thermal vector $\beta^{\alpha}(\sigma)=\beta_{0} \delta_{\tau}^{\alpha}$ and chemical shift field $\Lambda_{\beta}(\sigma)=\beta_{0} \mu_{0}$. The hydrodynamic fields $\beta^{\mu}(x), \Lambda_{\beta}(x)$ are related to these as same as eq. (5.32). The effective action is found to be

$$
\begin{aligned}
\mathcal{L}= & \frac{1}{2}\left(\frac{\varepsilon+p}{c^{2}} u^{\mu} u^{\nu}+p \Delta^{\mu \nu}+\frac{\alpha_{1}}{c^{5}} 2 u^{(\mu} \epsilon^{\nu) \rho \sigma \lambda} u_{\rho} \partial_{\sigma} u_{\lambda}+\frac{\alpha_{2}}{2 c^{3}} 2 u^{(\mu} \epsilon^{\nu) \rho \sigma \lambda} u_{\rho} F_{\sigma \lambda}\right) G_{a \mu \nu} \\
& +\left(n u^{\mu}+\frac{\alpha_{0}}{2 c} \epsilon^{\mu \nu \rho \sigma} u_{\nu} F_{\rho \sigma}+\frac{\alpha_{2}}{c^{3}} \epsilon^{\mu \nu \rho \sigma} u_{\nu} \partial_{\rho} u_{\sigma}\right) B_{a \mu} \\
& +\frac{i k_{\mathrm{B}} T}{4}\left(2 \eta \Delta^{\mu(\rho} \Delta^{\sigma) \nu}+\left(\zeta-\frac{d}{2} \eta\right) \Delta^{\mu \nu} \Delta^{\rho \sigma}\right) G_{a \mu \nu}\left(G_{a \rho \sigma}+i \delta_{\mathrm{B}} G_{r \rho \sigma}\right) \\
& +i k_{\mathrm{B}} T \sigma P^{\mu \nu} B_{a \mu}\left(B_{a \mu}+i \delta_{\mathscr{B}} B_{r \mu}\right) .
\end{aligned}
$$

Here $G_{r \mu \nu}=g_{r \mu \nu}, B_{r \mu}=A_{\mu}, G_{a \mu \nu}=g_{a \mu \nu}+£_{X_{a}} g_{\mu \nu}$, and $B_{a \mu}=A_{a \mu}+\partial_{\mu} \varphi_{a}+£_{X_{a}} A_{\mu}$. The operator $\delta_{\mathscr{B}}$ acts same as defined below eq. (B.2). It is easy to see that varying with respect to the sources $g_{a \mu \nu}$ and $A_{a \mu}$, and switching off all " $a$ " type fields, one recovers the classical constitutive relations (B.4). The classical conservation equations, on the other hand, are obtained by varying with respect to $X_{a}^{\mu}$ and $\varphi_{a}$.

We note that the effective action (B.7) conforms with the general structure of the effective action advertised in eq. (3.29) with $1 / 2 G_{a}$ replaced with $\Phi_{r, a}=\left(1 / 2 G_{r, a \mu \nu}, B_{r, a \mu}\right)$ and $£_{\beta}$ with $\delta_{\mathscr{B}}$. In fact, with these trivial substitutions, the entire discussion of eq. (B.7) follows through to relativistic hydrodynamics line by line and can be used to extend the effective field theory to arbitrarily high orders in the derivative expansion and noise expansion. 


\section{B.2 Non-relativistic limit}

We are now ready to perform the non-relativistic limit. A general discussion on the procedure can be found in [32]. The limit proceeds in three steps: (1) identify the dynamical and background fields between Galilean hydrodynamics and the relativistic parent, (2) choose $c$-scaling for various transport coefficients, and (3) take $c \rightarrow \infty$. For the first step, we take the prescription from [32]. The second step is hit-and-trial; choosing the $c$-scaling for a transport coefficient to be too high will lead to a divergent $c \rightarrow \infty$ limit and is not good, while choosing it to be too low will cause it to vanish in $c \rightarrow \infty$ limit and we will miss out on some transport coefficients.

Briefly in this section, we use the label "rel" for relativistic quantities in case of notational tension.

\section{B.2.1 Newton-Cartan sources via non-relativistic limit}

A non-relativistic background is characterised by the presence of a preferred one-form $n_{\mu}$ characterising the non-relativistic notion of absolute time. It is convenient to introduce a local Galilean frame velocity $v^{\mu}$ such that $v^{\mu} n_{\mu}=1$. We can use this to decompose the background fields in a series expansion in $c^{2}$ according to

$$
\begin{aligned}
& g_{\mu \nu}=-c^{2} n_{\mu} n_{\nu}+h_{\mu \nu}+\mathcal{O}\left(1 / c^{2}\right), \quad g^{\mu \nu}=-\frac{1}{c^{2}} v^{\mu} v^{\nu}+h^{\mu \nu}+\mathcal{O}\left(1 / c^{2}\right), \\
& A_{\mu}^{\mathrm{rel}}=m c^{2} n_{\mu}+m A_{\mu}+\mathcal{O}\left(1 / c^{2}\right),
\end{aligned}
$$

where $h_{\mu \nu} v^{\nu}=h^{\mu \nu} n_{\nu}=0$ and $n_{\mu} v^{\nu}+h_{\mu \lambda} h^{\lambda \nu}=\delta_{\mu}^{\nu}$. Here $m$ is a constant representing mass per particle. The constituent fields above can be identified with the Newton-Cartan structure discussed in section 5. The relativistic diffeomorphism and U(1) invariance maps equivalently to the Galilean ones given in eq. (5.4). On the other hand, the ambiguity in the choice of the frame velocity $v^{\mu}$ leads to a redundancy, for some $\psi^{\mu}$ satisfying $\psi^{\mu} n_{\mu}=0$,

$$
\begin{array}{lll}
v^{\mu} \rightarrow v^{\mu}+\psi^{\mu}, & h_{\mu \nu} \rightarrow h_{\mu \nu}-2 n_{(\mu} \psi_{\nu)}+n_{\mu} n_{\nu} \psi^{2}, & A_{\mu} \rightarrow A_{\mu}+\psi_{\mu}-\frac{1}{2} n_{\mu} \psi^{2}, \\
n_{\mu} \rightarrow n_{\mu}-\frac{1}{c^{2}}\left(\psi_{\mu}-\frac{1}{2} n_{\mu} \psi^{2}\right), & h^{\mu \nu} \rightarrow h^{\mu \nu}+\frac{1}{c^{2}}\left(2 v^{(\mu} \psi^{\nu)}+\psi^{\mu} \psi^{\nu}\right), & \text { (B.9) }
\end{array}
$$

identified as Galilean Milne invariance in $c \rightarrow \infty$ limit. Since the metric has a $c^{2}$ piece, the associated Levi-Civita connection $\Gamma_{\mu \nu}^{\lambda}$ is not well defined in $c \rightarrow \infty$ limit. Nonetheless, we can define a torsional connection $\tilde{\Gamma}_{\mu \nu}^{\lambda}$ using $g_{\mu \nu}$ and $F_{\mu \nu}^{\text {rel }}$ which behaves regularly. We have

$$
\begin{aligned}
\tilde{\Gamma}_{\mu \nu}^{\lambda} & =\Gamma_{\mu \nu}^{\lambda}-\frac{1}{2 m} g^{\lambda \rho}\left(n_{\rho} F_{\mu \nu}^{\mathrm{rel}}-2 n_{(\mu} F_{\nu) \rho}^{\mathrm{rel}}\right) \\
& =v^{\lambda} \partial_{\mu} n_{\nu}+\frac{1}{2} h^{\lambda \rho}\left(2 \partial_{(\mu} h_{\nu) \rho}-\partial_{\rho} h_{\mu \nu}\right)+n_{(\mu} F_{\nu) \rho} h^{\rho \lambda}+\mathcal{O}(1 / c) .
\end{aligned}
$$

Note that the connection still preserves the relativistic metric $g_{\mu \nu}$. This yields the NewtonCartan connection (5.5) in $c \rightarrow \infty$ limit. Finally, the Levi-Civita tensor scales as $\epsilon_{\mathrm{rel}}^{\mu \nu \rho \sigma}=$ $1 / c \epsilon^{\mu \nu \rho \sigma}$, with the $1 / c$ factor coming from the measure. 
We can define the non-relativistic conserved currents as

$$
\rho^{\mu}=\lim _{c \rightarrow \infty} m J^{\mu}, \quad \epsilon^{\mu}=-\lim _{c \rightarrow \infty}\left(T^{\mu}{ }_{\nu} v^{\nu}+m c^{2} J^{\mu}\right), \quad \tau^{\mu \nu}=\lim _{c \rightarrow \infty} h^{\mu}{ }_{\rho} h^{\nu \sigma} T_{\sigma}^{\rho} .
$$

These limits must be well defined for the system to admit a non-relativistic limit. The Galilean conservation equations follow simply from the relativistic ones ${ }^{33}$

$$
\begin{aligned}
& m \nabla_{\mu} J^{\mu}=\left(\tilde{\nabla}_{\mu}+n_{\mu \lambda} v^{\lambda}\right) \rho^{\mu}+\mathcal{O}(1 / c)=0 \\
& -v^{\nu}\left(\nabla_{\mu} T_{\nu}^{\mu}-F_{\nu \lambda}^{\mathrm{rel}} J^{\lambda}+m c^{2} n_{\nu} \nabla_{\mu} J^{\mu}\right) \\
& \quad=\left(\tilde{\nabla}_{\mu}+n_{\mu \lambda} v^{\lambda}\right) \epsilon^{\mu}-v^{\lambda} n_{\lambda \mu} \epsilon^{\mu}+\left(v^{\mu} \pi^{\lambda}+\tau^{\mu \lambda}\right) h_{\lambda \rho} \tilde{\nabla}_{\mu} v^{\rho}+\mathcal{O}(1 / c)=0 \\
& h^{\rho \nu}\left(\nabla_{\mu} T_{\nu}^{\mu}-F_{\nu \lambda}^{\mathrm{rel}} J^{\lambda}\right) \\
& \quad=\left(\tilde{\nabla}_{\mu}+n_{\mu \lambda} v^{\lambda}\right)\left(v^{\mu} \pi^{\rho}+\tau^{\mu \rho}\right)+h^{\rho \lambda} n_{\lambda \mu} \epsilon^{\mu}+\rho^{\mu} \tilde{\nabla}_{\mu} v^{\rho}+\mathcal{O}(1 / c)=0 .
\end{aligned}
$$

These are same as the ones derived in eq. (5.9).

\section{B.2.2 Non-relativistic limit of hydrodynamics}

Normalisation of the fluid velocity $u^{\mu} u^{\nu} g_{\mu \nu}=-c^{2}$ implies that

$$
u^{\mu} n_{\mu}=1+\frac{1}{2 c^{2}} \vec{u}^{2}+\mathcal{O}\left(1 / c^{4}\right), \quad u_{\mu}=-c^{2} n_{\mu}-\frac{1}{2} \vec{u}^{2} n_{\mu}+\vec{u}_{\mu}+\mathcal{O}\left(1 / c^{2}\right),
$$

where $\vec{u}_{\mu}=h_{\mu \nu} u^{\nu}$ and $\vec{u}^{2}=h_{\mu \nu} u^{\mu} u^{\nu}$. Having set this, through a straight-forward calculation we can deduce that the Galilean fluid constitutive relations (5.15b) follow by taking $c \rightarrow \infty$ in the relativistic fluid constitutive relations (B.4) with the mapping (B.11), provided that we choose

$$
\begin{aligned}
& n=\frac{1}{m} \rho, \quad \varepsilon_{\mathrm{rel}}=\rho c^{2}+\varepsilon, \quad \mu_{\mathrm{rel}}=m c^{2}+m \mu, \quad \sigma=\frac{T \kappa}{m^{2} c^{4}}, \\
& C_{0}=\frac{1}{m^{2}} K_{0}, \quad C_{1}=K_{1}, \quad C_{2}=\frac{1}{m} K_{2} .
\end{aligned}
$$

Most of these identifications are expected on physical grounds: the number density is given by mass density divided by $m$, relativistic energy density has a rest mass contribution and so does the relativistic chemical potential. The mapping of electric conductivity to thermal conductivity is slightly unnatural, but it follows from dimensional analysis. Similarly, mapping of $C_{0,1,2}$ to $K_{0,1,2}$ also follows on dimensional grounds. The remaining relativistic coefficients and quantities map to their namesake on the Galilean side.

Similar mapping rules can be applied to the effective field theory. The dynamical fields $\sigma^{\alpha}, \varphi_{r}$, and $X_{a}^{\mu}, \varphi_{a}$ directly map to their respective Galilean versions, while the average

\footnotetext{
${ }^{33}$ In case the $\mathrm{U}(1)$ symmetry in the relativistic theory is anomalous, i.e. $\nabla_{\mu} J^{\mu}=-3 C /\left(4 c^{5}\right) \epsilon_{\mathrm{rel}}^{\mu \nu \rho} F_{\mu \nu}^{\mathrm{rel}} F_{\rho \sigma}^{\mathrm{rel}}$, the anomaly gets washed away in the non-relativistic limit. It only leads to a shift of the non-relativistic energy current $\epsilon^{\mu} \rightarrow \epsilon^{\mu}-2 C m^{3} \epsilon^{\mu \nu \rho \sigma} n_{\nu} \partial_{\rho} n_{\sigma}$.
} 


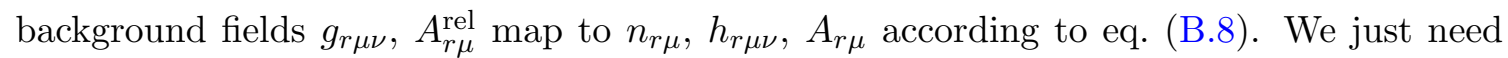
the mapping between the difference background fields, which we propose

$$
g_{a \mu \nu}=-2 c^{2} n_{r(\mu} n_{a \nu)}+h_{a \mu \nu}+\mathcal{O}\left(1 / c^{2}\right), \quad A_{a \mu}^{\mathrm{rel}}=m c^{2} n_{a \mu}+m A_{a \mu}+\mathcal{O}\left(1 / c^{2}\right) .
$$

We leave it to the reader to verify that this identification maps the relativistic one-derivative order effective action (B.7) to the Galilean one in eq. (5.44). In principle, the same procedure can be iterated to arbitrarily high orders in the derivative expansion.

Open Access. This article is distributed under the terms of the Creative Commons Attribution License (CC-BY 4.0), which permits any use, distribution and reproduction in any medium, provided the original author(s) and source are credited.

\section{References}

[1] P.C. Martin, E.D. Siggia and H.A. Rose, Statistical Dynamics of Classical Systems, Phys. Rev. A 8 (1973) 423 [INSPIRE].

[2] Y. Pomeau and P. Resibois, Time Dependent Correlation Functions and Mode-Mode Coupling Theories, Phys. Rept. 19 (1974) 63.

[3] I.M. De Schepper, H. Van Beyeren and M.H. Ernst, The nonexistence of the linear diffusion equation beyond Fick's law, Physica 75 (1974) 1.

[4] P. Kovtun, Lectures on hydrodynamic fluctuations in relativistic theories, J. Phys. A 45 (2012) 473001 [arXiv: 1205. 5040] [INSPIRE].

[5] L. Landau and E. Lifshitz, Statistical Physics, Part 1, vol. 5 of Course of Theoretical Physics, Butterworth-Heinemann, Oxford (1980).

[6] L. Landau and E. Lifshitz, Hydrodynamic fluctuations, Sov. Phys. JETP 5 (1957) 512.

[7] P.C. Hohenberg and B.I. Halperin, Theory of Dynamic Critical Phenomena, Rev. Mod. Phys. 49 (1977) 435 [INSPIRE].

[8] C. De Dominicis and L. Peliti, Field Theory Renormalization and Critical Dynamics Above t(c): Helium, Antiferromagnets and Liquid Gas Systems, Phys. Rev. B 18 (1978) 353 [INSPIRE].

[9] I. Khalatnikov, V. Lebedev and A. Sukhorukov, Diagram technique for calculating long wave fluctuation effects, Phys. Lett. A 94 (1983) 271 [InSPIRE].

[10] S. Dubovsky, L. Hui, A. Nicolis and D.T. Son, Effective field theory for hydrodynamics: thermodynamics, and the derivative expansion, Phys. Rev. D 85 (2012) 085029 [arXiv: 1107.0731] [INSPIRE].

[11] S. Grozdanov and J. Polonyi, Viscosity and dissipative hydrodynamics from effective field theory, Phys. Rev. D 91 (2015) 105031 [arXiv:1305.3670] [InSPIRE].

[12] M. Crossley, P. Glorioso and H. Liu, Effective field theory of dissipative fluids, JHEP 09 (2017) 095 [arXiv: 1511. 03646] [INSPIRE].

[13] P. Glorioso, M. Crossley and H. Liu, Effective field theory of dissipative fluids (II): classical limit, dynamical KMS symmetry and entropy current, JHEP 09 (2017) 096 [arXiv: 1701.07817] [INSPIRE]. 
[14] P. Glorioso and H. Liu, The second law of thermodynamics from symmetry and unitarity, arXiv: 1612.07705 [INSPIRE].

[15] P. Gao and H. Liu, Emergent Supersymmetry in Local Equilibrium Systems, JHEP 01 (2018) 040 [arXiv: 1701.07445 ] [INSPIRE].

[16] P. Glorioso, H. Liu and S. Rajagopal, Global Anomalies, Discrete Symmetries, and Hydrodynamic Effective Actions, JHEP 01 (2019) 043 [arXiv:1710.03768] [INSPIRE].

[17] P. Gao, P. Glorioso and H. Liu, Ghostbusters: Unitarity and Causality of Non-equilibrium Effective Field Theories, JHEP 03 (2020) 040 [arXiv:1803.10778] [INSPIRE].

[18] K. Jensen, N. Pinzani-Fokeeva and A. Yarom, Dissipative hydrodynamics in superspace, JHEP 09 (2018) 127 [arXiv: 1701.07436] [INSPIRE].

[19] K. Jensen, R. Marjieh, N. Pinzani-Fokeeva and A. Yarom, A panoply of Schwinger-Keldysh transport, SciPost Phys. 5 (2018) 053 [arXiv: 1804.04654] [INSPIRE].

[20] F.M. Haehl, R. Loganayagam and M. Rangamani, Effective Action for Relativistic Hydrodynamics: Fluctuations, Dissipation, and Entropy Inflow, JHEP 10 (2018) 194 [arXiv: 1803.11155] [INSPIRE].

[21] H. Liu and P. Glorioso, Lectures on non-equilibrium effective field theories and fluctuating hydrodynamics, PoS TASI2017 (2018) 008 [arXiv:1805.09331] [INSPIRE].

[22] E. Wang and U.W. Heinz, A Generalized fluctuation dissipation theorem for nonlinear response functions, Phys. Rev. D 66 (2002) 025008 [hep-th/9809016] [INSPIRE].

[23] X. Chen-Lin, L.V. Delacrétaz and S.A. Hartnoll, Theory of diffusive fluctuations, Phys. Rev. Lett. 122 (2019) 091602 [arXiv: 1811.12540] [INSPIRE].

[24] P. Glorioso, L.V. Delacrétaz, X. Chen, R.M. Nandkishore and A. Lucas, Hydrodynamics in lattice models with continuous non-Abelian symmetries, arXiv:2007.13753 [INSPIRE].

[25] M.J. Landry, The coset construction for non-equilibrium systems, JHEP 07 (2020) 200 [arXiv: 1912.12301] [INSPIRE].

[26] P. Glorioso and D.T. Son, Effective field theory of magnetohydrodynamics from generalized global symmetries, arXiv: 1811.04879 [INSPIRE].

[27] S.E. Gralla and N. Iqbal, Effective Field Theory of Force-Free Electrodynamics, Phys. Rev. D 99 (2019) 105004 [arXiv: 1811.07438] [INSPIRE].

[28] E. Cartan, Sur les varietes a connexion affine et la theorie de la relativite generalisee (premiere partie) (Suite), Annales Sci. Ecole Norm. Sup. 41 (1924) 1.

[29] E. Cartan, Sur les varietes a connexion affine et la theorie de la relativite generalisee (premiere partie), Annales Sci. Ecole Norm. Sup. 40 (1923) 325.

[30] K. Jensen, On the coupling of Galilean-invariant field theories to curved spacetime, SciPost Phys. 5 (2018) 011 [arXiv: 1408.6855] [INSPIRE].

[31] K. Jensen, Aspects of hot Galilean field theory, JHEP 04 (2015) 123 [arXiv:1411.7024] [INSPIRE].

[32] K. Jensen and A. Karch, Revisiting non-relativistic limits, JHEP 04 (2015) 155 [arXiv: 1412.2738] [INSPIRE].

[33] K. Jensen, Anomalies for Galilean fields, SciPost Phys. 5 (2018) 005 [arXiv:1412.7750] [INSPIRE]. 
[34] G. Festuccia, D. Hansen, J. Hartong and N.A. Obers, Torsional Newton-Cartan Geometry from the Noether Procedure, Phys. Rev. D 94 (2016) 105023 [arXiv:1607.01926] [InSPIRE].

[35] E. Bergshoeff, A. Chatzistavrakidis, L. Romano and J. Rosseel, Newton-Cartan Gravity and Torsion, JHEP 10 (2017) 194 [arXiv: 1708.05414] [INSPIRE].

[36] E.A. Bergshoeff, J. Hartong and J. Rosseel, Torsional Newton-Cartan geometry and the Schrödinger algebra, Class. Quant. Grav. 32 (2015) 135017 [arXiv: 1409.5555] [INSPIRE].

[37] M.H. Christensen, J. Hartong, N.A. Obers and B. Rollier, Boundary Stress-Energy Tensor and Newton-Cartan Geometry in Lifshitz Holography, JHEP 01 (2014) 057 [arXiv: 1311.6471] [INSPIRE].

[38] M.H. Christensen, J. Hartong, N.A. Obers and B. Rollier, Torsional Newton-Cartan Geometry and Lifshitz Holography, Phys. Rev. D 89 (2014) 061901 [arXiv:1311.4794] [INSPIRE].

[39] J. Hartong, N.A. Obers and M. Sanchioni, Lifshitz Hydrodynamics from Lifshitz Black Branes with Linear Momentum, JHEP 10 (2016) 120 [arXiv:1606.09543] [INSPIRE].

[40] J. Hartong, E. Kiritsis and N.A. Obers, Schrödinger Invariance from Lifshitz Isometries in Holography and Field Theory, Phys. Rev. D 92 (2015) 066003 [arXiv:1409.1522] [InSPIRE].

[41] M. Geracie, K. Prabhu and M.M. Roberts, Fields and fluids on curved non-relativistic spacetimes, JHEP 08 (2015) 042 [arXiv:1503.02680] [INSPIRE].

[42] M. Geracie, K. Prabhu and M.M. Roberts, Physical stress, mass, and energy for non-relativistic matter, JHEP 06 (2017) 089 [arXiv:1609.06729] [INSPIRE].

[43] C. Duval and P.A. Horvathy, Non-relativistic conformal symmetries and Newton-Cartan structures, J. Phys. A 42 (2009) 465206 [arXiv:0904.0531] [INSPIRE].

[44] R. Banerjee and P. Mukherjee, Milne boost from galilean gauge theory, Phys. Lett. B 778 (2018) 303 [arXiv: 1710.10882] [INSPIRE].

[45] R. Banerjee and P. Mukherjee, Torsional Newton-Cartan geometry from Galilean gauge theory, Class. Quant. Grav. 33 (2016) 225013 [arXiv: 1604.06893] [INSPIRE].

[46] N. Banerjee, S. Dutta and A. Jain, Null Fluids - A New Viewpoint of Galilean Fluids, Phys. Rev. D 93 (2016) 105020 [arXiv: 1509.04718] [INSPIRE].

[47] J. Maldacena, D. Martelli and Y. Tachikawa, Comments on string theory backgrounds with non-relativistic conformal symmetry, JHEP 10 (2008) 072 [arXiv:0807.1100] [INSPIRE].

[48] A. Adams, K. Balasubramanian and J. McGreevy, Hot Spacetimes for Cold Atoms, JHEP 11 (2008) 059 [arXiv:0807.1111] [INSPIRE].

[49] C.P. Herzog, M. Rangamani and S.F. Ross, Heating up Galilean holography, JHEP 11 (2008) 080 [arXiv: 0807.1099] [INSPIRE].

[50] C. Duval, G. Burdet, H.P. Kunzle and M. Perrin, Bargmann Structures and Newton-cartan Theory, Phys. Rev. D 31 (1985) 1841 [InSPIRE].

[51] C. Duval, G.W. Gibbons and P. Horvathy, Celestial mechanics, conformal structures and gravitational waves, Phys. Rev. D 43 (1991) 3907 [hep-th/0512188] [INSPIRE].

[52] B. Julia and H. Nicolai, Null Killing vector dimensional reduction and Galilean geometrodynamics, Nucl. Phys. B 439 (1995) 291 [hep-th/9412002] [INSPIRE]. 
[53] A. Jain, Galilean Anomalies and Their Effect on Hydrodynamics, Phys. Rev. D 93 (2016) 065007 [arXiv: 1509.05777] [INSPIRE].

[54] N. Banerjee, S. Dutta and A. Jain, Equilibrium partition function for nonrelativistic fluids, Phys. Rev. D 92 (2015) 081701 [arXiv:1505.05677] [INSPIRE].

[55] N. Banerjee, S. Dutta and A. Jain, First Order Galilean Superfluid Dynamics, Phys. Rev. D 96 (2017) 065004 [arXiv: 1612.01550] [INSPIRE].

[56] N. Banerjee, S. Atul Bhatkar and A. Jain, Second order Galilean fluids and Stokes' law, Phys. Rev. D 97 (2018) 096018 [arXiv:1711.09076] [InSPIRE].

[57] A. Jain, A universal framework for hydrodynamics, Ph.D. thesis, Durham University, CPT, $6,2018$.

[58] M. Hassaine and P.A. Horvathy, Field dependent symmetries of a nonrelativistic fluid model, Annals Phys. 282 (2000) 218 [math-ph/9904022] [INSPIRE].

[59] P.A. Horvathy and P.-M. Zhang, Non-relativistic conformal symmetries in fluid mechanics, Eur. Phys. J. C 65 (2010) 607 [arXiv:0906.3594] [InSPIRE].

[60] M.L. Bellac, Thermal Field Theory, Cambridge Monographs on Mathematical Physics 3 (2011) [INSPIRE].

[61] L. Landau and E. Lifshitz, Fluid Mechanics, Teoreticheskaia fizika, Pergamon Press (1959).

[62] R. Loganayagam, Anomaly Induced Transport in Arbitrary Dimensions, arXiv:1106.0277 [INSPIRE].

[63] P. Kovtun, First-order relativistic hydrodynamics is stable, JHEP 10 (2019) 034 [arXiv: 1907.08191] [INSPIRE].

[64] N. Banerjee, J. Bhattacharya, S. Bhattacharyya, S. Jain, S. Minwalla and T. Sharma, Constraints on Fluid Dynamics from Equilibrium Partition Functions, JHEP 09 (2012) 046 [arXiv: 1203.3544] [INSPIRE].

[65] K. Jensen, M. Kaminski, P. Kovtun, R. Meyer, A. Ritz and A. Yarom, Towards hydrodynamics without an entropy current, Phys. Rev. Lett. 109 (2012) 101601 [arXiv: 1203.3556] [INSPIRE].

[66] C. Arzt, Reduced effective Lagrangians, Phys. Lett. B 342 (1995) 189 [hep-ph/9304230] [INSPIRE].

[67] S. Bhattacharyya, Entropy Current from Partition Function: One Example, JHEP 07 (2014) 139 [arXiv: 1403.7639] [INSPIRE].

[68] S. Bhattacharyya, Entropy current and equilibrium partition function in fluid dynamics, JHEP 08 (2014) 165 [arXiv: 1312.0220] [INSPIRE].

[69] J. Armas and A. Jain, Viscoelastic hydrodynamics and holography, JHEP 01 (2020) 126 [arXiv: 1908.01175] [INSPIRE].

[70] J. Armas and A. Jain, Hydrodynamics for charge density waves and their holographic duals, Phys. Rev. D 101 (2020) 121901 [arXiv:2001.07357] [INSPIRE].

[71] S. Grozdanov and N. Poovuttikul, Generalized global symmetries in states with dynamical defects: The case of the transverse sound in field theory and holography, Phys. Rev. D 97 (2018) 106005 [arXiv: 1801.03199] [INSPIRE]. 
[72] S. Grozdanov, D.M. Hofman and N. Iqbal, Generalized global symmetries and dissipative magnetohydrodynamics, Phys. Rev. D 95 (2017) 096003 [arXiv:1610.07392] [INSPIRE].

[73] J. Armas, J. Gath, A. Jain and A.V. Pedersen, Dissipative hydrodynamics with higher-form symmetry, JHEP 05 (2018) 192 [arXiv:1803.00991] [INSPIRE].

[74] J. Armas and A. Jain, One-form superfluids 83 magnetohydrodynamics, JHEP 01 (2020) 041 [arXiv: 1811.04913] [INSPIRE].

[75] J. Armas and A. Jain, Magnetohydrodynamics as superfluidity, Phys. Rev. Lett. 122 (2019) 141603 [arXiv: 1808.01939] [INSPIRE].

[76] F.M. Haehl, R. Loganayagam and M. Rangamani, Two roads to hydrodynamic effective actions: a comparison, arXiv:1701.07896 [INSPIRE]. 